\title{
DISTRIBUTION OF WELLS IN THE CENTRAL PART OF THE WESTERN SAN JOAQUIN VALLEY, CALIFORNIA
}

U.S. GEOLOGICAL SURVEY

Water-Resources Investigations Report 89-4158

REGIONAL AQUIFER SYSTEM ANALYSIS

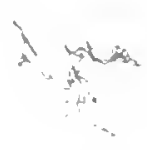


This report was prepared by the U.S. Geological Survey in cooperation with the San Joamuin Valley Drainage Program and as part of the Regional Aquifer-System Analysis (RASA) Program of the U.S. Ceological Survey.

The San Jabquin Valley Dranaye Program was established in mid-1984 and is a cimprative effort of the U.S. Bureau of Reclamation, U.S. Fish and ivildife Serice, II.S. Ceological Survey, Colifornia Department of Fish and Game, and CHifuran Deparment of Hater Resources. The purposes of the program are to invelifute the problems associated with the drainage of argricultural lands in The Sarl Juaquin Valley and to develop solutions to those problems. Consistent with these purposes, proaram objectives address the following key concerns: (3) public health, (2) surface- and ground-water resources, (3) agricultural prodectivity, and (4) fish and wildife resources.

Imquiries concenning the San Joaquin Valley Drainage Program may be directed to:

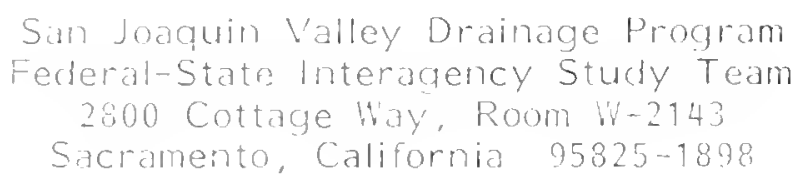

The RASA Program of the U.S. Ceological Survey was started in 1978 following a conmessiona! mandate to develop quantitative appraisals of the major groundwater sustems of the United States. The RASA Program represents a systematic effort to study a number of the Nation's most important aquifer systems, which in Hgaregite underlie much of the country and which represent an important component

the Nation $=$ fotal water supply. In general, the boundaries of these studies 3re Gentified by the hydrologic extent of each system, and accordingly transcend ha bolitical ubdivisions to which investigations were often arbitrarily limited in the pest. The broad objectives for cach stuch are to assemble geologic, hydrologic, a'd Gohemicil information, to analyze and develop an understanding of the sysem, and in develop predictive capabilities that will contribute to the effective mangenent the system. The Central Valley RASA study, which focused on the ...trology and geochemistry of ground water in the Central Valley of Califormia, Legan in: 1979. Phase 11 of the Central Valley RASA began in 1984 and is in prupess. The focus during this second phase is on more detailed study of the and gechemistry of ground water in the San Joaquin Valley, which is the sutherr halt of the Central Valley. 
DISTRIBUTION OF WELLS IN THE CENTRAL PART OF THE

WESTERN SAN JOAQUIN VALLEY, CALIFORNIA

By Jo Ann M. Gronberg, Kenneth Belitz, and Steven P. Phillips

U.S. GEOLOGICAL SURVEY

Water-Resources Investigations Report 89-4158

REGIONAL AQUIFER SYSTEM ANALYSIS

Prepared in cooperation with the

SAN JOAQUIN VALLEY DRAINAGE PROGRAM 
DEPARTMENT OF THE INTERIOR

MANUEL LUJAN, JR., Secretary

U.S. GEOLOGICAL SURVEY

Dallas L. Peck, Director

For additional information write to:

District Chief

U.S. Ceological Survey

Federal Building,

2800 Cottage Way, Room W-2234

Federal Building.

Sacramento, CA 95825
Copies of this report may be purchased from:

U.S. Geological Survey Books and Open-File Reports Section Federal Center, Bldg. 810

Box 25425

Denver, CO 80225 


\section{CONTENTS}

Page

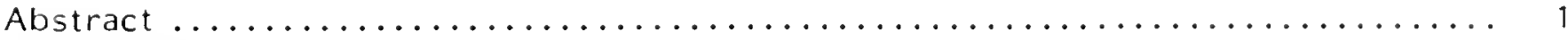

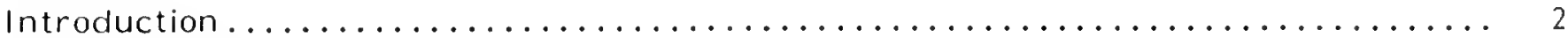

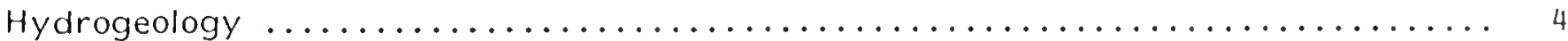

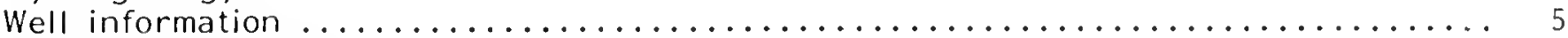

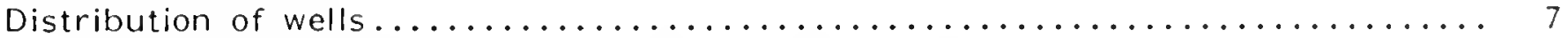

Wells perforated in the semiconfined zone at depths less than or

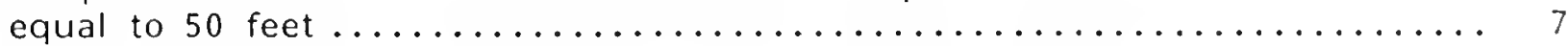

Wells perforated in the semiconfined zone at depths greater

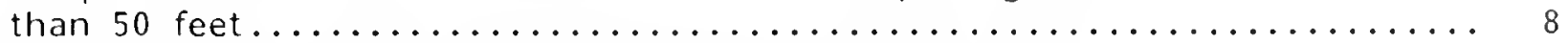

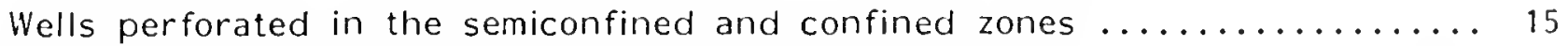

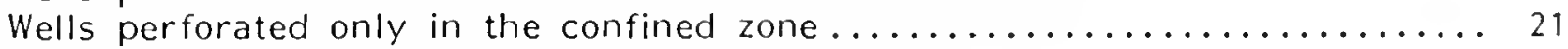

Wells with water levels representative of the semiconfined zone $\ldots \ldots \ldots \ldots \ldots$

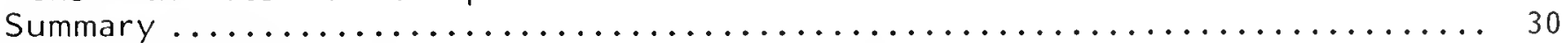

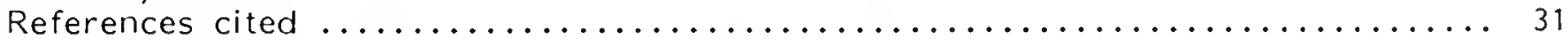

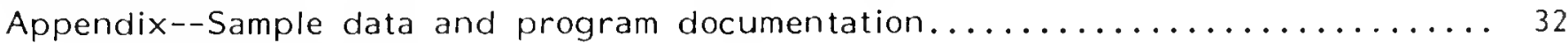

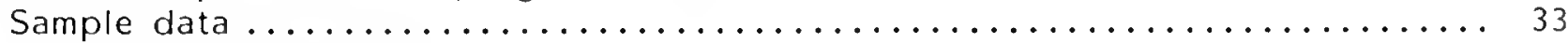

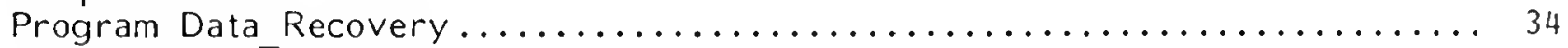

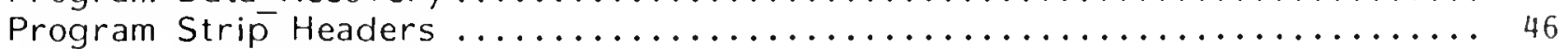

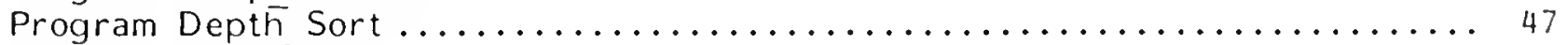

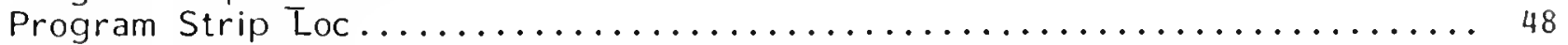

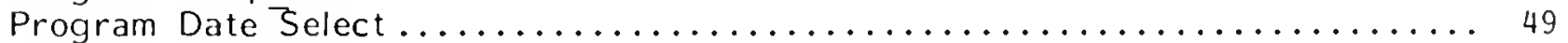

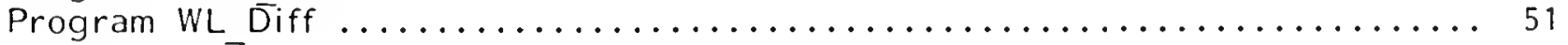

\section{ILLUSTRATIONS}

Figure 1. Map showing location of study area $\ldots \ldots \ldots \ldots \ldots \ldots \ldots \ldots$

2. Generalized hydrogeologic section of the study area perpendicular to the axis of the San Joaquin Valley ............ 4

3. Diagram showing well-numbering system $\ldots \ldots \ldots \ldots \ldots \ldots \ldots \ldots$ 4-7. Maps showing:

4. Areal distribution of wells perforated in the semiconfined zone at depths less than or equal to

5. Areal distribution of wells perforated in the

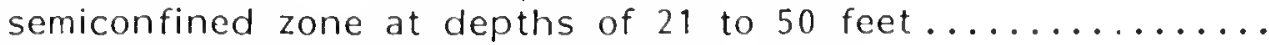

6. Areal distribution of wells perforated in the semiconfined zone at depths greater than 50 feet with perforated lengths in the Coast Range alluvium .......... 10

7. Areal distribution of wells perforated in the semiconfined zone at depths greater than 50 feet with perforated lengths in the Sierran sand 
Figures 8-13. Maps showing:

Page

8. Areal distribution of wells perforated in the semiconfined zone at depths greater than 50 feet with perforated lengths in the Coast Range

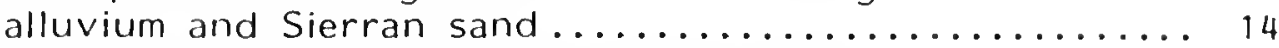

9. Areal distribution of wells perforated in the semiconfined and confined zones with perforated lengths above the Corcoran Clay Member of the Tulare Formation .......................... 17

10. Areal distribution of wells perforated in the semiconfined and confined zones with perforated lengths below the Corcoran Clay Member of the Tulare Formation .......................... 18

11. Areal distribution of wells perforated only in the confined zone with perforated lengths below the Corcoran Clay Member of the Tulare

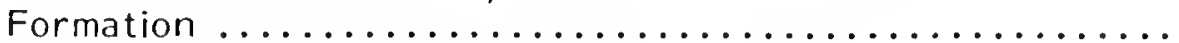

12. Areal distribution of wells with water levels indicative of the water table and dry wells

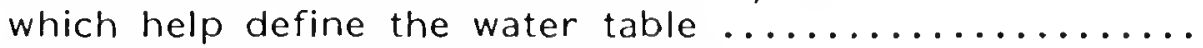

13. Areal distribution of wells perforated below the water table and above the Corcoran Clay Member of the Tulare Formation

\section{TABLES}

Table 1. Description of wells ............................. Page

2. Wells perforated in the semiconfined zone at depths greater than 50 feet with perforated lengths in the Coast Range alluvium ..................................... 11

3. Wells perforated in the semiconfined zone at depths greater than 50 feet with perforated lengths in the Sierran sand ........ 13

4. Wells perforated in the semiconfined zone at depths greater than 50 feet with perforated lengths in the Coast Range

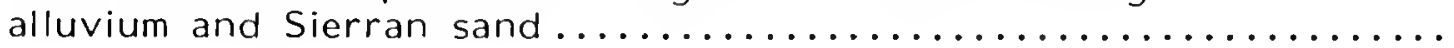


Table 5. Number of wells perforated in the semiconfined zone at depths greater than 50 feet with perforated lengths in the Coast

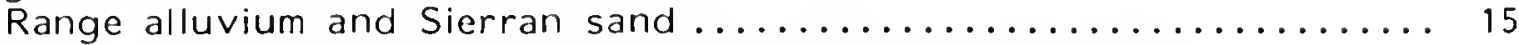

6. Number of wells perforated in the semiconfined and confined zones with perforated lengths above and below the Corcoran Clay Member of the Tulare Formation where Sierran sand

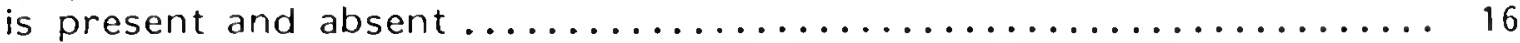

7. Wells perforated in the semiconfined and confined zones with perforated lengths above and below the Corcoran Clay Member of the Tulare Formation ............................. 19

8. Number of wells perforated only in the confined zone with perforated lengths below the Corcoran Clay Member of the Tulare Formation where the Sierran sand is present

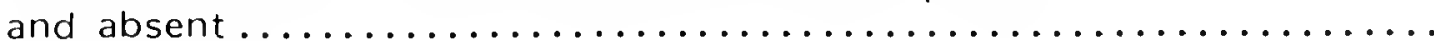

9. Wells perforated only in the confined zone with perforated lengths below the Corcoran Clay Member of the Tulare

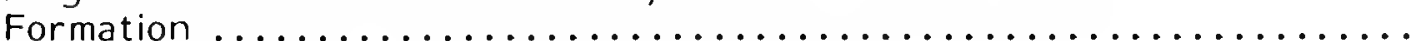

10. Wells with water levels indicative of the water table and dry wells which help define the water table ................... 27

11. Wells perforated below the water table and above the Corcoran Clay Member of the Tulare Formation

\section{CONVERSION FACTORS}

For readers who prefer to use International System (SI) units, rather than the inch-pound terms used in this report, the following conversion factors may be used:

$\begin{array}{lcl}\text { Multiply inch-pound units } & \text { By } & \text { To obtain SI } \\ \text { acre } & 4.047 & \text { square meter } \\ \text { foot }(\mathrm{ft}) & 0.3048 & \text { meter } \\ \text { mile }(\mathrm{mi}) & 1.609 & \text { kilometer }\end{array}$

Sea level: In this report "sea level" refers to the National Geodetic Vertical Datum of 1929 (NGVD of 1929)--a geodetic datum derived from a general adjustment of the first-order level nets of both the United States and Canada, formerly called Sea Level Datum of 1929. 

DISTRIBUTION OF WELLS IN THE CENTRAL PART OF THE

WESTERN SAN JOAQUIN VALLEY, CALIFORNIA

By Jo Ann M. Gronberg, Kenneth Belitz, and Steven P. Phillips

\begin{abstract}
Information from 5,860 wells in the central part of the western San Joaquin Valley, California, was collected from several sources and compiled into a common data base. Only 2,547 wells had sufficient information for classification into four categories based on the hydrogeology: wells perforated in the semiconfined zone at depths less than or equal to 50 feet, wells perforated in the semiconfined zone at depths greater than 50 feet, wells perforated in the semiconfined and confined zones, and wells perforated only in the confined zone. Additionally, wells perforated in the semiconfined zone at depths greater than 50 feet were classified by the type of deposits in which they were perforated (Coast Range alluvium or Sierran sand). A computerized data base system was developed to manage well information and to facilitate characterizing the nature and distribution of the wells.

Wells perforated in the semiconfined zone at depths less than or equal to 50 feet are evenly distributed over part of the study area underlain by shallow ground water. These wells generally are used as observation wells.

Most wells perforated in the semiconfined zone at depths greater than 50 feet are perforated in the Sierran sand. This concentration of wells perforated in the Sierran sand indicates a tendency for using the Sierran sand, where it exists, as a source of water.

There are 533 wells perforated in both the semiconfined and confined zones and 410 wells perforated only in the confined zone. Most of these wells are upslope of the valley trough in areas where the Sierran sand is not present. Wells perforated only in the confined zone are concentrated near the creeks.
\end{abstract}




\section{INTRODUCTION}

The San Joaquin Valley, California, is one of the most productive agricultural areas in the United States. However, agriculture in large parts of the valley may be adversely affected by high levels of selenium and other soluble trace elements that occur in soil, ground water, and agricultural drain water. The occurrence and movement of soluble chemical constituents is related to the movement of ground water in which they are dissolved. Thus, the ground-water flow system must be understood in order to manage these constituents.

Many wells have been drilled in the San Joaquin Valley to supply water for agriculture and to monitor water levels. The large data base available for these wells (for example, water levels, depth, construction information, use) is variable in detail and quality. The large quantity of inconsistent data is difficult to use, especially when specific information is needed. A key component in developing an understanding of the ground-water flow system is the compilation of information from these wells and the synthesis of that information to characterize the system.

This report presents an overview of the compilation and synthesis of information from wells in a part of the San Joaquin Valley, California. The study area is between Los Banos and Kettleman City in the central part of the valley (fig. 1). It is bounded on the west by the Coast Ranges and on the east by the San Joaquin River and the Fresno Slough. Some of the wells included in this report are outside the boundaries of the study area.
Well information collected from several agencies was compiled into a common data base. These wells then were classified into categories based on the hydrogeology of the ground-water flow system. Each category is represented by a location map and a well list. The map illustrates the areal distribution of the wells in a category. It is not meant to provide construction information for a particular well. Instead, the well list can be used in conjunction with the computer programs provided in this report to access and retrieve specific information for a particular well without manually searching through the voluminous data base. The actual data are not presented but are available on request for review at the California district office of the U.S. Geological Survey.

The distribution of the wells is used further to compile information about the ground-water flow system and drilling practices in the western San Joaquin Valley. This information can be used as a starting point for further hydrologic investigations in the San Joaquin Valley.

This study is part of a comprehensive investigation by the U.S. Geological Survey of the hydrology and geochemistry of the San Joaquin Valley. The studies are being done as part of the Regional Aquifer System Analysis Program of the U.S. Ceological Survey in cooperation with the San Joaquin Valley Drainage Program. The authors would like to thank the following agencies for providing data and assistance: U.S. Bureau of Reclamation, California Department of Water Resources, and Westlands Water District. 


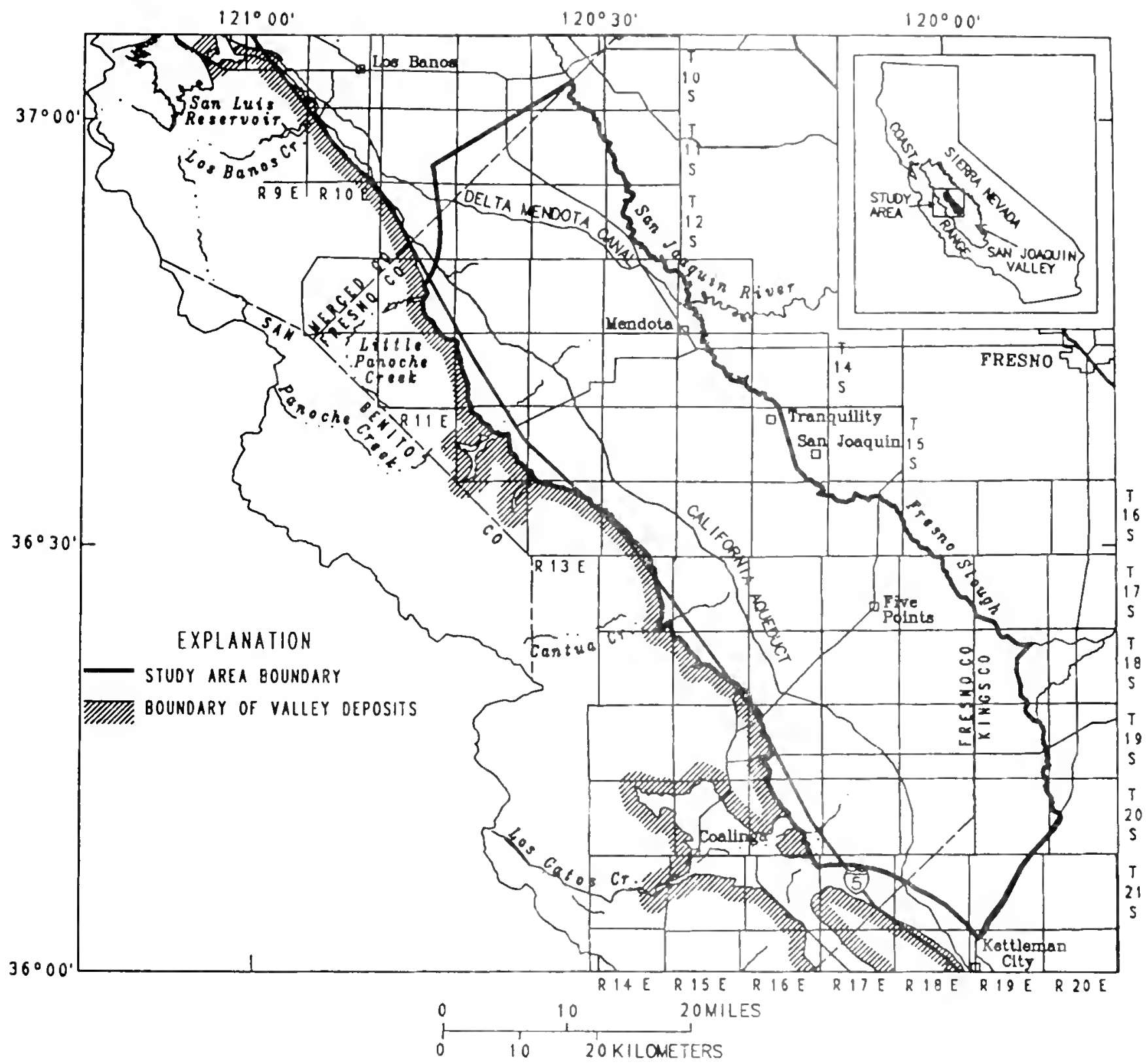

Figure 1. Location of study area. 


\section{HYDROGEOLOGY}

The San Joaquin Valley is underlain by several thousand feet of unconsolidated sediments. The upper several hundred to more than 1,000 feet of these sediments contain primarily fresh ground water (Gilliom and others, 1989, p. 13). The Pleistocene Corcoran Clay Member of the Tulare Formation divides the ground-water flow system into an upper semiconfined zone and a lower confined zone. In the semiconfined zone, three hydrogeologic units can be recognized: Coast Range alluvium, Sierran sand, and flood-basin deposits (fig. 2).

The Coast Range alluvium consists of poorly sorted alluvial-fan deposits derived from the Coast Ranges to the west. The thickness ranges from 850 feet near the Coast Ranges to 0 feet along the valley trough (Page, 1986). Textures range from more than 80 percent sand and gravel in the fanhead regions to more than 80 percent silt and clay in the distal regions (Laudon and Belitz, 1989).

As the Coast Range alluvium thins to the east, it interfingers with sediment derived from the Sierra Nevada. At depth, the Sierran deposits are primarily well-sorted micaceous sand. The Sierran sand is as much as 500 feet thick in the valley trough and thins to the east and west.

Flood-basin deposits form a thin layer (5 to 35 feet thick) over the Sierran sand in the valley trough. They are primarily composed of clays derived from the Coast Ranges and the Sierra Nevada.

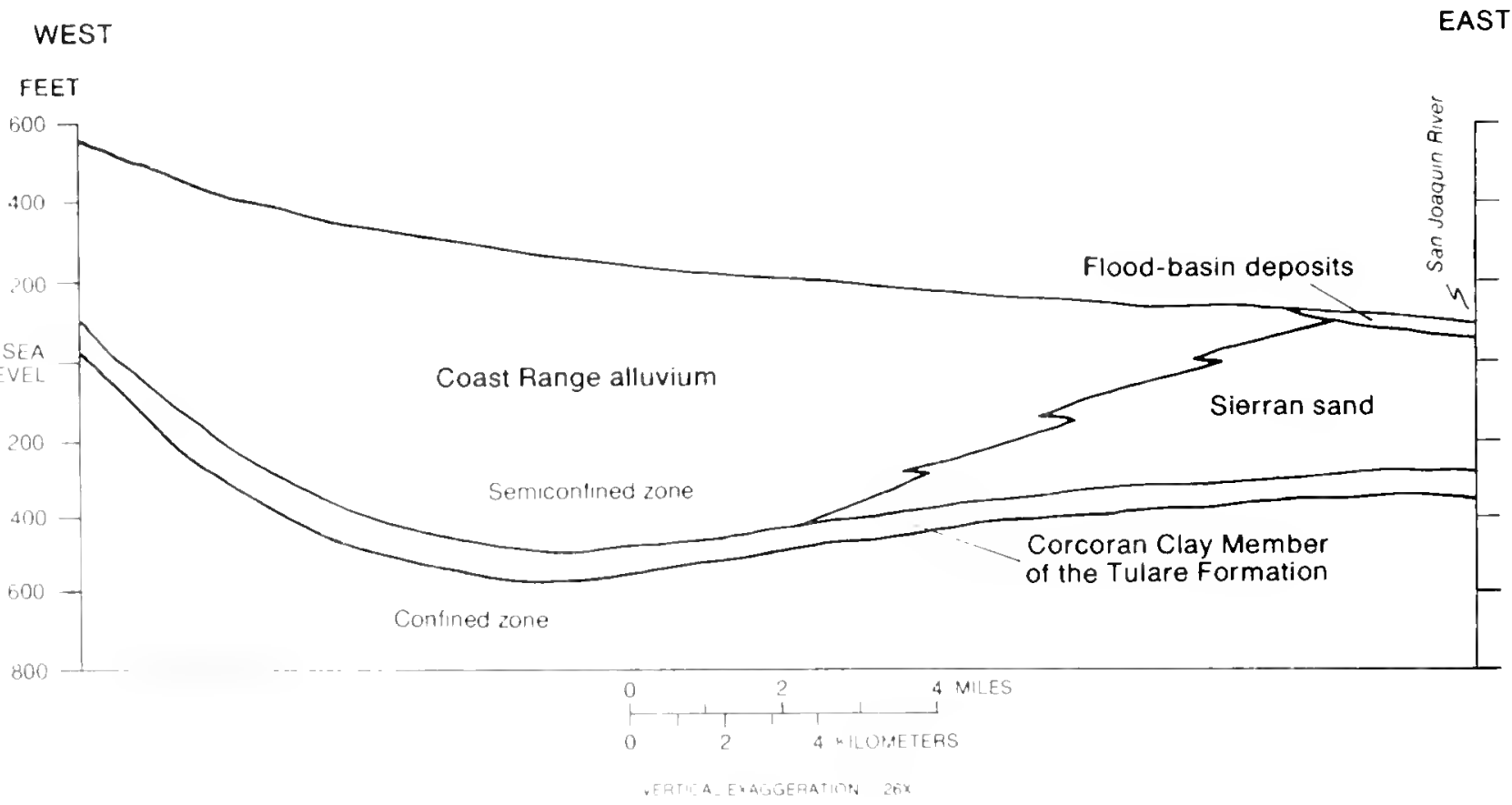

Figure 2. Generalized hydrogeologic section of the study area perpendicular to the axis of the San Joaquin Valley. 
The Corcoran Clay Member of the Tulare Formation, which separates the semiconfined and confined zones, consists of silty clay to clayey silt in the upper two-thirds of the unit, and sandsilt-clay to clayey silt in the lower one-third (Bull, 1975). The base of the unit ranges from more than 850 feet in depth along the Coast Ranges to 400 feet along the valley trough (Bull and Miller, 1975). The Corcoran ranges in thickness from 20 to 120 feet (Miller and others, 1971).

The lower confined zone below the Corcoran consists of poorly consolidated flood-plain, deltaic, alluvial-fan, and lacustrine deposits of the Tulare Formation. The confined zone extends several hundred feet below the Corcoran before saline water is encountered.

For more information on the hydrogeology of the study area see Belitz (1988) and Gilliom and others (1989).

\section{WELL INFORMATION}

Well information was assembled from several sources: the U.S. Bureau of Reclamation, California Department of Water Resources, Westlands Water District, and the U.S. Geological Survey. Additional information on well depths and perforations was obtained from the Survey's Ground Water Site Inventory data base, well logs from the Survey's files, and information found in Ireland (1963) and LeBlanc (1970).

The wells are identified according to their location in the rectangular system for the subdivision of public land, the Public Land Survey System. The identification consists of the township number, north or south: the range number. east or west: and the section number.
The section is further subdivided into 16 tracts of 40 acres each consecutively lettered (excepting 1 and $O$ ), beginning with $A$ in the northeast corner of the section and progressing in a sinusoidal manner to $R$ in the southeast corner. Wells within the 40-acre tract are numbered sequentially. An example of a well identification is 13S13E13D01. All wells are reinforced to the Mount Diablo base line and meridian. This wellnumbering system is shown in figure 3 .

For consistency, all data were converted to a format used by the Bureau of Reclamation. The format consists of a well header, followed by water-level measurements. Each well header has a location by township, range, section, and quarter-quarter section, and when available, well depth and period of record for the water-level measurements. Waterlevel entries include measuring-point altitude, land-surface altitude, depth to water from measuring point, depth to water from land surface, and watersurface altitude. Water levels are recorded on several different schedules: monthly. quarterly, biannually, or miscellaneous measurements. The entire data base can be examined at the California District office of the U.S. Geological Survey.

Because of the large quantity of data, several Fortran programs were written to systematically search the data base for wells by location, depth, and period of record. These programs (Data Recovery, Strip Headers, Depth Sort, Strip Loc, Date Select, WL Diff) are described and presented in the Appendix. Sample Data and Program Documentation, at the back of this report.

A geographic information system data base also was created ?) store wellconstruction information and to provide a spatial reference for the data. 


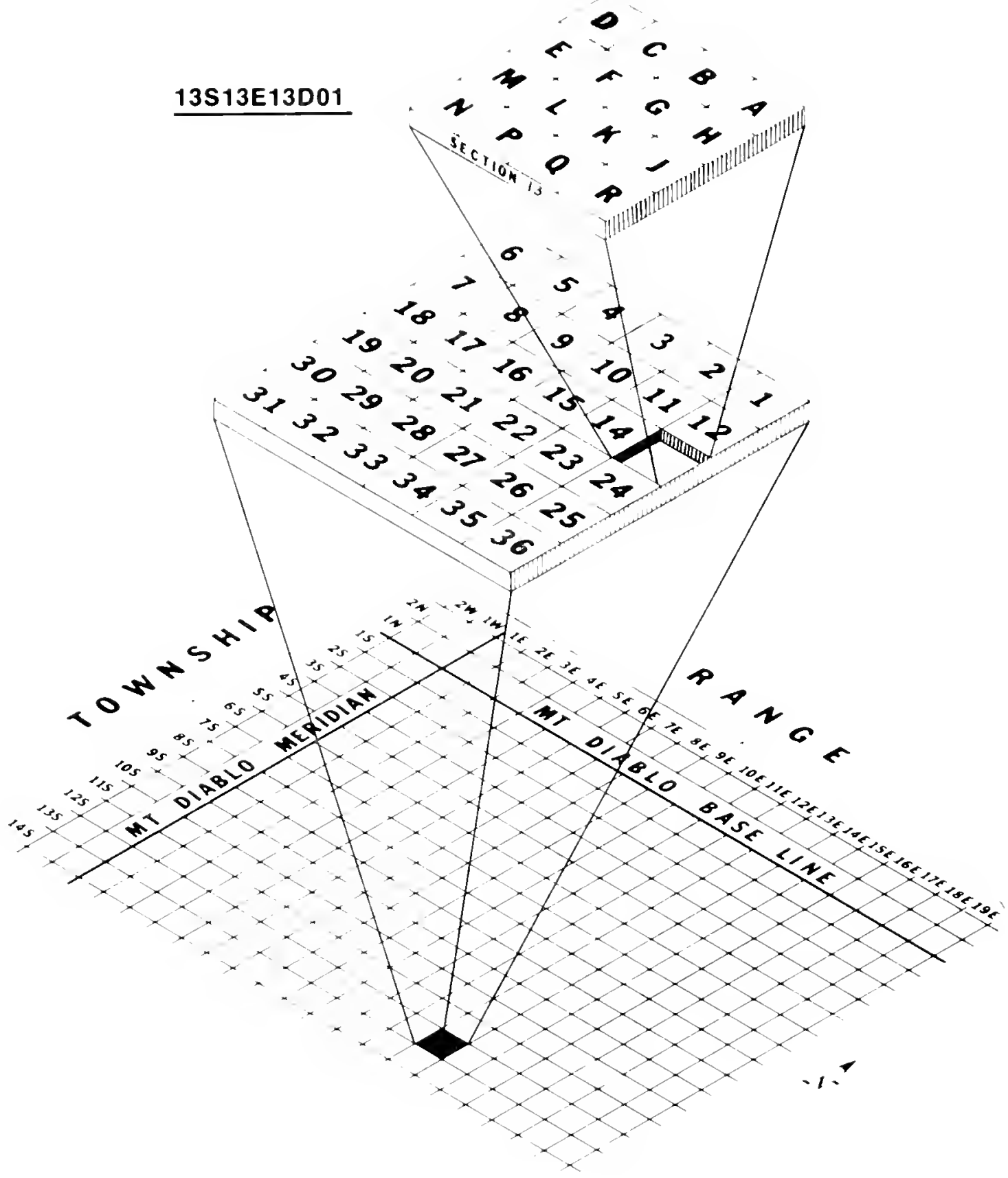

Figure 3. Wel-numbering system. 


\section{DISTRIBUTION OF WELLS}

The data base contains information from 5,860 wells. This report focuses on wells within the previously described study area, which limits the data set to 4,715 wells. Of these 4,715 wells, 3,050 wells have depth information and 1,407 have perforated length information. However, only 2,547 wells have sufficient information on depth and perforated length for classification into hydrologically useful categories. Four such categories were identified: (1) wells perforated in the semiconfined zone at depths less than or equal to 50 feet, (2) wells perforated in the semiconfined zone at depths greater than 50 feet, (3) wells perforated in the semiconfined and confined zones, and (4) wells perforated only in the confined zone. (See table 1 for additional detail). An additional 253 wells were drilled below the Corcoran Clay Member of the Tulare Formation, but perforation information was not available to help determine which zones they were perforated in. Also, 267 wells are in areas where the Corcoran is not present.

A discussion of these categories is presented in the following sections. Each section describes the wells and their location in the semiconfined and confined zones. Maps of well locations are included to illustrate the areal distribution of the wells. Complete listings of wells in each category can be used in conjunction with the programs provided in the Appendix to retrieve information on the specific wells.

\section{Wells Perforated in the Semiconfined}

Zone at Depths Less Than or

Equal to 50 Feet

There are 1,114 wells perforated in the semiconfined zone at depths less than or equal to 50 feet. Most of these
TABLE 1.--Description of wells

Description of wells

Number

of wells

Wells perforated in the semi-

confined zone at depths less

than or equal to 50 feet

generally used as observation

wells.

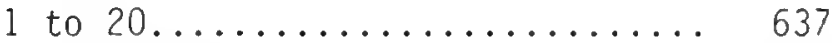

21 to $50 \ldots \ldots \ldots \ldots \ldots \ldots \ldots . \ldots . \ldots . \ldots . \ldots 17$

Subtotal............. $\overline{1,114}$

Wells perforated in the semi-

confined zone at depths greater

than 50 feet.

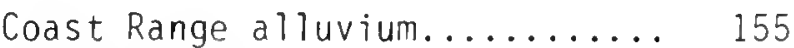

Sierran sand............... 275

Coast Range alluvium and

Sierran sand.............. 21

No perforation information..... 39

Subtotal............... $\overline{490}$

Wells perforated in the semi-

confined and confined zones..... 533

Wells perforated only in the

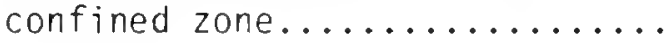

410

Total .............. 2,547

shallow wells are observation wells. Numerous wells at depths less than or equal to 20 feet were installed by the U.S. Bureau of Reclamation and are maintained by the Westlands Water District to monitor the water table. These wells are perforated over the entire length of the well (Westlands Water District, oral commun., 1987). Many wells at depths of about 50 feet are along the California Aqueduct. The western limit of the 20-foot wells (fig. 4) reflects the upslope limit of the area underlain by a water table within 20 feet of the land surface. 


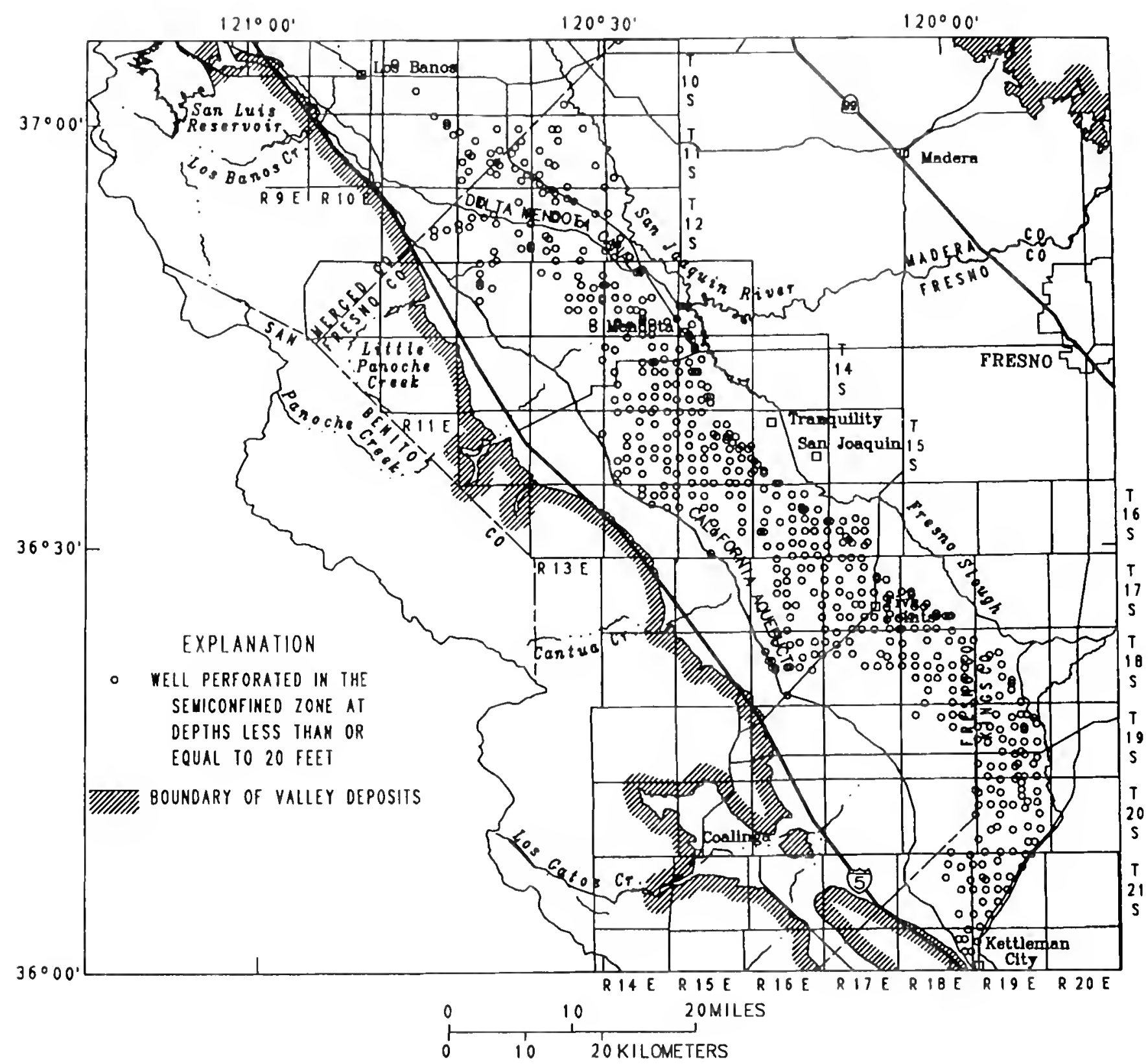

Figure 4. Areal distribution of wells perforated in the semiconfined zone at depths less than or equal to 20 feet.

The 637 wells perforated within 20 feet of the land surface are uniformly distributed over the area where the depth to water is less than 20 feet. Figure 5 indicates a sparser coverage of wells at depths of 21 to 50 feet over the study area, with a concentration of wells along the California Aqueduct.
Wells Perforated in the Semiconfined Zone at Depths Greater Than 50 Feet

There are 490 wells perforated in the semiconfined zone at depths greater than 50 feet. These wells are classified by the type of deposits they are perforated in. 


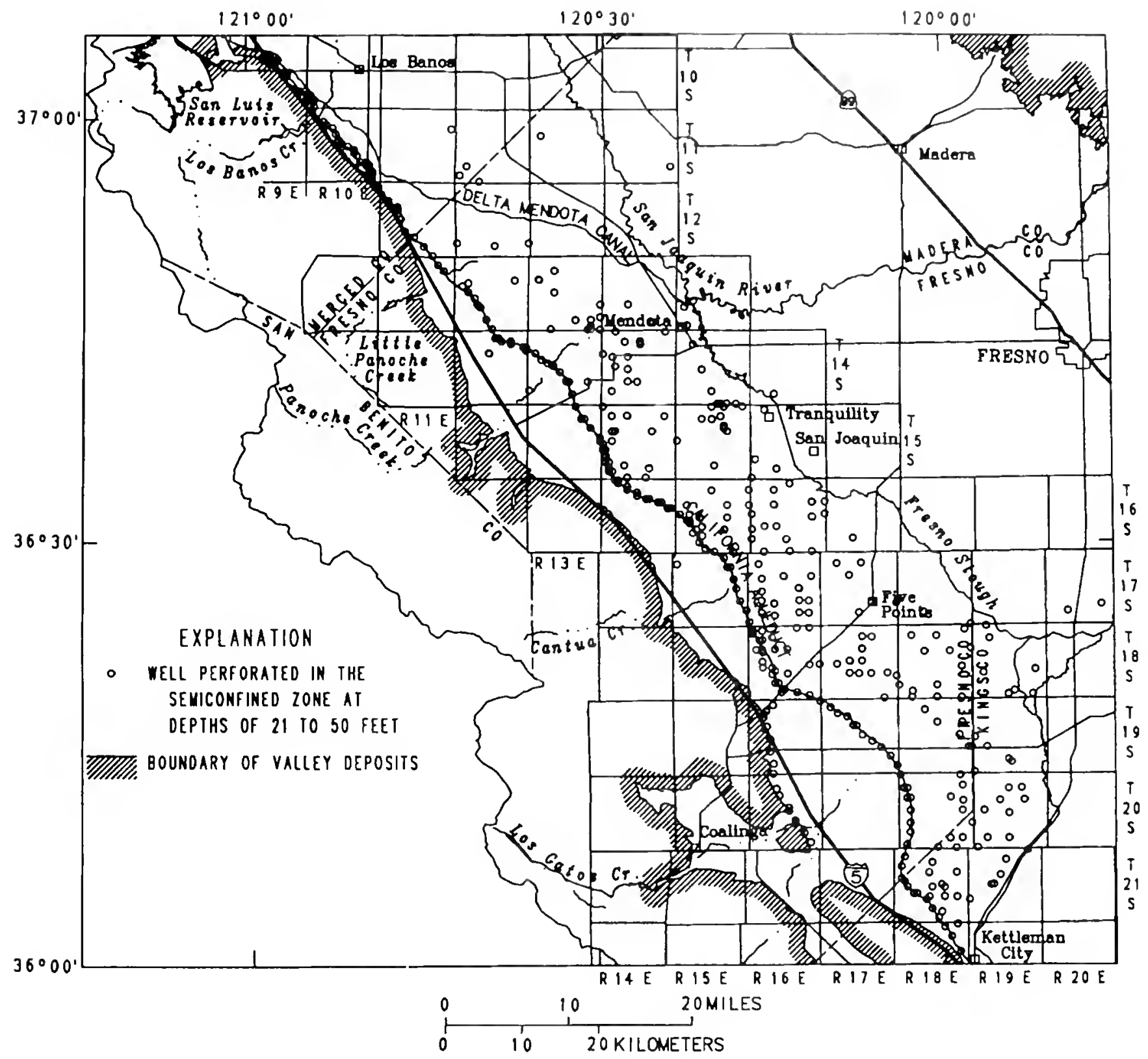

Figure 5. Areal distribution of wells perforated in the semiconfined zone at depths of 21 to 50 feet.

Perforated lengths of the wells were compared to thickness maps of the Coast Range alluvium and Sierran sand (Miller and others, 1971). Because the floodplain deposits generally are less than 50 feet thick, an attempt was not made to separate wells perforated in the flood-plain deposits from those perforated in the Sierran sand. There are 155 wells perforated in Coast Range alluvium (fig. 6: table 2), 275 in Sierran sand (fig. 7; table 3), 21 in both (fig. 8; table 4), and 39 wells without sufficient information to determine which deposits they perforated. 


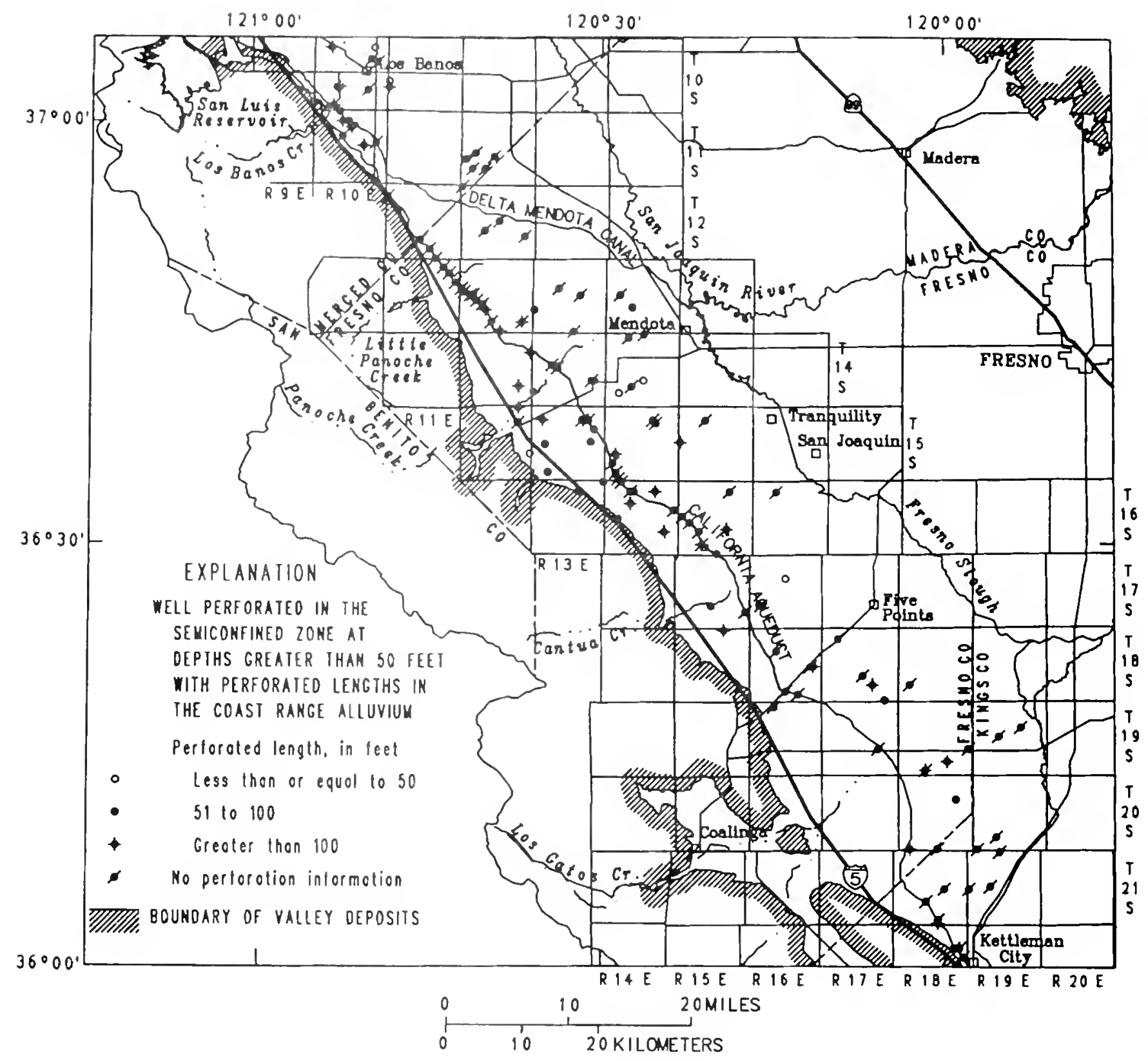

Figure 6. Areal distribution of wells perforated in the semiconfined zone at depths greater than 50 feet with perforated lengths in the Coast Range alluvium. 
TABLE 2.--Wells perforated in the semiconfined cone at depths greater than 50 feet with perforated lengths in the Coast Range alluvium

\begin{tabular}{|c|c|c|c|c|c|}
\hline $\begin{array}{l}\text { 10S1OEO2R01 } \\
1 \text { OS1OEOSP01 } \\
\text { 10S1OE11001 } \\
\text { 10S1OE13D01 }\end{array}$ & $\begin{array}{l}\text { 12S11E34R02 } \\
12 \text { S12E06001 } \\
12 \text { S12E1SN01 } \\
12 S 12 E 20 J 01\end{array}$ & $\begin{array}{l}13 S 13 E 34 P 01 \\
13 S 14 E 17 N 02 \\
13 S 14 E 21 N 04 \\
14 S 12 E 12 J 01\end{array}$ & $\begin{array}{l}\text { 1SS13E14R01 } \\
15513 E 20001 \\
15513 E 32 F 01 \\
15514 E 1 O A 01\end{array}$ & $\begin{array}{l}\text { 15S1SE18P02 } \\
16 S 1 S E 19401 \\
16 S 15 E 22 R 01 \\
15 S 1 S E 29 B 02\end{array}$ & $\begin{array}{l}19 S 16 \mathrm{EOSHO4} \\
19 \mathrm{S17E23PO1} \\
19 S 18 \mathrm{E} 24001 \\
19518 \mathrm{E} 27 \mathrm{RO} 1\end{array}$ \\
\hline $\begin{array}{l}\text { 10S1OE26F01 } \\
10 S 10 E 28 D 01 \\
10 S 10 E 29 A 01 \\
10 S 10 E 32 \mathrm{KO} 2\end{array}$ & $\begin{array}{l}12 S 12 E 25002 \\
13 S 11 E 02 K 01 \\
13 S 11 E 12001 \\
13 S 11 E 12001\end{array}$ & $\begin{array}{l}14 \mathrm{~S} 12 \mathrm{E} 26 \mathrm{HO} 1 \\
14513 \mathrm{E} 16 \mathrm{NO} 4 \\
14 \mathrm{S13ET} 7 \mathrm{KO} 3 \\
14 \mathrm{~S} 13 \mathrm{E} 23 \mathrm{RO}\end{array}$ & $\begin{array}{l}\text { 15S14E1OA02 } \\
15 S 14 E 10 A 03 \\
15 S 14 E 11 E 01 \\
15 S 14 E 19 R 01\end{array}$ & $\begin{array}{l}16 S 15 E 32 \mathrm{AOO} \\
16 S 15 E 32 \mathrm{A05} \\
16 S 15 E 33 \mathrm{EO} 1 \\
16 S 15 E 34 \mathrm{NOS}\end{array}$ & $\begin{array}{l}19 S 18 E 33 M 01 \\
19 S 18 E 33 M 02 \\
19 S 19 E 1 S A 02 \\
19 S 19 E 16 N 02\end{array}$ \\
\hline $\begin{array}{l}\text { 1OS1OE32N01 } \\
10 S 11 E 19 M 01 \\
10 S 11 E 30001 \\
11 S 10 E 04001\end{array}$ & $\begin{array}{l}13 S 11 E 12002 \\
13 S 12 E 17 N O 1 \\
13 S 12 E 18 E 01 \\
13 S 12 E 18 E 02\end{array}$ & $\begin{array}{l}14 \mathrm{~S} 13 \mathrm{E} 24 \mathrm{NO} 2 \\
14 \mathrm{~S} 13 \mathrm{E} 24 \mathrm{NOS} \\
14 \mathrm{~S} 13 \mathrm{E} 24 \mathrm{NO6} \\
14 \mathrm{~S} 13 \mathrm{E} 24 \mathrm{NO}\end{array}$ & $\begin{array}{l}\text { 1SS14E30KO1 } \\
15 S 14 E 31 \mathrm{HO2} \\
15 S 14 E 31 \mathrm{HO} \\
15 S 14 \mathrm{E} 32 \mathrm{NO} 3\end{array}$ & $\begin{array}{l}\text { 16S16E05RO2 } \\
17 S 1 S E 25 K 01 \\
17 S 1 S E 28 A 01 \\
17 S 16 E 09 R 02\end{array}$ & $\begin{array}{l}\text { 20S18E:1001 } \\
20 S 18 E 31 R 01 \\
20 S 18 E 34 N 01 \\
20 S 18 E 34 N 02\end{array}$ \\
\hline $\begin{array}{l}11 \text { S1OEO4P01 } \\
11 \text { S1OEO4RO1 } \\
11 \text { S1OE1OEO1 } \\
11 \text { S1OE } 3 M 01\end{array}$ & $\begin{array}{l}13 S 12 E 18 L 01 \\
13 S 12 E 18 R 01 \\
13512 E 20002 \\
13512 E 20 F 02\end{array}$ & $\begin{array}{l}14 S 13 \text { E3ONO4 } \\
14 \mathrm{~S} 14 \mathrm{EO} 3 \mathrm{DO} 1 \\
14 \mathrm{~S} 14 \mathrm{EOSHO1} \\
14 \mathrm{~S} 14 \mathrm{E} 22 \mathrm{NO} 4\end{array}$ & $\begin{array}{l}\text { 1SSISE09D0S } \\
15 S 15 E 18 N O 1 \\
16 S 13 E 01 A 01 \\
16 S 13 E 03 R 01\end{array}$ & $\begin{array}{l}\text { 17S16E30A03 } \\
18 S 15 E 03 \mathrm{AO} 2 \\
18516 E 09 N 01 \\
18 S 16 E 23 \mathrm{AO} 2\end{array}$ & $\begin{array}{l}\text { 20S19E29R01 } \\
\text { 20S19E31P02 } \\
21 S 18 E 23005 \\
21 S 18 E 28 B 01\end{array}$ \\
\hline $\begin{array}{l}11 \mathrm{~S} 10 \mathrm{E} 14 \mathrm{NO} 1 \\
11 \mathrm{~S} 10 \mathrm{E} 16 \mathrm{CO} 1 \\
11 \mathrm{~S} 12 \mathrm{E} 19 \mathrm{PO} 1 \\
11 \mathrm{~S} 12 \mathrm{E} 20 \mathrm{E} 01\end{array}$ & $\begin{array}{l}\text { 13S12E20J01 } \\
13512 \text { E20R02 } \\
13512 E 28003 \\
13512 E 33 B 04\end{array}$ & $\begin{array}{l}14 \mathrm{~S} 14 \mathrm{E} 28 \mathrm{EO} 2 \\
14 \mathrm{~S} 14 \mathrm{E} 29 \mathrm{NO} 2 \\
15 \mathrm{~S} 12 \mathrm{EO} 2 \mathrm{AO} 1 \\
15 \mathrm{~S} 12 \mathrm{E} 1 \mathrm{HO} 01\end{array}$ & $\begin{array}{l}16514 \mathrm{E} 02 \mathrm{NO} 1 \\
16 \mathrm{~S} 14 \mathrm{E} 04 \mathrm{NO} 4 \\
16514 \mathrm{E} 04 \mathrm{PO} 1 \\
16 \mathrm{~S} 14 \mathrm{E} 05 \mathrm{CO} 1\end{array}$ & $\begin{array}{l}18516 E 24002 \\
18516 E 33 \mathrm{AO} 4 \\
18516 E 34 \mathrm{HO2} \\
18517 \mathrm{E} 05 \mathrm{NO} 4\end{array}$ & $\begin{array}{l}21 \mathrm{~S} 18 \mathrm{E} 34 \mathrm{KO} 2 \\
21 \mathrm{~S} 18 \mathrm{E} 34001 \\
21 \mathrm{~S} 19 \mathrm{E} 04 \mathrm{BO} 1 \\
21 \mathrm{~S} 19 \mathrm{E} 17 \mathrm{R} 02\end{array}$ \\
\hline $\begin{array}{l}11 \mathrm{S12E21KO1} \\
11 \mathrm{~S} 12 \mathrm{E} 29 \mathrm{~J} 01 \\
11 \mathrm{~S} 12 \mathrm{E} 30 \mathrm{O} 03 \\
12 \mathrm{~S} 11 \mathrm{E} 06 \mathrm{NO} 1\end{array}$ & $\begin{array}{l}13 S 12 E 34 P 02 \\
13 S 12 E 36003 \\
13 S 12 E 36004 \\
13513 E 15 R 01\end{array}$ & $\begin{array}{l}15 S 12 E 11 \mathrm{HO} 2 \\
15 S 12 E 24 R O 1 \\
15 S 13 E 07 A O 1 \\
15 S 13 E 11 B 02\end{array}$ & $\begin{array}{l}16 S 14 \mathrm{EO9NO} 1 \\
16 \mathrm{~S} 14 \mathrm{E} 13 \mathrm{CO} 01 \\
16514 \mathrm{E} 13 \mathrm{CO} 04 \\
16514 \mathrm{E} 20001\end{array}$ & $\begin{array}{l}\text { 18S17E22NO1 } \\
18517 E 27 J 01 \\
18 S 17 E 35 R 02 \\
18518 E 30 J 02\end{array}$ & $\begin{array}{l}\text { 21S19E19001 } \\
22 S 18 E 11 R 01 \\
22 S 18 E 12 P 01 \\
22 S 18 E 12 P 02\end{array}$ \\
\hline $\begin{array}{l}12 S 11 E 28 \mathrm{CO} 1 \\
12 S 11 E 34 \mathrm{CO} 1\end{array}$ & $\begin{array}{l}13513 E 16 E 01 \\
13513 E 30001\end{array}$ & $\begin{array}{l}15 S 13 E 11003 \\
15513 E 12 N 04\end{array}$ & $\begin{array}{l}16514 E 26 A 01 \\
16515 E 02 N 02\end{array}$ & $\begin{array}{l}19516 \text { EOSHO2 } \\
19 S 16 \text { EOSHO3 }\end{array}$ & $22 S 18 E 13 K 01$ \\
\hline
\end{tabular}

Many wells perforated in the Coast Range alluvium are along the California Aqueduct. A large number (41) of these are observation wells monitoring the aqueduct. These wells range in depth from 50 to 60 feet and 190 to 210 feet. The remaining 114 wells tap the Coast Range alluvium as a source of water.
The average perforation length for these wells is 114 feet. Most of the wells have perforated lengths less than 100 feet (table 5). The small number and relatively short perforated lengths of these wells reflects the relatively low transmissivity of the Coast Range alluvium. 


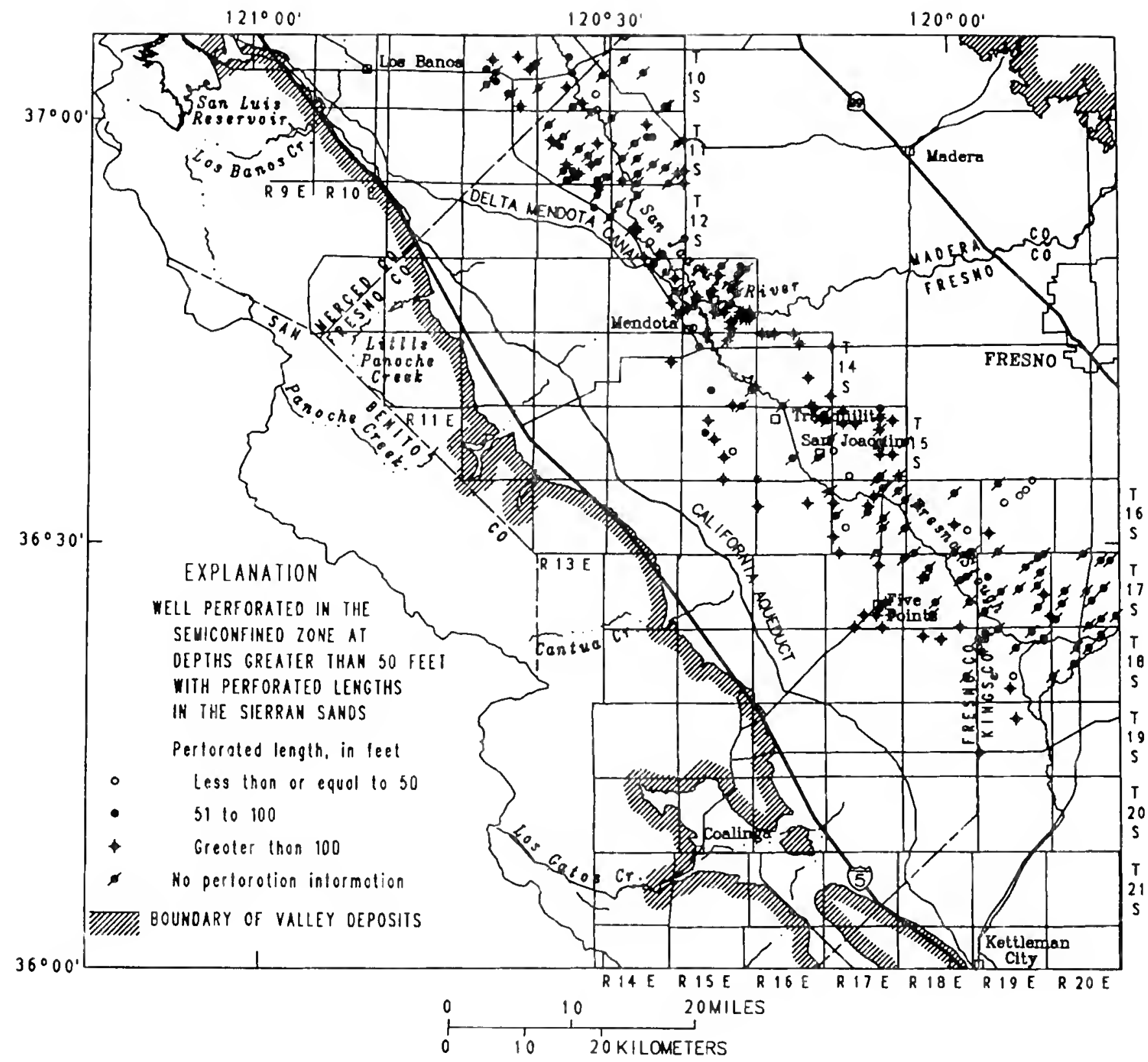

Figure 7. Areal distribution of wells perforated in the semiconfined zone at depths greater than 50 feet with perforated lengths in the Sierran sand. 
TABLE 3.--Wells perforated in the semiconfined zone at depths greater than 50 feet with perforated lengths in the Sierran sand

\begin{tabular}{|c|c|c|c|c|c|}
\hline 10S12E09P01 & 11S14E13L01 & 13S15E14MO1 & 14S16E33NO1 & 16S17E $16 A 01$ & 17S19E07R01 \\
\hline 10S12E11L01 & 11S14E15C01 & 13S15E14P02 & 14S16E3SP01 & 16S17E16J01 & 17S19E10A01 \\
\hline $10 S 12 E 13 A 01$ & $11514 E 16 A 01$ & 13S1SE16L01 & 14S16E36A01 & 16S17E 18P01 & 17S19E 12:401 \\
\hline 10S12E13L01 & 11S14E17J01 & 13S1SE18M01 & 15S1SE09002 & 16S17E2ONO2 & 17S19E14J01 \\
\hline $10 S 12 \mathrm{E} 16001$ & 11S14E19R01 & 13S1SE19R01 & 15S15E09003 & 16S17E23002 & 17S19E $15 P 01$ \\
\hline 10S12E17J01 & 11S14E21N01 & 13S1SE20001 & 15S15E16K01 & 16S17E23NO1 & 17S19E15P02 \\
\hline 1OS12E20R01 & 11S14E2SR01 & 13S1SE21001 & 15S15E $16 \mathrm{~K} 02$ & 16S17E24R01 & $17 S 19 E 20 A 01$ \\
\hline 10S12E21K01 & 11S14E26L01 & 13S1SE22J01 & 1SS1SE17A01 & 16S17E30M01 & 17S19E24F01 \\
\hline 10S12E27J01 & $11514 E 27 F 01$ & 13S1SE2SF 01 & 1SS1SE34P01 & 16S17E31001 & 17S19E26HO1 \\
\hline 10S12E27K01 & 11S14E31 J01 & 13S1SE25L01 & 15515E23M01 & 16S17E34001 & 17S19E30K01 \\
\hline 10S12E3SKO 1 & 11S14E33D01 & 13S1SE25NO1 & 1SS1SE27C01 & 16S18E10A01 & $17 S 19 E 36 A 01$ \\
\hline 10S13E09K01 & 11S14E33NO1 & 13S1SE2SNO2 & 1SS16E01L01 & $16 \mathrm{~S} 18 \mathrm{E} 18 \mathrm{~A} 01$ & $17520 E 02 M O 1$ \\
\hline 10S13E16R01 & $11514 E 35001$ & 13S1SE.25P01 & 1SS16E01N01 & 16S18E22J01 & $17520 E 02 J 01$ \\
\hline 10S13E24CO1 & 11S14E36C01 & 13S15E26C01 & 1SS16E02B01 & 16S18E26A02 & $17520 E 04 B 01$ \\
\hline 10S13E26J01 & 11S14E36R01 & 13S1SE26H01 & 1SS16E12B01 & $16 S 18 E 31001$ & 17S20E14P01 \\
\hline $10513 E 28 \mathrm{CO} 1$ & 12S13E02A01 & 13S1SE26K01 & 15S16E $12 \mathrm{CO} 2$ & 16S18E34R01 & 17520 E20001 \\
\hline $10 S 13 E 30001$ & 12S13E02R01 & 13S1SE27F01 & 15S16E13J01 & 16S18E36P01 & 17S20E22P01 \\
\hline 10S13E33J01 & 12S13E 11001 & 13S15E28C01 & $15 S 16 E 26 A 01$ & 16S19E02001 & 17S20E21A01 \\
\hline $10 S 13 E 34 \mathrm{CO} 1$ & 12S13E13J01 & 13S1SE28HO 1 & $15 S 16 E 28 A 02$ & $16 S 19 E 03001$ & 17S20E30A01 \\
\hline 10S13E3SR01 & 12S14E04N01 & $13515 E 30 \mathrm{C} 01$ & 15S16E31N03 & 16S19EOSF01 & 17S20E31F01 \\
\hline 10514E08N01 & 12S14E07K01 & 13S1SE31 J04 & 15S17E03A01 & 16S19E08R01 & 17S20E34NO 1 \\
\hline 10S14E 16Q01 & 12S14E20K01 & 13S1SE34A01 & 1SS17E03R01 & 16S19E10E01 & $17520 E 36 \mathrm{CO} 1$ \\
\hline 10S14E35K01 & 12S14E 20R01 & 13S15E34J01 & 15S17E06H01 & $16 S 19 E 30 \mathrm{CO} 1$ & 17520E3SE01 \\
\hline TOS14E35L01 & 12S14E21M01 & 13S15E34J02 & 15S17E07H01 & 16S19E32P01 & 18S18E03NO 1 \\
\hline $11 \mathrm{~S} 13 \mathrm{E} 02 \mathrm{CO} 1$ & 12S14E2SHO1 & 13515E3500S & 15S17E08H01 & 17S17EO2NO1 & 18518 EOSKO 1 \\
\hline $11 S 13 E 07 A 01$ & 12514E27M01 & $14514 E 13 E 03$ & 15S17E1OR01 & $17517 E 23001$ & 18518E241101 \\
\hline 11S13E14J01 & $12 S 14 E 33001$ & 14S15E03A01 & 15S17E1OR02 & 17S17E25N01 & 18S19E02F02 \\
\hline 11S13E16002 & $12 S 14 E 34 \mathrm{JO} 3$ & 14515E03K02 & 1SS17E11A01 & 17S17E26EO4 & 18S19E01P01 \\
\hline 11S13E17E01 & 12S14E35MO1 & 1451 SE03KO4 & 1SS17E13R01 & 17S17E27R02 & 18S19EOSBO 1 \\
\hline 11S13E17L01 & 13S14E03C01 & 14S15EOSA02 & 15S17E1SR01 & 17517E28R04 & 18S19E06C01 \\
\hline 11S13E17R01 & 13S14E03LO1 & $14 S 15 E 0 S A 03$ & 15S17E19M01 & 17S17E33N01 & 18S19E06NO2 \\
\hline 11S13E22C01 & 13S14E11B01 & 14S1SECSA04 & 15S17E22H01 & 17S17E3SP01 & 18S19E07:02 \\
\hline 11S13E23M01 & 13S14E12L01 & 14S1SEOSAOS & 15S17E22R01 & $17 S 18 E 01 B 01$ & 18S19E06002 \\
\hline 11 S13E26A01 & 13S14E13L01 & $14515 E 08 \mathrm{CO} 4$ & 1SS17E23R01 & 17S18E02A01 & 18S19E $10 M 01$ \\
\hline 11S13E26F01 & 13S14E24M01 & 14S1SE2SHO2 & 15S17E32L03 & $17518 E 06 E 01$ & 18S19E20P02 \\
\hline $11 \mathrm{~S} 13 \mathrm{E} 28 \mathrm{~F} 01$ & 13S14E25HO1 & 14S15E25K01 & 15S17E34J01 & $17 S 18 E 08001$ & 18S19E20P03 \\
\hline 11S13E28N01 & 13S14E2SK01 & 14515E 28L04 & $15 S 17 E 34003$ & 17S18E08R01 & 18S19E21R03 \\
\hline 11S13E28R01 & 13S15E01 N01 & 14S15E35N01 & 15S17E36L01 & $17 \mathrm{~S} 18 \mathrm{E} 09001$ & 18S19E28001 \\
\hline 11S13E33L01 & 13S15E02K01 & 14S15E35R01 & 16S16E01R01 & $17518 \mathrm{E} 12 \mathrm{NO} 1$ & 18S20E02M01 \\
\hline 11S13E34C01 & 13S1SE03M01 & 14S16E03P01 & 16S16E11N02 & $17 S 18 E 14 B 01$ & 18S20E09:101 \\
\hline $11513 E 34$ E01 & $13 S 1$ SEOSO0 1 & 14S16E04A01 & $16516 E 18001$ & $17 S 18 E 21002$ & 18S20E10H01 \\
\hline 11S13E35J01 & 13S1SE09HO1 & 14S16E05C01 & 16S17E01P01 & 17S18E24P01 & 18S20E 17R01 \\
\hline 11S13E36C01 & 13S1SE11B01 & 14S 16E06A01 & 16S17E02MO1 & 17S18E28P01 & 18S20E19NO 1 \\
\hline 11S14E06M1.101 & 13S1SE11F01 & $14 S 16 E 06 \mathrm{CO} 1$ & 16S17E07N01 & $17518 E 35001$ & 19S19E $10 \mathrm{E} 02$ \\
\hline 11S14E09A03 & 13S15E11P01 & 14S16E12A01 & 16S17E1OFO 1 & 17S19E01C01 & 19519E30D01 \\
\hline 11S14E13H01 & 13S1SE14E01 & 14S16E23M0! & 16S17E12J01 & 17S19E02+101 & \\
\hline
\end{tabular}




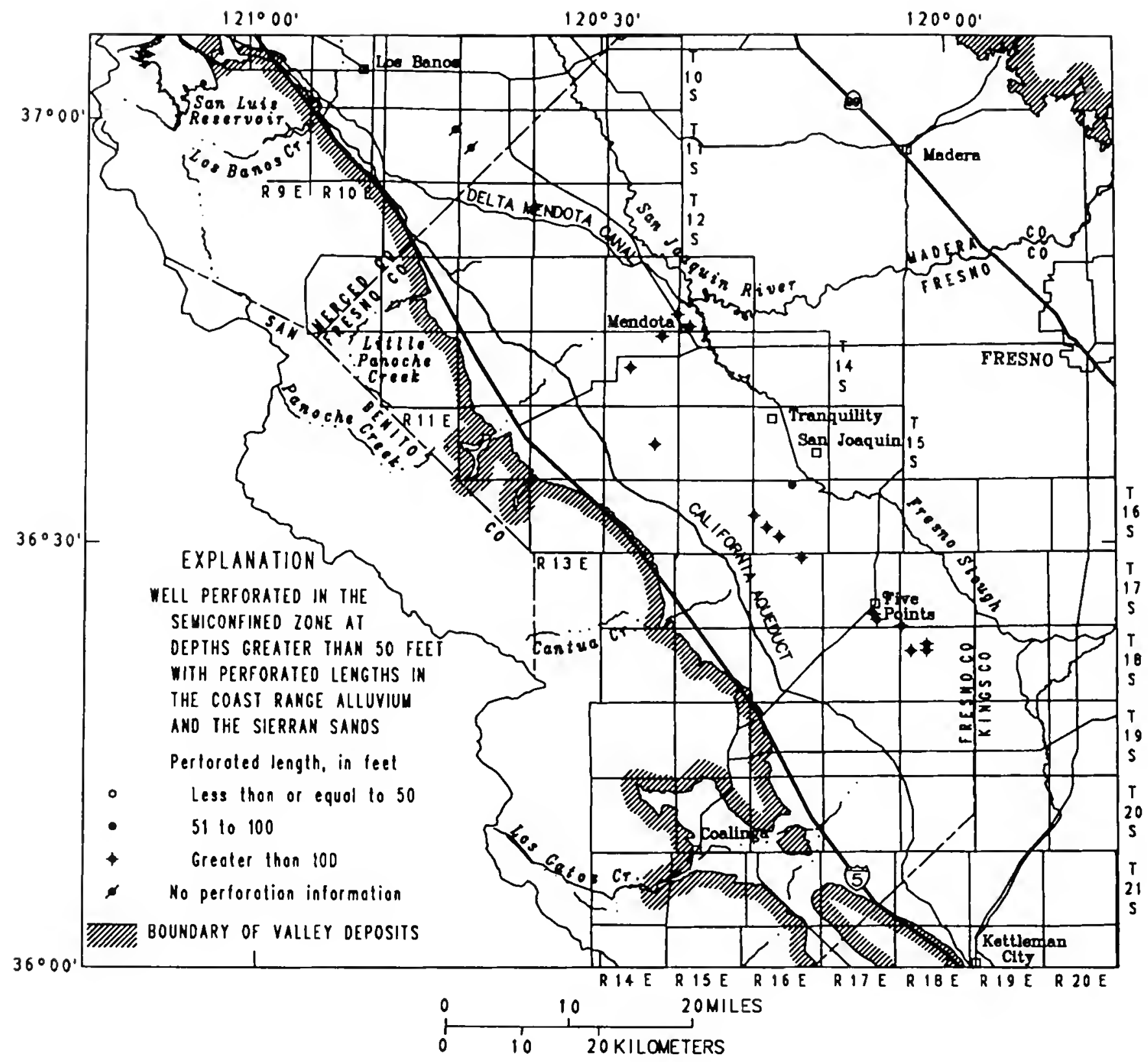

Figure 8. Areal distribution of wells perforated in the semiconfined zone at depths greater than 50 feet with perforated lengths in the Coast Range alluvium and Sierran sand. 
TABLE 4.--Wells perforated in the semiconfined zone at depths greater than 50 feet with perforated lengths in the Coast Range alluvium and Sierran sand

\begin{tabular}{|c|c|c|}
\hline $\begin{array}{l}11 \mathrm{~S} 11 \mathrm{E} 12 \mathrm{KO} 3 \\
11 \mathrm{~S} 12 \mathrm{E} 19 \mathrm{A01} \\
12 \mathrm{~S} 13 \mathrm{E} 07 \mathrm{NO} \\
13 \mathrm{~S} 14 \mathrm{E} 25 \mathrm{~J} 01\end{array}$ & $\begin{array}{l}15 S 14 E 23 D 02 \\
16 S 16 E 03 E 02 \\
16 S 16 E 18 N 01 \\
16 S 16 E 20 N 01\end{array}$ & $\begin{array}{l}\text { 17S17E27R01 } \\
17 \mathrm{~S} 17 \mathrm{E} 35 \mathrm{E} 01 \\
17 \mathrm{~S} 18 \mathrm{E} 31 \mathrm{~N} 02 \\
18 \mathrm{~S} 18 \mathrm{E} 07 \mathrm{R} 01\end{array}$ \\
\hline $\begin{array}{l}13 \mathrm{~S} 15 \mathrm{E} 31 \mathrm{~J} 05 \\
14 \mathrm{~S} 14 \mathrm{E} 02 \mathrm{CO} 2 \\
14 \mathrm{S14E16N01}\end{array}$ & $\begin{array}{l}16 S 16 E 28 M 01 \\
17 S 16 E 02 E 01 \\
17 S 17 E 23 H 02\end{array}$ & $\begin{array}{l}\text { 18S18E07R02 } \\
18 \mathrm{~S} 18 \mathrm{E} 09 \mathrm{E} 01 \\
18 \mathrm{~S} 18 \mathrm{E} 09 \mathrm{~N} 01\end{array}$ \\
\hline
\end{tabular}

TABLE 5.--Number of wells perforated in the semiconfined zone at depths greater than 50 feet with perforated lengths in the Coast Range alluvium and Sierran sand

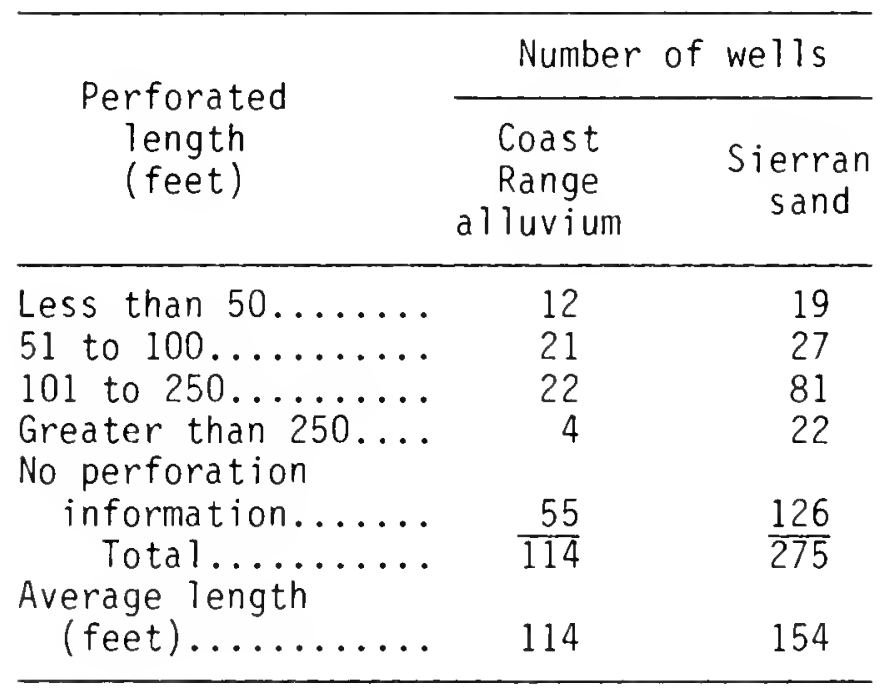

Wells perforated in the Sierran sand are primarily in the valley trough, where the Sierran sand is at least 200 feet thick. There are 275 wells perforated in the Sierran sand. The average perforated length of these wells is 154 feet. Most of the wells have perforated lengths more than 100 feet (table 2). The large number and relatively long perforated length of these wells reflects the high transmissivity of the Sierran sand.

The concentration of wells in the semiconfined zone perforated in the Sierran sand and the longer perforated length of wells tapping the Sierran sand compared to wells tapping the Coast Range alluvium indicate a tendency to use the Sierran sand rather than the Coast Range alluvium as a source of water.

\section{Wells Perforated in the Semiconfined and Confined Zones}

There are 533 wells perforated in the semiconfined and confined zones. The wells are grouped by the perforated length above and below the Corcoran Clay Member where Sierran sand is present and absent (table 6). The wells are presented by perforated length above the Corcoran in figure 9 and below the Corcoran in figure 10; these wells are listed in table 7 . 
TABLE 6.--Number of wells perforated in the semiconfined and confined zones with perforated lengths above and below the Corcoran Clay Member of the Tulare Formation where Sierran sand is present and absent

\begin{tabular}{|c|c|c|c|c|}
\hline \multirow{2}{*}{$\begin{array}{c}\text { Perforated } \\
\text { length below } \\
\text { the Corcoran } \\
\text { where Sierran } \\
\text { sand is present } \\
\text { (feet) }\end{array}$} & \multicolumn{4}{|c|}{$\begin{array}{c}\text { Perforated length above the } \\
\text { Corcoran where Sierran sand is } \\
\text { present (feet) }\end{array}$} \\
\hline & $\begin{array}{l}\text { Less than } \\
\text { or equal } \\
\text { to } 50\end{array}$ & $\begin{array}{c}51 \text { to } \\
100\end{array}$ & $\begin{array}{l}101 \text { to } \\
250\end{array}$ & $\begin{array}{l}251 \text { to } \\
500\end{array}$ \\
\hline \multicolumn{5}{|c|}{ Number of we $11 \mathrm{~s}$} \\
\hline $\begin{array}{l}\text { Less than or } \\
\text { equal to } 50 \ldots . \\
51 \text { to } 100 \ldots \ldots \\
101 \text { to } 500 \ldots \ldots \\
501 \text { to } 1,000 \ldots . \\
\text { Greater than } \\
1,000 \ldots . . . .\end{array}$ & $\begin{array}{r}0 \\
0 \\
8 \\
17\end{array}$ & $\begin{array}{r}0 \\
0 \\
9 \\
37\end{array}$ & $\begin{array}{r}4 \\
2 \\
41 \\
49\end{array}$ & $\begin{array}{r}9 \\
8 \\
22 \\
10\end{array}$ \\
\hline $\begin{array}{l}\text { Perforated } \\
\text { length below } \\
\text { the Corcoran }\end{array}$ & \multicolumn{4}{|c|}{$\begin{array}{l}\text { Perforated length above the } \\
\text { Corcoran where Sierran sand is } \\
\text { absent (feet) }\end{array}$} \\
\hline $\begin{array}{c}\text { where Sierran } \\
\text { sand is absent } \\
\text { (feet) }\end{array}$ & $\begin{array}{l}\text { Less than } \\
\text { or equal } \\
\text { to } 50\end{array}$ & $\begin{array}{c}51 \text { to } \\
100\end{array}$ & $\begin{array}{l}101 \\
250\end{array}$ to & $\begin{array}{l}251 \text { to } \\
500\end{array}$ \\
\hline \multicolumn{5}{|c|}{ Number of wells } \\
\hline $\begin{array}{l}\text { Less than or } \\
\text { equal to } 50 \ldots \ldots \\
51 \text { to } 100 \ldots \ldots \ldots \\
101 \text { to } 500 \ldots \ldots \\
501 \text { to } 1,000 \ldots \ldots \\
\text { Creater than } \\
1,000, \ldots \ldots \ldots\end{array}$ & $\begin{array}{r}0 \\
0 \\
3 \\
23\end{array}$ & $\begin{array}{r}0 \\
0 \\
7 \\
22\end{array}$ & $\begin{array}{r}1 \\
0 \\
9 \\
40\end{array}$ & $\begin{array}{r}3 \\
0 \\
10 \\
12\end{array}$ \\
\hline
\end{tabular}

Most of the wells have perforated lengths less than 250 feet above the Corcoran and all have perforated lengths less than 500 feet above the Corcoran. In contrast, most of the wells (397) have perforated lengths greater than 500 feet below the Corcoran. The distribution of perforated length reflects the tendency to use the confined system as the principal supply of water.

The degree to which the confined aquifer system is tapped as a source of supply depends, in part, on the presence or absence of Sierran sand above the Corcoran. There are 238 wells in or near the valley trough where the Sierran sand is present with a total perforated length of 38,527 feet above the Corcoran and 132,023 feet below the Corcoran. These wells have an average perforated length of 162 feet above the Corcoran and 555 feet below the Corcoran. In contrast, the 295 wells upslope of the valley trough, where the Sierran sand is absent, have a total perforated length of 39.034 feet above the Corcoran and 291,051 feet below the Corcoran. These wells have an average perforated length of 132 feet above the Corcoran and 987 feet below the Corcoran. The distribution of perforated length indicates that water supply wells need to be drilled deeper into the confined zone where the Sierran sand is absent than where it is present (table 6). 


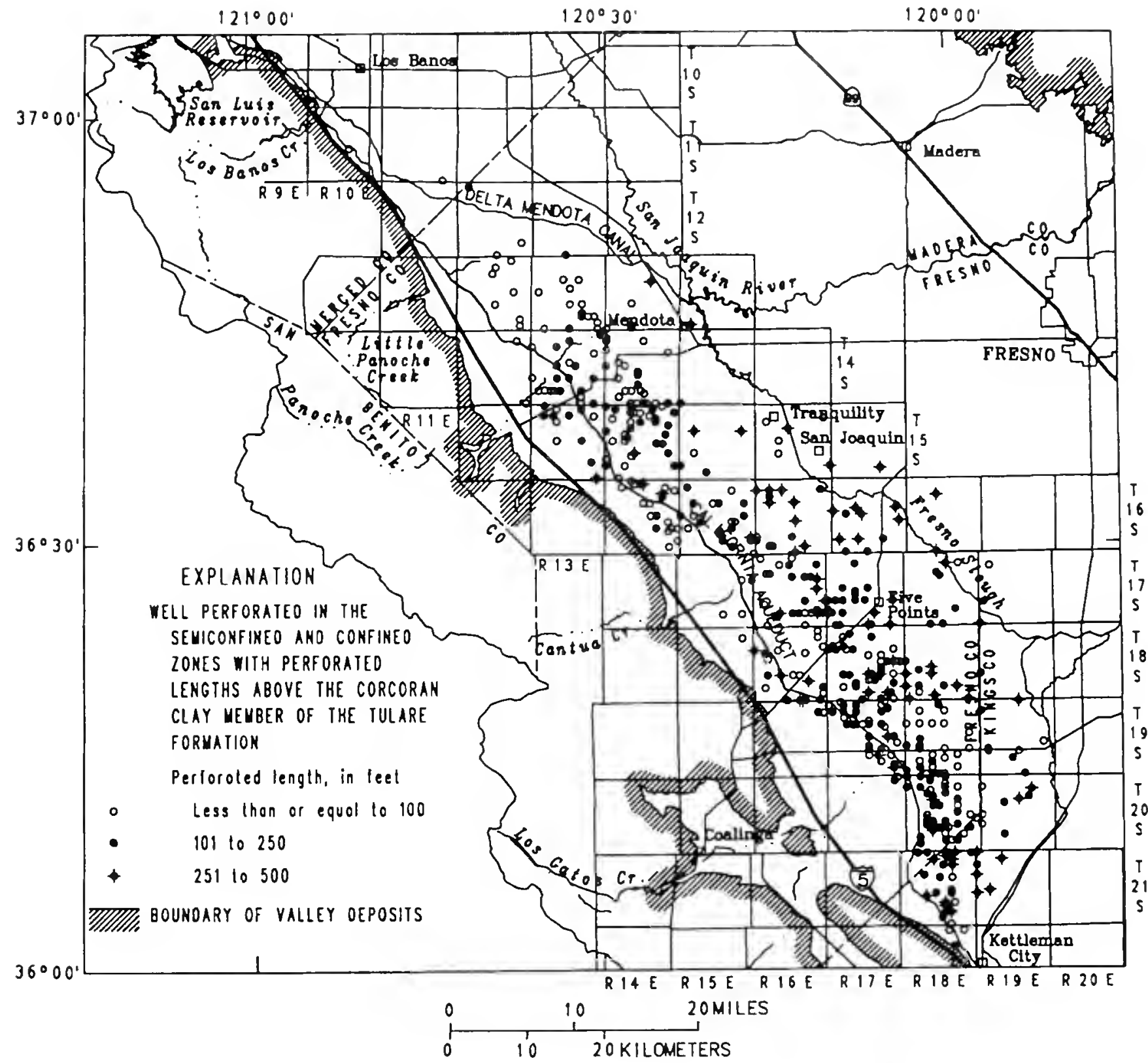

Figure 9. Areal distribution of wells perforated in the semiconfined and confined zones with perforated lengths above the Corcoron Cloy Member of the Tulare Formation. 


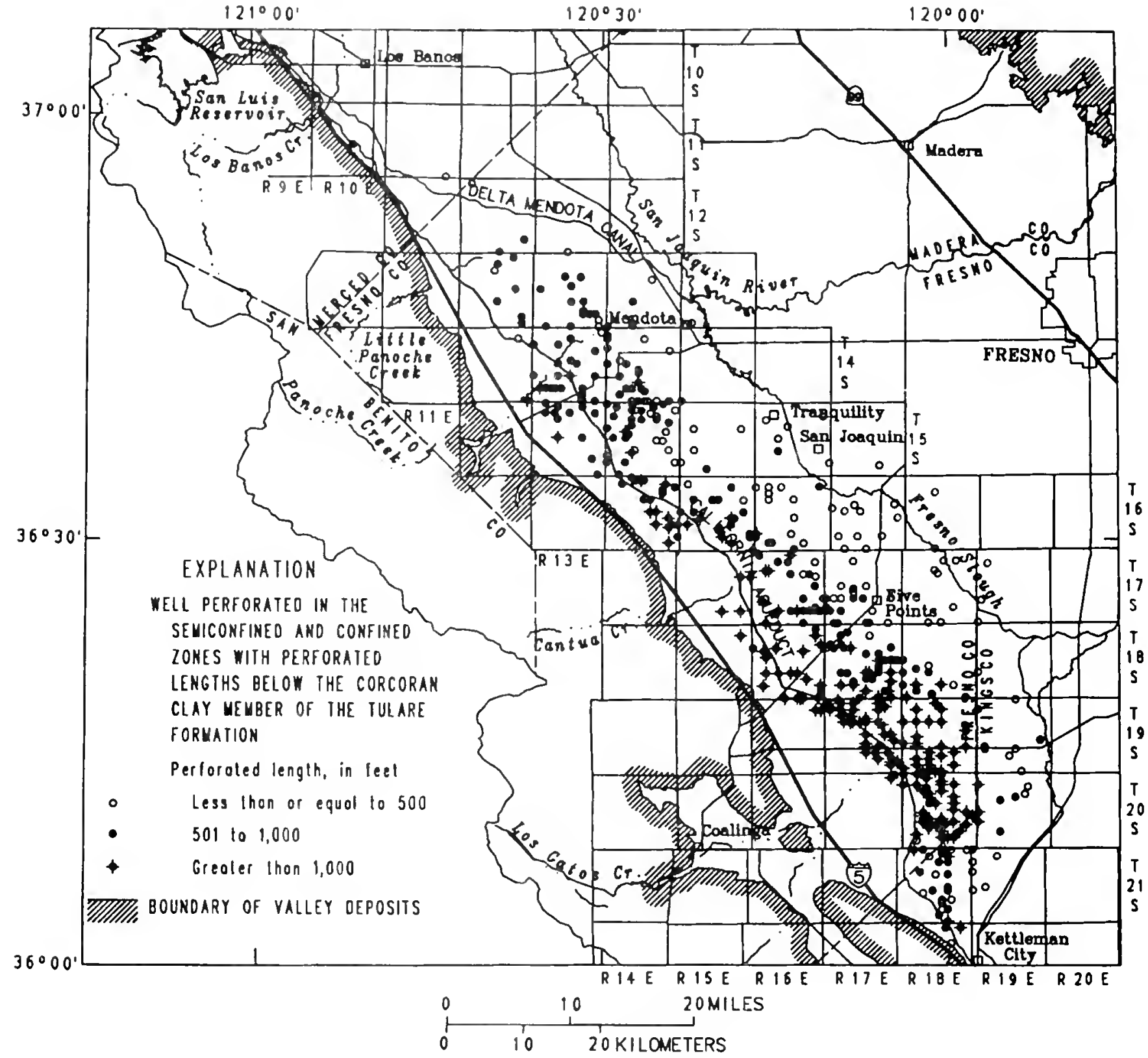

Figure 10. Areal distribution of wells perforated in the semiconfined and confined zones with perforated lengths below the Corcoran Clay Member of the Tulare

Formation. 
TABLE 7.--Wells perforated in the semiconfined and confined zones with perforated lengths above and below the Corcoron Clay Hember of the rulare Formation

\begin{tabular}{|c|c|c|c|c|c|}
\hline $\begin{array}{l}11 S 10 E 22 F 01 \\
11 S 11 E 3 S R 01 \\
12 S 12 E 06 H 01 \\
12 S 12 E 2 S N 01\end{array}$ & $\begin{array}{l}14 S 13 E 29001 \\
14 S 13 E 29 R 01 \\
14 S 13 E 30001 \\
14 S 13 E 35 E 01\end{array}$ & $\begin{array}{l}15 \mathrm{~S} 14 \mathrm{E} 06001 \\
1 \mathrm{SS} 14 \mathrm{E} 06 \mathrm{HO} 1 \\
15514 \mathrm{E} 06 \mathrm{HO} 2 \\
1 \mathrm{SS} 14 \mathrm{E} 07 \mathrm{BO} 1\end{array}$ & 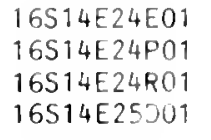 & $\begin{array}{l}\text { 16S17E32N01 } \\
16 S 17 E 33 R 01 \\
16 S 18 E 09 F 01 \\
16 S 18 E 33 P 01\end{array}$ & $\begin{array}{l}\text { 17S17E24P01 } \\
\text { 17S17E27001 } \\
\text { 17S17E27002 } \\
\text { 17S17E29N01 }\end{array}$ \\
\hline $\begin{array}{l}12 \mathrm{~S} 12 \mathrm{E} 34 \mathrm{~N} 01 \\
12 \mathrm{~S} 13 \mathrm{E} 33001 \\
13 \mathrm{~S} 12 \mathrm{E} 02 \mathrm{~F} 01 \\
13 \mathrm{~S} 12 \mathrm{E} 03 \mathrm{NO} 1\end{array}$ & $\begin{array}{l}14 S 13 E 35 N 01 \\
14 S 13 E 36 N 01 \\
14 S 14 E 03 N 02 \\
14 S 14 E 06 M 01\end{array}$ & $\begin{array}{l}15 S 14 \mathrm{E} 09 \mathrm{EO} 1 \\
1 \mathrm{SS14E09H01} \\
15514 \mathrm{E} 09 \mathrm{NO} 1 \\
15 S 14 \mathrm{E} 11001\end{array}$ & $\begin{array}{l}\text { 16S14E2SR01 } \\
16 S 14 \mathrm{E} 36 \mathrm{E} 01 \\
16 S 15 \mathrm{E} 02 \mathrm{NO} 1 \\
16 \mathrm{~S} 15 \mathrm{E} 06 \mathrm{KO} 01\end{array}$ & $\begin{array}{l}\text { 16S18E33P02 } \\
17 \mathrm{~S} 15 \mathrm{SE} 13 \mathrm{AO} 1 \\
17 \mathrm{~S} 15 \mathrm{E} 13001 \\
17 \mathrm{~S} 1 \mathrm{SE} 25 \mathrm{HO} 1\end{array}$ & $\begin{array}{l}\text { 17S17E29NO2 } \\
\text { 17S17E3OHO1 } \\
17 \text { S17E3ONO1 } \\
17517 \text { E3OPO2 }\end{array}$ \\
\hline $\begin{array}{l}13 \mathrm{~S} 12 \mathrm{E} 09 \mathrm{HO} 02 \\
13 \mathrm{~S} 12 \mathrm{E} 14001 \\
13 \mathrm{~S} 12 \mathrm{E} 22 \mathrm{NO} 1 \\
13 \mathrm{~S} 12 \mathrm{E} 36002\end{array}$ & $\begin{array}{l}14 \mathrm{~S} 14 \mathrm{E} 06 \mathrm{NO} 02 \\
14 \mathrm{S14E07MO1} \\
14 \mathrm{~S} 14 \mathrm{E} 12 \mathrm{NO} 2 \\
14 \mathrm{~S} 14 \mathrm{E} 17 \mathrm{MO} 1\end{array}$ & $\begin{array}{l}\text { 1SS14E11E02 } \\
15 S 14 \mathrm{E} 12 \mathrm{MO} 01 \\
1 \mathrm{SS} 14 \mathrm{E} 14 \mathrm{HO} 1 \\
15 \mathrm{~S} 14 \mathrm{E} 17 \mathrm{CO} 1\end{array}$ & $\begin{array}{l}\text { 16S1SE08N01 } \\
16 \text { S 1 SE09001 } \\
16 \text { S 15E1 ON02 } \\
\text { 16S1SE10N04 }\end{array}$ & $\begin{array}{l}17 S 15 E 27001 \\
17516 E 01 \mathrm{NO} 2 \\
17516 \mathrm{EO} 3 \mathrm{EO} 1 \\
17516 \mathrm{E} 04 \mathrm{NO} 2\end{array}$ & $\begin{array}{l}17 S 17 E 31001 \\
17 S 17 E 31 \mathrm{RO} 1 \\
17517 \mathrm{E} 34 \mathrm{NO} 1 \\
17517 \mathrm{E} 34 \mathrm{PO} 1\end{array}$ \\
\hline $\begin{array}{l}\text { 13S12E36MO1 } \\
13 S 13 \text { EO9Q01 } \\
13 S 13 E 10 R 01 \\
13 S 13 E 1 \text { SMO1 }\end{array}$ & $\begin{array}{l}14 S 14 E 17001 \\
14 S 14 E 17003 \\
14 S 14 E 18 \mathrm{NO} 1 \\
14 S 14 \mathrm{E} 20 \mathrm{NO} 1\end{array}$ & $\begin{array}{l}\text { 15S14E17J01 } \\
1 \text { SS14E17001 } \\
1 \mathrm{SS} 14 \mathrm{E} 18001 \\
1 \mathrm{SS} 14 \mathrm{E} 18001\end{array}$ & $\begin{array}{l}\text { 16S1SE1 2N01 } \\
\text { 16S1SE13NO1 } \\
\text { 16S1SE17N01 } \\
16 \text { S1SE18N01 }\end{array}$ & $\begin{array}{l}17 \mathrm{~S} 16 \mathrm{E} 04 \mathrm{RO} 2 \\
17 \mathrm{~S} 16 \mathrm{E} 06 \mathrm{CO} 1 \\
17 \mathrm{~S} 16 \mathrm{E} 08 \mathrm{LO} 1 \\
17 \mathrm{~S} 16 \mathrm{EOSMO2}\end{array}$ & $\begin{array}{l}\text { 17S17E35R01 } \\
17 S 18 E 02 P 01 \\
17 S 18 E 03 P 01 \\
17 S 18 E 09 K 01\end{array}$ \\
\hline $\begin{array}{l}13 S 13 E 18001 \\
13 S 13 E 22 N 01 \\
13 S 13 E 23 N 01 \\
13 S 13 E 2 S N 01\end{array}$ & $\begin{array}{l}14 \mathrm{S14E} 21 \mathrm{CO} 2 \\
14 \mathrm{S1} 14 \mathrm{E} 21 \mathrm{KO} 1 \\
14 \mathrm{S14E2} 8 \mathrm{CO} 1 \\
14 \mathrm{S14E} 28 \mathrm{NO} 1\end{array}$ & 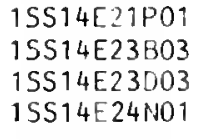 & $\begin{array}{l}16 S 1 S E 19001 \\
1651 S E 20 \mathrm{CO} 01 \\
16 S 15 E 23 E 03 \\
16 S 15 E 23 F 01\end{array}$ & $\begin{array}{l}17 S 16 E 10+101 \\
17 S 16 E 11 \mathrm{NO} 3 \\
17 \mathrm{~S} 16 \mathrm{E} 11001 \\
17 \mathrm{~S} 16 \mathrm{E} 13001\end{array}$ & $\begin{array}{l}\text { 17S18E09R01 } \\
17 \mathrm{~S} 18 \mathrm{E} 26 \mathrm{M} 101 \\
17 \mathrm{~S} 18 \mathrm{E} 3 \mathrm{NO} 1 \\
17 \mathrm{~S} 18 \mathrm{E} 34 \mathrm{NO} 1\end{array}$ \\
\hline $\begin{array}{l}13 S 13 E 2 S N 02 \\
13 S 13 E 26 M 01 \\
13 S 13 E 26 N 02 \\
13513 E 26001\end{array}$ & $\begin{array}{l}14 \mathrm{~S} 14 \mathrm{E} 28 \mathrm{R} 01 \\
14 \mathrm{S14E2} 2 \mathrm{Q} 01 \\
14 \mathrm{~S} 14 \mathrm{E} 32 \mathrm{EO} 2 \\
14 \mathrm{~S} 14 \mathrm{E} 33 \mathrm{NO} 1\end{array}$ & $\begin{array}{l}1 S S 14 E 2 S 001 \\
15 S 14 E 28 D 01 \\
1 S S 14 E 29001 \\
1 S S 14 E 30 E 01\end{array}$ & $\begin{array}{l}16 S 15 E 23 N 02 \\
16 S 1 S E 25 K 01 \\
16 S 15 E 25002 \\
16 S 1 S E 27001\end{array}$ & $\begin{array}{l}17 \mathrm{~S} 16 \mathrm{E} 13 \mathrm{~N} 01 \\
17 \mathrm{~S} 16 \mathrm{E} 13 \mathrm{NO} 2 \\
17 \mathrm{~S} 16 \mathrm{E} 23 \mathrm{~N} 02 \\
17 \mathrm{~S} 16 \mathrm{E} 24 \mathrm{R} 01\end{array}$ & $\begin{array}{l}\text { 17S19E07A01 } \\
17 \text { S19E30C01 } \\
17 \text { S19E31NO1 } \\
\text { 18S1SE01NO1 }\end{array}$ \\
\hline $\begin{array}{l}13 \mathrm{~S} 13 \mathrm{E} 32 \mathrm{NO} 2 \\
13 \mathrm{~S} 13 \mathrm{E} 34 \mathrm{NO} 4 \\
13 \mathrm{~S} 13 \mathrm{E} 36 \mathrm{FO} 01 \\
13 \mathrm{~S} 13 \mathrm{E} 36 \mathrm{PO} 2\end{array}$ & $\begin{array}{l}14 \mathrm{~S} 14 \mathrm{E} 33001 \\
14 \mathrm{~S} 14 \mathrm{E} 34001 \\
14 \mathrm{~S} 14 \mathrm{E} 34 \mathrm{NO} 1 \\
14 \mathrm{~S} 14 \mathrm{E} 34 \mathrm{PO} 1\end{array}$ & $\begin{array}{l}\text { 1SS14E3ONO1 } \\
\text { 1SS14E32NO2 } \\
\text { ISS1SE14A01 } \\
\text { ISS1SE17DO1 }\end{array}$ & $\begin{array}{l}\text { 16S1SE27E01 } \\
16 S 15 E 27 P 01 \\
16 S 15 E 35 E 01 \\
16 S 15 E 36 R 01\end{array}$ & $\begin{array}{l}17 \mathrm{~S} 16 \mathrm{E} 2 \mathrm{SM} 01 \\
17 \mathrm{~S} 16 \mathrm{E} 2 \mathrm{SNO} 1 \\
17 \mathrm{~S} 16 \mathrm{E} 25 \mathrm{NO} 2 \\
17 \mathrm{~S} 16 \mathrm{E} 2 \mathrm{SPO} 1\end{array}$ & $\begin{array}{l}\text { 18S16E01E01 } \\
18516 E 01 \mathrm{NO} 1 \\
18516 \mathrm{E} 07 \mathrm{NO} 1 \\
18516 \mathrm{E} 08 \mathrm{RO} 1\end{array}$ \\
\hline $\begin{array}{l}13514 E 15 B 01 \\
13 S 14 E 21 N 01 \\
13514 E 31 N 01 \\
13514 E 33 M 01\end{array}$ & $\begin{array}{l}14 \text { S14E3SNO1 } \\
14 \text { S14E36NO1 } \\
14 \text { S1SE3iNO1 } \\
1 \text { SS13E01NO1 }\end{array}$ & $\begin{array}{l}\text { 1SS1SE2ON02 } \\
1 \text { SS1SE3ONO2 } \\
1 \text { SS15E33E01 } \\
15 S 16 E 07 H 01\end{array}$ & $\begin{array}{l}16 \mathrm{~S} 16 \mathrm{E} 01 \mathrm{~N} 01 \\
16 \mathrm{~S} 16 \mathrm{E} 04 \mathrm{~N} 01 \\
16 \mathrm{~S} 16 \mathrm{E} 0 \mathrm{SN} 01 \\
16 \mathrm{~S} 16 \mathrm{E} 06 \mathrm{~N} 01\end{array}$ & $\begin{array}{l}17 \mathrm{~S} 16 \mathrm{E} 25001 \\
17 \mathrm{~S} 16 \mathrm{E} 26 \mathrm{NO} 04 \\
17 \mathrm{~S} 16 \mathrm{E} 26002 \\
17 \mathrm{~S} 16 \mathrm{E} 27 \mathrm{NO} 1\end{array}$ & $\begin{array}{l}\text { 18S16E12KO1 } \\
18 \mathrm{~S} 16 \mathrm{E} 14 \mathrm{DO} 1 \\
18 \mathrm{~S} 16 \mathrm{E} 17 \mathrm{MO} 1 \\
18 \mathrm{~S} 16 \mathrm{E} 18 \mathrm{AO} 2\end{array}$ \\
\hline $\begin{array}{l}\text { 13S14E33N01 } \\
13 S 1 \text { SE31 J06 } \\
14 \text { S12E02R02 } \\
14 \text { S12E36001 }\end{array}$ & $\begin{array}{l}15 S 13 E 02 E 01 \\
1 S S 13 E 03 N O 1 \\
15 S 13 E 04 E 01 \\
1 S 513 E 0 S D 01\end{array}$ & $\begin{array}{l}15 S 16 E 09001 \\
15 S 16 E 12 C 03 \\
15 S 16 E 17 R 01 \\
15 S 16 E 2 C R 01\end{array}$ & $\begin{array}{l}16 \mathrm{~S} 16 \mathrm{E} 08 \mathrm{E} 01 \\
16 \mathrm{~S} 16 \mathrm{E} 08 \mathrm{~N} 01 \\
16 \mathrm{~S} 16 \mathrm{E} 10 \mathrm{~N} 01 \\
16 \mathrm{~S} 16 \mathrm{E} 22 \mathrm{E} 01\end{array}$ & $\begin{array}{l}17 S 16 E 27001 \\
17516 E 28 N 01 \\
17516 E 30 A 01 \\
17516 E 32 A 01\end{array}$ & $\begin{array}{l}\text { 18S16E20NO2 } \\
18 \mathrm{~S} 16 \mathrm{E} 21 \mathrm{NO} 1 \\
18 \mathrm{~S} 16 \mathrm{E} 22 \mathrm{NO} 1 \\
18 \mathrm{~S} 16 \mathrm{E} 22001\end{array}$ \\
\hline $\begin{array}{l}14 \mathrm{~S} 13 \mathrm{EO} 1 \mathrm{CO} 1 \\
14 \mathrm{~S} 13 \mathrm{E} 01 \mathrm{CO} 2 \\
14 \mathrm{~S} 13 \mathrm{E} 03 \mathrm{NO} 1 \\
14 \mathrm{~S} 13 \mathrm{E} 07 \mathrm{E} 02\end{array}$ & $\begin{array}{l}\text { 1SS13EOSNO1 } \\
1 \text { SS13EOSR01 } \\
15 S 13 E 09 E 01 \\
1 S S 13 E 14 \text { NO1 }\end{array}$ & $\begin{array}{l}\text { 15S16E 2SR01 } \\
15 S 17 E 34 A 01 \\
16 S 14 E 01002 \\
16 S 14 E 02 J 01\end{array}$ & $\begin{array}{l}\text { 16S16E28J01 } \\
16516 E 30 M 01 \\
16516 E 30 N 01 \\
16516 E 32 E 02\end{array}$ & $\begin{array}{l}17516 E 32 \mathrm{NO} 1 \\
17516 \mathrm{E} 35 \mathrm{NO} 1 \\
17517 \mathrm{E} 04 \mathrm{~N} 01 \\
1751 / \mathrm{S} 1 \mathrm{SN} 01\end{array}$ & $\begin{array}{l}\text { 18S16E2SO01 } \\
18 \mathrm{~S} 16 \mathrm{E} 26 \mathrm{~F} 01 \\
18 \mathrm{~S} 16 \mathrm{E} 26 \mathrm{~F} 02 \\
18 \mathrm{~S} 16 \mathrm{E} 30 \mathrm{R} 01\end{array}$ \\
\hline $\begin{array}{l}14 \text { S13E09E01 } \\
14 \text { S13E12NO1 } \\
14 \text { S13E1SM01 } \\
14 \text { S13E1SO01 }\end{array}$ & $\begin{array}{l}\text { 1SS13E16N01 } \\
15 S 13 E 26 J 01 \\
15 S 13 E 36 P 01 \\
15 S 13 E 36 P 02\end{array}$ & $\begin{array}{l}16 \mathrm{~S} 14 \mathrm{E} 03 \mathrm{EO} 1 \\
16 \mathrm{~S} 14 \mathrm{E} 04 \mathrm{DO} 01 \\
16 \mathrm{~S} 14 \mathrm{E} 04 \mathrm{FO} 1 \\
16 \mathrm{~S} 14 \mathrm{E} 04 \mathrm{HO} 1\end{array}$ & $\begin{array}{l}16516 \mathrm{E} 32 \mathrm{FO} 1 \\
16 \mathrm{S1} 16 \mathrm{E} 34 \mathrm{HO} 1 \\
16516 \mathrm{E} 34 \mathrm{NO} 2 \\
1651 ? \mathrm{E} 13 \mathrm{EO} 1\end{array}$ & $\begin{array}{l}17517 E 15 N 02 \\
17 S 17 E 17 N 01 \\
17 S 17 E 17 N 02 \\
17 S 17 E 19 N 01\end{array}$ & $\begin{array}{l}\text { 18S16E } 33001 \\
18 S 16 E 34001 \\
18 S 16 E 34 R 01 \\
18 S 16 E 35 N 01\end{array}$ \\
\hline $\begin{array}{l}\text { 14S13E16N01 } \\
14 \text { S13E19N01 } \\
14 \text { S13E21N01 } \\
14 \text { S13E22N01 }\end{array}$ & $\begin{array}{l}1 \mathrm{SS} 14 \mathrm{E} 01 \mathrm{~K} 01 \\
15 \mathrm{~S} 14 \mathrm{EO} 2 \mathrm{BO} 1 \\
1 \mathrm{SS} 14 \mathrm{E} 03 \mathrm{HO} 1 \\
1 \mathrm{SS} 14 \mathrm{E} 03001\end{array}$ & $\begin{array}{l}\text { 16S14EOSLO1 } \\
16 S 14 \text { E10NO1 } \\
16 S 14 \text { E10Q01 } \\
16 S 14 E 11 \mathrm{BO} 1\end{array}$ & $\begin{array}{l}16517 \mathrm{E} 16 \mathrm{PO} 1 \\
16517 \mathrm{E} 17 \mathrm{H} 01 \\
16517 \mathrm{E} 17 \mathrm{~N} 01 \\
16517 \mathrm{E} 18 \mathrm{M} 01\end{array}$ & $\begin{array}{l}\text { 17S17E19N02 } \\
17 \text { S17E19001 } \\
17 \text { S17E20N01 } \\
17 \text { S17E21001 }\end{array}$ & $\begin{array}{l}\text { 18S16E36C01 } \\
18 S 16 E 36 P 01 \\
18 S 17 E 03 P 01 \\
18 S 17 E 0 S K 01\end{array}$ \\
\hline $\begin{array}{l}\text { 14S13E24N01 } \\
14 \text { S13E26N01 } \\
\text { 14S13E28P01 } \\
14 \text { S13E29N01 }\end{array}$ & $\begin{array}{l}\text { 15S14EO4D01 } \\
\text { 1SS14EO4J01 } \\
\text { 15S14EO4LO1 } \\
15 S 14 E 04 M 01\end{array}$ & $\begin{array}{l}16 S 14 \mathrm{E} 11 \mathrm{CO} 2 \\
16 \mathrm{~S} 14 \mathrm{E} 14 \mathrm{NO} 1 \\
16 \mathrm{~S} 14 \mathrm{E} 15001 \\
16514 \mathrm{E} 23 \mathrm{NO} 1\end{array}$ & $\begin{array}{l}16 S 17 E 24 F 01 \\
16 S 17 E 26 N 01 \\
16 S 17 E 28 N 01 \\
16517 E 30001\end{array}$ & $\begin{array}{l}17 S 17 E 21 E 01 \\
17 S 17 E 21 \mathrm{NO} 1 \\
17517 \mathrm{E} 21 \mathrm{NO} 2 \\
17 \mathrm{~S} 17 \mathrm{E} 21801\end{array}$ & $\begin{array}{l}\text { 18S17EOSK03 } \\
\text { 18S17E0SN03 } \\
\text { 18S17E07L01 } \\
\text { 18S17E07L02 }\end{array}$ \\
\hline
\end{tabular}


TABLE 7.--Wells perforated in the semiconfined and confined zones with perforoted lengths above and below the Corcoron Clay Member of the Tulore Formation--Continued

\begin{tabular}{|c|c|c|c|c|c|}
\hline 18S17E07N01 & 18S17E36N03 & 19S17E10N01 & 19S18E29N01 & 20S18E16E01 & $21 \mathrm{~S} 18 \mathrm{E} 02 \mathrm{CO} 1$ \\
\hline 18517E07001 & 18S18E18NO1 & 19S17E11N01 & 19S18E31M01 & 20S18E16G01 & 21S18E02M01 \\
\hline 18S17E08M01 & 18S18E19N01 & 19S17E13NO1 & 19S18E32E01 & 20S18E16CO2 & $21 \mathrm{~S} 18 \mathrm{E} 02 \mathrm{MO} 2$ \\
\hline 18S17E08P01 & 18S18E19N02 & 19S17E14C01 & 19S18E32001 & 20S18E20B01 & $21 \mathrm{~S} 18 \mathrm{E} 03 \mathrm{~A} 01$ \\
\hline 18S17E08P02 & $18 S 18 \mathrm{E} 20 \mathrm{M} 01$ & 19S17E14001 & 19S18E33E02 & 20S18E 20C01 & 21S18E03B01 \\
\hline 18S17E08R01 & 18S18E21E01 & 19S17E15N01 & 19S18E33N02 & 20S18E20K01 & $21 \mathrm{~S} 18 \mathrm{E} 03 \mathrm{CO} 1$ \\
\hline 18S17E12N01 & 18S18E21N01 & 19S17E22M01 & 19S18E 33N03 & 20S18E20001 & $21 \mathrm{~S} 18 \mathrm{E} 04 \mathrm{DO} 2$ \\
\hline 18S17E13N01 & 18 S18E26N01 & 19S17E22NO1 & 19S18E33001 & 20S18E21M01 & 21S18E04K01 \\
\hline 18S17E13N02 & 18S18E27N01 & 19S17E23N01 & 19S18E34N01 & 20S18E21N01 & $21 \mathrm{~S} 18 \mathrm{E} 05 \mathrm{CO} 1$ \\
\hline 18S17E13P01 & 18S18E29N01 & 19S17E24F01 & 19S18E35E01 & 20S18E 22M01 & 21S18E08R01 \\
\hline 18S17E13001 & 18S18E30N01 & 19S17E26H01 & 19S19E03001 & 20S18E23N01 & 21S18E10C01 \\
\hline 18S17E14E01 & 18S18E31P01 & 19S17E26N01 & 19S19E06N01 & 20S18E 24D01 & 21S18E11D01 \\
\hline 18S17E14N02 & $18 \mathrm{~S} 18 \mathrm{E} 32 \mathrm{E} 01$ & 19S17E27E01 & 19S19E 19001 & 20S18E24C01 & 21S18E14D01 \\
\hline 18S17E14Q01 & 18S18E33L01 & 19S17E27H01 & 19S19E24E01 & 20S18E25001 & 21S18E15D01 \\
\hline 18S17E15E01 & 18S18E36N02 & 19S17E36B01 & 19S19E27D01 & 20S18E25002 & $21 \mathrm{S18E21 \textrm {HO } 1}$ \\
\hline 18S17E20N.02 & 18S19E19N01 & $19517 \mathrm{E} 36002$ & 19S19E28K01 & 20S18E26K01 & 21S18E21M01 \\
\hline 18S17E20Q01 & 19S16E01N01 & 19S17E36E01 & 19S19E30B02 & 20S18E 27CO2 & 21S18E23D06 \\
\hline 18S17E22N02 & 19S 16E01001 & 19S18E03N02 & 19S19E34001 & 20S18E27001 & 21S18E23E01 \\
\hline 18S17E22P01 & 19S17E02C01 & $19 \mathrm{~S} 18 \mathrm{E} 04 \mathrm{CO} 2$ & 20S17E01C01 & 20S18E27M01 & 21S18E26C01 \\
\hline 18S17E23E01 & 19S17E02E01 & 19S18E05N01 & 20S17E01E01 & 20S18E28B01 & 21S18E26N01 \\
\hline 18S17E23M01 & 19S17E02K01 & 19S18E07N01 & $20 \mathrm{~S} 18 \mathrm{E} 02 \mathrm{NO} 2$ & $20 S 18 E 28001$ & 21S18E27B01 \\
\hline 18S17E23N01 & 19S17E02N01 & 19S18E08N01 & 20S18E03D01 & 20S18E28E01 & 21S18E27G01 \\
\hline 18S17E23N02 & 19S17E04E01 & 19S18E09N02 & $20 S 18 E 03 M 01$ & 20S18E29N01 & 21S18E27002 \\
\hline 18S17E24N02 & 19S17E04NO2 & 19S18E10N01 & 20S18E03NOI & 20S18E29NO2 & $21 S 18 E 34 F 01$ \\
\hline 18S17E25M01 & 19S17E05E01 & 19S18E13M01 & 20S18E05D01 & 20S18E32E01 & 21S18E35N01 \\
\hline 18S17E27E01 & 19S17E05N01 & 19S18E19M01 & 20S18E05H01 & 20S18E34B01 & 21S19E04M01 \\
\hline 18S17E27C01 & 19S17E05N03 & 19S18E20D01 & 20S18E05NO1 & 20S18E34001 & 21S19E06001 \\
\hline 18S17E28M01 & 19S17E06A01 & 19S18E20D02 & 20S18E06001 & 20S18E35001 & 21S19E06D02 \\
\hline 18S17E28N01 & 19S17E06E01 & 19S18E20M01 & 20S18E06N01 & 20S19E06́N01 & 21S19E06D03 \\
\hline 18S17E28N02 & 19S17E06P01 & 19S18E20N01 & 20S18E08A01 & 20S19E10J01 & 21S19E07001 \\
\hline 18S17E29N02 & 19S17E08E02 & 19S18E22N01 & 20S18E08C01 & 20S19E10N01 & 21S19E07N01 \\
\hline 18S17E33001 & 19S17E08H01 & 19S18E24N01 & 20S18E09M01 & 20S19E11001 & 21S19E19E02 \\
\hline 18S17E34E01 & 19S17E08P01 & 19S18E26E02 & 20S18E09N01 & $20 S 19 E 17 A 01$ & 21S19E20001 \\
\hline 18S17E34E02 & 19S17E08R01 & 19S18E27M01 & 20S18E10E01 & 20S19E19001 & 22S18E01E01 \\
\hline 18S17E35G01 & 19S17E09N01 & 19S18E28E01 & 20S18E11N01 & 20S19E19N01 & $22 \mathrm{~S} 18 \mathrm{E} 03 \mathrm{H} 01$ \\
\hline 18S17E35H01 & 19S17E09001 & 19S18E28N01 & 20S18E14E01 & 20S19E29J01 & 22S18E11L01 \\
\hline 18S17E35N01 & 19S17E10M01 & 19S18E28N02 & 20S18E14N01 & 21S18E01B01 & \\
\hline
\end{tabular}


Wells Perforated Only in the

\section{Confined Zone}

There are 410 wells perforated only in the confined zone below the Corcoran Clay Member of the Tulare Formation. Table 8 summarizes these wells by the relative distance between the bottom of the Corcoran and the top of the perforated length where the Sierran sand is present. For most of these wells, the top of the perforated length starts within the first 100 feet below the Corcoran. These wells also have perforated lengths ranging from 100 to more than 1,000 feet. All wells are presented in figure 11 grouped by perforated length. This figure shows that, in general, the perforated length increases in a southwest direction upslope toward the Coast Ranges. Table 9 lists the wells perforated only in the confined zone.

Wells perforated only in the confined zone have a shorter average perforated length in areas where the Sierran sand is present (628 feet) than in areas where the Sierran sand is absent 1987 feet). There are 113 wells in the valley trough, where the Sierran sand is present, with a total perforated length of 70,985 feet. There are 297 wells upslope of the valley trough with a total perforated length of 293,038 feet.

Most of the wells are upslope of the valley trough. Wells are highly concentrated near the creeks in the fanhead areas, as compared to the interfan areas. In addition, this indicates a tendency for locating wells in the coarser grained sediments of the upper-fan areas rather than in the finer grained sediments of the interfan areas.
TABLE 8.--Number of wells perfarated only in the confined zone with perforated lengths below the Corcoran Clay Member of the Tulore Formation where the Sierron sand is present and absent

\begin{tabular}{|c|c|c|c|c|c|c|}
\hline \multirow{2}{*}{$\begin{array}{l}\text { Perfor- } \\
\text { ated } \\
\text { length } \\
\text { (feet) }\end{array}$} & \multicolumn{6}{|c|}{$\begin{array}{c}\text { Distance between bottom of the Corcoran } \\
\text { and top of perforated length where the } \\
\text { Sierran sand is present (feet) }\end{array}$} \\
\hline & $\begin{array}{c}\ln \\
\text { Corcoran }\end{array}$ & $\begin{array}{l}\text { Less } \\
\text { than } \\
50\end{array}$ & $\begin{array}{l}51 \\
\text { to } \\
100\end{array}$ & $\begin{array}{l}101 \\
\text { to } \\
500\end{array}$ & $\begin{array}{l}501 \\
\text { to } \\
1,000\end{array}$ & $\begin{array}{l}\text { Greater } \\
\text { than } \\
1,000\end{array}$ \\
\hline \multicolumn{7}{|l|}{ Less than } \\
\hline $\begin{array}{l}\text { or equal } \\
\text { to } 50 . . \\
51 \text { to }\end{array}$ & 0 & 1 & 1 & 3 & 0 & 0 \\
\hline $\begin{array}{c}100 \ldots \\
101 \text { to }\end{array}$ & 1 & 0 & 1 & 1 & 0 & 0 \\
\hline $500 \ldots$ & 11 & 12 & 5 & 8 & 0 & 0 \\
\hline $\begin{array}{l}1,000 \ldots \\
\text { Creater }\end{array}$ & 23 & 14 & 4 & 4 & 0 & 0 \\
\hline $\begin{array}{l}\text { than } \\
1,000 \ldots\end{array}$ & 10 & 6 & 4 & 4 & 0 & 0 \\
\hline \multirow{2}{*}{$\begin{array}{l}\text { Perfor- } \\
\text { ated } \\
\text { length } \\
\text { (feet) }\end{array}$} & \multicolumn{6}{|c|}{$\begin{array}{c}\text { Distance between bottom of the Corcoran } \\
\text { and top of perforated length where the } \\
\text { Sierran sand is absent (feet) }\end{array}$} \\
\hline & $\begin{array}{c}\text { In } \\
\text { Corcoran }\end{array}$ & $\begin{array}{l}\text { Less } \\
\text { than } \\
50\end{array}$ & $\begin{array}{l}51 \\
\text { to } \\
100\end{array}$ & $\begin{array}{l}101 \\
\text { to } \\
500\end{array}$ & $\begin{array}{c}501 \\
\text { to } \\
1,000\end{array}$ & $\begin{array}{c}\text { Greater } \\
\text { than } \\
1,000\end{array}$ \\
\hline \multicolumn{7}{|l|}{ Less than } \\
\hline 51 to $50 \ldots$ & 0 & 2 & 0 & 0 & 0 & 1 \\
\hline $100 \ldots$ & 0 & 3 & 0 & 2 & 0 & 0 \\
\hline $\begin{array}{l}500 \ldots \\
501 \text { to }\end{array}$ & 6 & 14 & 5 & 9 & 1 & 0 \\
\hline $\begin{array}{l}1,000 \ldots \\
\text { Creater }\end{array}$ & 44 & 38 & 16 & 2 & 0 & 2 \\
\hline $1,000 \ldots$ & 38 & 31 & 33 & 40 & 7 & 3 \\
\hline
\end{tabular}




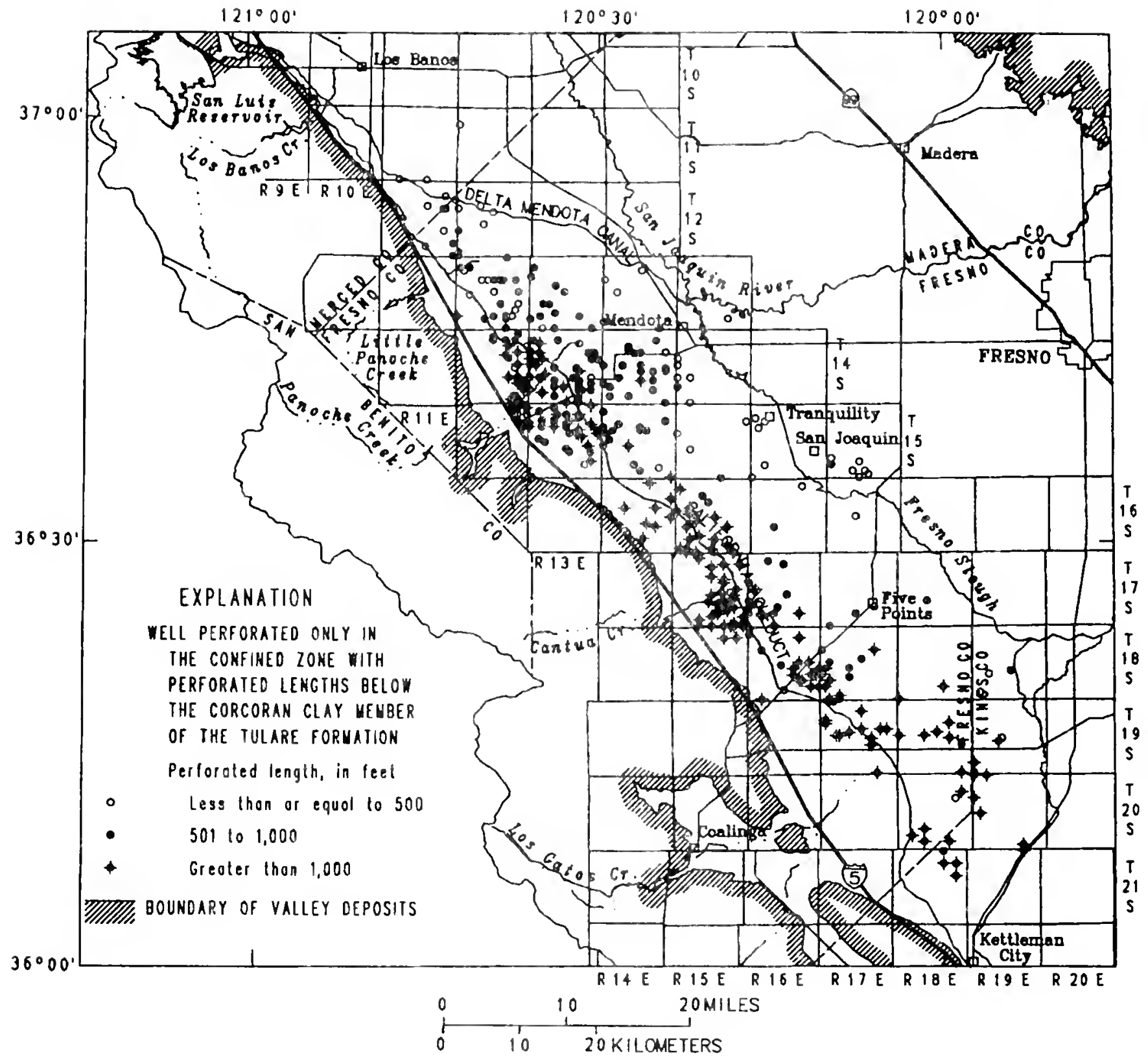

Figure 11. Areal distribution of wells perforated only in the confined zone with perforated lengths below the Corcoran Clay Nember of the Tulare Formation. 
TABLE 9.--Wells perforated only in the confined zone with perforated lengths below the Corcoran Clay Member of the Tulare Formation

\begin{tabular}{|c|c|c|c|c|c|}
\hline $\begin{array}{l}11 S 11 E 32 P 01 \\
11 S 11 E 34 Q 01 \\
11 S 12 E 07 E 02 \\
12 S 11 E 10 Q 01\end{array}$ & $\begin{array}{l}\text { 13S13E2ON01 } \\
13 S 13 E 20001 \\
13 S 13 E 26 N 03 \\
13 S 13 E 28 M 01\end{array}$ & $\begin{array}{l}14 \mathrm{~S} 13 \mathrm{E} 13 \mathrm{E} 02 \\
14 \mathrm{~S} 13 \mathrm{E} 13 \mathrm{CO} 1 \\
14 \mathrm{~S} 13 \mathrm{E} 17 \mathrm{E} 01 \\
14 \mathrm{~S} 13 \mathrm{E} 18 \mathrm{E} 01\end{array}$ & $\begin{array}{l}14 S 14 E 30 E 03 \\
14 S 14 E 30 K 01 \\
14514 E 31 \mathrm{G02} \\
14514 E 33 E 02\end{array}$ & $\begin{array}{l}\text { 15S14E06M01 } \\
15 S 14 \text { E07N01 } \\
15 S 14 \text { E08LO1 } \\
\text { 15S14E1ONO1 }\end{array}$ & $\begin{array}{l}\text { 16S15E27N02 } \\
\text { 16S15E29J02 } \\
\text { 16S15E29N01 } \\
\text { 16S15E31 J01 }\end{array}$ \\
\hline $\begin{array}{l}\text { 12S11E12D01 } \\
12 S 11 E 12 \mathrm{HO1} \\
12 \mathrm{~S} 11 \mathrm{E} 13002 \\
12 S 11 \mathrm{E} 14 \mathrm{AO} 1\end{array}$ & $\begin{array}{l}\text { 13S13E30R01 } \\
13513 E 31001 \\
13 S 13 E 32 N 03 \\
13 S 13 E 33 N 02\end{array}$ & $\begin{array}{l}14 S 13 E 18 \mathrm{NO} 1 \\
14513 \mathrm{E} 18 \mathrm{NO} 2 \\
14 \mathrm{~S} 13 \mathrm{E} 18001 \\
14 \mathrm{~S} 13 \mathrm{E} 19 \mathrm{NO} 2\end{array}$ & $\begin{array}{l}\text { 14S14E36N02 } \\
14515 E 18 E 01 \\
14515 E 18 E 02 \\
14515 E 18 N 01\end{array}$ & $\begin{array}{l}\text { 15S14E12E01 } \\
15 S 14 E 15801 \\
15 S 14 E 15 D 01 \\
15 S 14 E 18 A 01\end{array}$ & $\begin{array}{l}\text { 16S15E31L01 } \\
\text { 16S15E31R01 } \\
\text { 16S15E32001 } \\
\text { 16S15E33J01 }\end{array}$ \\
\hline $\begin{array}{l}12 S 11 E 17002 \\
12 S 11 E 23 R 02 \\
12 S 11 E 2 S 001 \\
12 S 11 E 34 C 03\end{array}$ & $\begin{array}{l}\text { 13S13E36N01 } \\
13 \mathrm{~S} 14 \mathrm{E} 07 \mathrm{NO} 1 \\
13 \mathrm{~S} 14 \mathrm{E} 10001 \\
13 \mathrm{~S} 14 \mathrm{E} 17 \mathrm{NO} 04\end{array}$ & $\begin{array}{l}\text { 14S13E19R01 } \\
\text { 14S13E20LO1 } \\
\text { 14S13E21D01 } \\
14513 E 22 A 01\end{array}$ & $\begin{array}{l}\text { 14S1SE19N01 } \\
\text { 14S15E2ONO1 } \\
\text { 14S15E3OM01 } \\
14 \text { S15E32N02 }\end{array}$ & $\begin{array}{l}15 S 14 E 18 F 01 \\
15 S 14 E 18002 \\
15 S 14 E 19 E 01 \\
15 S 14 E 21 E 01\end{array}$ & $\begin{array}{l}\text { 16S1SE34N04 } \\
16 S 1 S E 3 S E 02 \\
16 S 1 S E 35002 \\
16 S 16 E 02 M 01\end{array}$ \\
\hline $\begin{array}{l}12 \mathrm{~S} 11 \mathrm{E} 36001 \\
12 \mathrm{~S} 12 \mathrm{E} 08 \mathrm{R} 01 \\
12 \mathrm{~S} 12 \mathrm{E} 16 \mathrm{HOS} \\
12 \mathrm{~S} 12 \mathrm{E} 16 \mathrm{HO} 6\end{array}$ & $\begin{array}{l}13 S 14 E 31 E 01 \\
13 S 14 E 35002 \\
13 S 15 E 35003 \\
14 S 12 E 01 E 01\end{array}$ & $\begin{array}{l}14 S 13 E 22001 \\
14513 E 23 E 01 \\
14 S 13 E 24 N 04 \\
14 S 13 E 2 S E 01\end{array}$ & $\begin{array}{l}\text { 15512E01B01 } \\
\text { 15512E01E01 } \\
\text { 1SS12E01H01 } \\
\text { 15S12E01N02 }\end{array}$ & $\begin{array}{l}15 S 14 E 28001 \\
15514 E 34 E 01 \\
15 S 14 E 35801 \\
15514 E 36002\end{array}$ & $\begin{array}{l}\text { 16S16E2OR01 } \\
16 S 17 E 21 \mathrm{CO} 1 \\
17 \mathrm{~S} 15 \mathrm{~S} 01 \mathrm{PO} 1 \\
17 \mathrm{~S} 15 \mathrm{~S} 02 \mathrm{~N} 01\end{array}$ \\
\hline $\begin{array}{l}12 S 12 E 18001 \\
12 S 12 E 19 N 01 \\
12 S 12 E 21 E 01 \\
12 S 12 E 31 M 01\end{array}$ & $\begin{array}{l}\text { 14S12E02N01 } \\
14 \mathrm{~S} 12 \mathrm{E} 02 \mathrm{NO} 2 \\
14 \mathrm{~S} 12 \mathrm{E} 02 \mathrm{R0} 1 \\
14 \mathrm{~S} 12 \mathrm{E} 03 \mathrm{M} 01\end{array}$ & $\begin{array}{l}14 S 13 E 25 N 01 \\
14 S 13 E 26 D 01 \\
14 S 13 E 26 E 02 \\
14513 E 261101\end{array}$ & $\begin{array}{l}\text { 15S12E01R01 } \\
\text { 15S12E02G01 } \\
15 S 12 \mathrm{E} 12 \mathrm{E} 01 \\
15512 \mathrm{E} 12 \mathrm{Q} 01\end{array}$ & $\begin{array}{l}\text { 15S1SE12G01 } \\
1 \text { S515E18E02 } \\
15 S 15 E 20 M 01 \\
15 S 15 E 34001\end{array}$ & $\begin{array}{l}\text { 17S15E04E01 } \\
17 \text { S15E05A01 } \\
17 \text { S1SE } 10001 \\
17 \text { S1SE10N01 }\end{array}$ \\
\hline $\begin{array}{l}12 S 12 E 31 N 01 \\
12 S 12 E 31 N 02 \\
13 S 12 E 02 D 01 \\
13 S 12 E 0 S N 01\end{array}$ & $\begin{array}{l}\text { 14512E03N01 } \\
\text { 14S12E1 IF01 } \\
\text { 14S12E11F02 } \\
14 \mathrm{~S} 12 \mathrm{E} 12 \mathrm{HO} 1\end{array}$ & $\begin{array}{l}14 S 13 E 26 M 02 \\
14 S 13 E 26 \mathrm{NO} 2 \\
14 S 13 E 26 \mathrm{PO} 1 \\
14 S 13 E 28 D 01\end{array}$ & $\begin{array}{l}15 S 12 \mathrm{E} 12002 \\
15 \mathrm{~S} 13 \mathrm{E} 01 \mathrm{DO} 1 \\
1 \mathrm{SS} 13 \mathrm{E} 02 \mathrm{NO} 2 \\
15 \mathrm{~S} 13 \mathrm{E} 03 \mathrm{NO} 2\end{array}$ & $\begin{array}{l}\text { 15S16E07C01 } \\
15 S 16 E 07002 \\
1 \text { SS16E08E01 } \\
15516 E 29 N 01\end{array}$ & $\begin{array}{l}\text { 17S15E13N01 } \\
17 S 15 E 14 D 01 \\
17 S 15 E 14 E 01 \\
17 S 15 E 21 R 01\end{array}$ \\
\hline $\begin{array}{l}\text { 13S12E06P01 } \\
13 S 12 E 07 B 01 \\
13 S 12 E 09 N 01 \\
13 S 12 E 09001\end{array}$ & $\begin{array}{l}\text { 14S12E12N01 } \\
\text { 14S12E13N01 } \\
14 \mathrm{~S} 12 \mathrm{E} 14001 \\
14 \mathrm{~S} 12 \mathrm{E} 14 \mathrm{~F} 01\end{array}$ & $\begin{array}{l}\text { 14S13E28J01 } \\
14513 \text { E28M01 } \\
14 \text { S13E30N01 } \\
14 \text { S13E32001 }\end{array}$ & $\begin{array}{l}\text { 15S13E04E02 } \\
15 S 13 E 04 P 01 \\
15 S 13 E 06 J 01 \\
15 S 13 E 07 M 01\end{array}$ & $\begin{array}{l}\text { 15S16E31N02 } \\
15517 E 28 \mathrm{KO} 1 \\
15517 E 30 \mathrm{FO} 1 \\
15517 E 30 \mathrm{PO} 1\end{array}$ & $\begin{array}{l}\text { 17S15E22A01 } \\
17515 E 22 E 01 \\
17 S 15 E 23 H 01 \\
17 S 15 E 23 J 01\end{array}$ \\
\hline $\begin{array}{l}\text { 13S12E1ONO1 } \\
13 S 12 E 10 Q 01 \\
13 S 12 E 10 R 01 \\
13 S 12 E 11 R 01\end{array}$ & $\begin{array}{l}14 \mathrm{~S} 12 \mathrm{E} 23 \mathrm{AO} 1 \\
14 \mathrm{~S} 12 \mathrm{E} 23 \mathrm{PO} 1 \\
14 \mathrm{~S} 12 \mathrm{E} 24 \mathrm{NO} 1 \\
14 \mathrm{~S} 12 \mathrm{E} 25 \mathrm{AO} 1\end{array}$ & $\begin{array}{l}14 S 13 E 33 E 01 \\
14513 E 34 N 01 \\
14 S 13 E 35 E 02 \\
14 S 13 E 35 N 02\end{array}$ & $\begin{array}{l}\text { 15S13E07N01 } \\
\text { 15S13E08E01 } \\
15 \mathrm{~S} 13 E 08 N 02 \\
15 S 13 E 09 E 02\end{array}$ & $\begin{array}{l}\text { 15S17E33E01 } \\
15 S 17 E 33001 \\
15 S 17 E 34 E 01 \\
15 S 17 E 34 L 01\end{array}$ & $\begin{array}{l}\text { 17S15E23N02 } \\
17 \text { S15E26C01 } \\
17 \text { S15E26H01 } \\
17515 E 26 L 01\end{array}$ \\
\hline $\begin{array}{l}13 S 12 E 13 N 01 \\
13 S 12 E 15 N 01 \\
13 S 12 E 20001 \\
13 S 12 E 22001\end{array}$ & $\begin{array}{l}14 \mathrm{~S} 12 \mathrm{E} 25001 \\
14 \mathrm{~S} 12 \mathrm{E} 2 \mathrm{SE} 01 \\
14 \mathrm{~S} 12 \mathrm{E} 25001 \\
14 \mathrm{~S} 12 \mathrm{E} 25002\end{array}$ & $\begin{array}{l}\text { 14514E02P01 } \\
14 \text { S14E09E02 } \\
14 \text { S14E09E04 } \\
14514 \text { E09M01 }\end{array}$ & $\begin{array}{l}\text { 15S13EO9N02 } \\
15 S 13 E 10 F 01 \\
15513 E 10 M 01 \\
15513 E 10 N 01\end{array}$ & $\begin{array}{l}\text { 15S17E34LO2 } \\
\text { 16S14E03HO1 } \\
16 S 14 E 12 \mathrm{KO} 1 \\
16 \mathrm{~S} 14 \mathrm{E} 14 \mathrm{FO} 1\end{array}$ & $\begin{array}{l}\text { 17S1SE27801 } \\
17 S 15 E 27 K 01 \\
17 S 15 E 27002 \\
17 S 15 E 33 E 02\end{array}$ \\
\hline $\begin{array}{l}13 \mathrm{~S} 12 \mathrm{E} 24 \mathrm{NO} 1 \\
13 \mathrm{~S} 12 \mathrm{E} 24 \mathrm{NO} 2 \\
13 \mathrm{~S} 12 \mathrm{E} 26 \mathrm{HO} 1 \\
13 \mathrm{~S} 12 \mathrm{E} 26 \mathrm{NO} 1\end{array}$ & $\begin{array}{l}\text { 14S12E3SH01 } \\
\text { 14S12E35J01 } \\
\text { 14S12E35K01 } \\
14 \text { S12E35001 }\end{array}$ & $\begin{array}{l}\text { 14S14E09001 } \\
14 \text { S14E1ONO1 } \\
14 \text { S14E1ONO4 } \\
14 \text { S14E12NO1 }\end{array}$ & $\begin{array}{l}\text { 15S13E11D02 } \\
15513 \mathrm{E} 12 \mathrm{M} 01 \\
15513 \mathrm{E} 13 \mathrm{~B} 01 \\
15513 \mathrm{E} 13 \mathrm{GO} 1\end{array}$ & $\begin{array}{l}16 \mathrm{~S} 14 \mathrm{E} 17 \mathrm{HO} 1 \\
16514 \mathrm{E} 22 \mathrm{KO} 1 \\
16514 \mathrm{E} 23 \mathrm{PO} 01 \\
16 \mathrm{~S} 14 \mathrm{E} 24 \mathrm{BO} 1\end{array}$ & $\begin{array}{l}\text { 17S15E33N01 } \\
17 \text { S15E34D01 } \\
\text { 17S15E34N01 } \\
17515 E 35 J 01\end{array}$ \\
\hline $\begin{array}{l}13 \mathrm{~S} 12 \mathrm{E} 26001 \\
13 \mathrm{~S} 12 \mathrm{E} 27 \mathrm{NO} 1 \\
13 \mathrm{~S} 12 \mathrm{E} 30 \mathrm{NO} 1 \\
13 \mathrm{~S} 12 \mathrm{E} 3 \mathrm{C} 01\end{array}$ & $\begin{array}{l}\text { 14S12E36M01 } \\
\text { 14S13E04P01 } \\
\text { 14S13E06P01 } \\
\text { 14S13E06P02 }\end{array}$ & $\begin{array}{l}\text { 14S14E15N01 } \\
14 \mathrm{~S} 14 \mathrm{E} 17 \mathrm{Q} 02 \\
14 \mathrm{~S} 14 \mathrm{E} 18 \mathrm{NO} 2 \\
14514 \mathrm{E} 20 \mathrm{PO} 1\end{array}$ & $\begin{array}{l}\text { 15S13E14001 } \\
\text { 15S13E14L01 } \\
\text { 15S13E14M01 } \\
15 S 13 E 15 F 01\end{array}$ & $\begin{array}{l}\text { 16S14E27P01 } \\
16 S 15 E 06 P 01 \\
16 S 15 E 09 E 01 \\
16 S 15 E 09002\end{array}$ & $\begin{array}{l}\text { 17S1SE3SN01 } \\
17 \text { S1SE3S001 } \\
17 S 15 E 35 R 01 \\
17 S 15 E 36801\end{array}$ \\
\hline $\begin{array}{l}13 S 12 E 35001 \\
13 S 12 E 3 S N 01 \\
13 S 12 E 35 N 02 \\
13 S 13 E 06 P 01\end{array}$ & $\begin{array}{l}\text { 14S13E07A01 } \\
\text { 14S13E07N02 } \\
\text { 14S13E07R01 } \\
14 S 13 E 11004\end{array}$ & $\begin{array}{l}\text { 14S14E22E01 } \\
14 \text { S14E23E01 } \\
14 \text { S14E24D01 } \\
14514 E 24 E 01\end{array}$ & $\begin{array}{l}\text { 15S13E18R01 } \\
15 \mathrm{~S} 13 \mathrm{E} 20 \mathrm{CO} 1 \\
15 \mathrm{~S} 13 \mathrm{E} 22 \mathrm{MO} 1 \\
15513 \mathrm{E} 22 \mathrm{PO} 1\end{array}$ & $\begin{array}{l}\text { 16S15E17E01 } \\
16 \text { S15E17M01 } \\
16 \text { S1SE17N02 } \\
16 S 1 S E 18 M 01\end{array}$ & $\begin{array}{l}\text { 17S15E36K01 } \\
17 S 15 E 36001 \\
17 S 15 E 36002 \\
17 S 16 E 04 R 01\end{array}$ \\
\hline $\begin{array}{l}\text { 13S13E06P02 } \\
13 S 13 E 07 M 01 \\
13 S 13 E 16 E 02 \\
13 S 13 E 16 R 01\end{array}$ & $\begin{array}{l}\text { 14S13E11006 } \\
14 \mathrm{~S} 13 \mathrm{E} 11 \mathrm{RO} 1 \\
14 \mathrm{~S} 13 \mathrm{E} 12 \mathrm{NO} 2 \\
14 \mathrm{~S} 13 \mathrm{E} 12 \mathrm{PO} 1\end{array}$ & $\begin{array}{l}14 S 14 E 24 E 02 \\
14 S 14 E 26001 \\
14 S 14 E 26 N 01 \\
14 S 14 E 27001\end{array}$ & $\begin{array}{l}\text { 15S13E23H01 } \\
15 S 13 E 24 N 01 \\
15 S 13 E 25 M 01 \\
15 S 13 E 26002\end{array}$ & $\begin{array}{l}\text { 16S15E19R01 } \\
16 S 1 \text { SE20C03 } \\
16 S 15 E 22001 \\
16 S 15 E 23 N 01\end{array}$ & $\begin{array}{l}\text { 17S16E05K01 } \\
17 \mathrm{~S} 16 \mathrm{E} 18 \mathrm{E} 01 \\
17 \mathrm{~S} 16 \mathrm{E} 18002 \\
17516 \mathrm{E} 19 \mathrm{~N} 01\end{array}$ \\
\hline
\end{tabular}


TABLE 9.--Wells perforated only in the confined zone with perforated lengths below the Corcoran Clay Member of the Tulare Formation--Continued

\begin{tabular}{|c|c|c|c|c|c|}
\hline $\begin{array}{l}\text { 17S16E22E01 } \\
17 \mathrm{~S} 16 \mathrm{E} 26 \mathrm{NO} 1 \\
17 \mathrm{~S} 16 \mathrm{E} 28 \mathrm{~F} 01 \\
17 \mathrm{~S} 16 \mathrm{E} 28 \mathrm{NO} 2\end{array}$ & $\begin{array}{l}\text { 18S16E02N01 } \\
18 \mathrm{~S} 16 \mathrm{E} 06001 \\
18 \mathrm{~S} 16 \mathrm{E} 06 \mathrm{M0} 1 \\
18 \mathrm{~S} 16 \mathrm{E} 08 \mathrm{NO} 1\end{array}$ & $\begin{array}{l}\text { 18S16E26F03 } \\
\text { 18S16E32N01 } \\
\text { 18S16E33A01 } \\
\text { 18S17E07NO4 }\end{array}$ & $\begin{array}{l}\text { 18S18E31NO2 } \\
\text { 18S19E20P01 } \\
\text { 18S19E22MO2 } \\
\text { 18S19E31C01 }\end{array}$ & $\begin{array}{l}\text { 19S17E3SP01 } \\
\text { 19S18E11N01 } \\
\text { 19S18E1SM01 } \\
\text { 19S18E16N02 }\end{array}$ & $\begin{array}{l}20 S 18 E 33 E 02 \\
20 S 18 E 3 S E 01 \\
20 S 19 E 0 S 001 \\
20 S 19 E 06001\end{array}$ \\
\hline $\begin{array}{l}\text { 17S16E29N01 } \\
17 S 16 E 30 A 0 S \\
17 S 16 E 30 A 06 \\
17 S 16 E 30 E 01\end{array}$ & $\begin{array}{l}\text { 18S16E14NO2 } \\
\text { 18S16E14RO2 } \\
\text { 18S16E21B01 } \\
\text { 18S16E22G01 }\end{array}$ & $\begin{array}{l}\text { 18S17E11N02 } \\
\text { 18S17E1SM01 } \\
\text { 18S17E18001 } \\
\text { :8S17E19F01 }\end{array}$ & $\begin{array}{l}\text { 18S19E31C02 } \\
\text { 19S17E03NO2 } \\
\text { 19S17E06A02 } \\
\text { 19S17E07LO1 }\end{array}$ & $\begin{array}{l}\text { 19S18E18N03 } \\
\text { 19S18E23002 } \\
\text { 19S18E24M01 } \\
\text { 19S18E36N01 }\end{array}$ & $\begin{array}{l}\text { 20S19E07N01 } \\
20 S 19 E 19 B 01 \\
20 S 19 E 3 S M 01 \\
21 S 18 E 02002\end{array}$ \\
\hline $\begin{array}{l}\text { 17S16E30N01 } \\
17 S 16 E 32 D 01 \\
17 S 16 E 33 N 01 \\
17 S 17 E 26 E 03\end{array}$ & $\begin{array}{l}\text { 18S16E 22002 } \\
18 \mathrm{~S} 16 \mathrm{E} 24 \mathrm{HO} 2 \\
18 \mathrm{~S} 16 \mathrm{E} 24 \mathrm{NO} 1 \\
18 \mathrm{~S} 16 \mathrm{E} 24 \mathrm{PO} 1\end{array}$ & $\begin{array}{l}\text { 18S17E19N01 } \\
\text { 18S17E21E01 } \\
\text { 18S17E28D01 } \\
\text { 18S17E30C01 }\end{array}$ & $\begin{array}{l}\text { 19S17E07P01 } \\
\text { 19S17E13E01 } \\
\text { 19S17E14C01 } \\
\text { 19S17E1SE01 }\end{array}$ & $\begin{array}{l}\text { 19S19E21C01 } \\
\text { 19S19E21E01 } \\
\text { 19S19E31001 } \\
\text { 19S19E31N01 }\end{array}$ & $\begin{array}{l}21 S 18 E 02 D 03 \\
21 S 18 E 11002 \\
21 S 18 E 12001 \\
21 S 18 E 13001\end{array}$ \\
\hline $\begin{array}{l}\text { 17S17E28001 } \\
17 S 17 E 31002 \\
17 S 18 E 21 P 01 \\
18 S 1 S E 01 N 02 \\
18 S 1 S E 02 N 02\end{array}$ & $\begin{array}{l}18 S 16 E 24001 \\
18 S 16 E 24002 \\
18 S 16 E 2 S N 01 \\
18 S 16 E 2 S R 01 \\
18 S 16 E 26 E 01\end{array}$ & $\begin{array}{l}\text { 18S17E30P02 } \\
\text { 18S17E31001 } \\
\text { 18S17E32L01 } \\
\text { 18S17E32P01 } \\
\text { 18S18E27001 }\end{array}$ & $\begin{array}{l}\text { 19S17E16M01 } \\
\text { 19S17E17NO2 } \\
\text { 19S17E17P01 } \\
\text { 19S17E22A01 } \\
\text { 19S17E22J03 }\end{array}$ & $\begin{array}{l}20 S 18 E 11002 \\
20 S 18 E 11003 \\
20 S 18 E 12 E 01 \\
20 S 18 E 28 E 02 \\
20 S 18 E 29 N 03\end{array}$ & 21S19E02B01 \\
\hline
\end{tabular}

\section{WELLS WITH WATER LEVELS}

\section{REPRESENTATIVE OF THE}

\section{SEMICONFINED ZONE}

A contour map of water-table altitude is helpful for characterizing the flow system of the semiconfined zone. The presence of a downward hydraulic head gradient in the study area necessitates care in selecting wells indicative of the water table. Well depths and 1984 water-level measurements were examined to determine which wells could be used to construct a water-table map. Most of these wells have water-level measurements recorded since 1967. In general, the shallowest wells available were used as indicators of the water table. Where the water table is less than 20 feet deep, 20-foot and shal- lower wells were used. Further upslope toward the Coast Ranges, where the water table is deeper, wells are more sparsely distributed. In these areas. most of the wells completed in the semiconfined zone were used. Where the Corcoran is not present, wells less than 1,000 feet deep were used to help define the water table. Dry wells also were helpful in determining the upper limit of the water table. Table 10 lists wells with water levels indicative of the water table and dry wells, which help define the water table. Figure 12 shows the areal distribution of these wells. Figure 13 shows the wells perforated below the water table and above the Corcoran Clay Member of the Tulare Formation. These wells are indicative of water levels in the semiconfined zone below the water table and are listed in table 11 . 


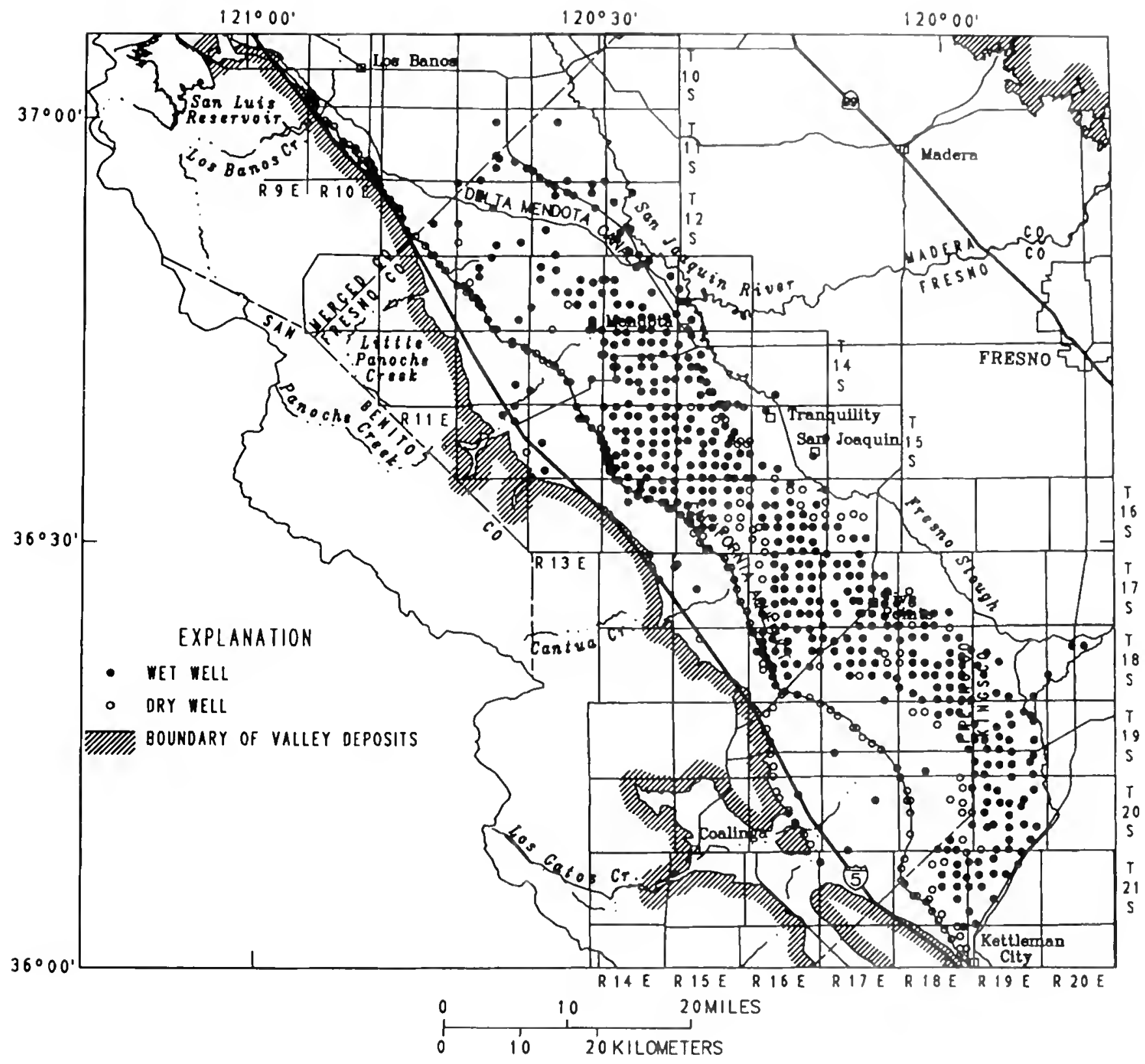

Figure 12. Areal distribution of wells with water levels indicative of the water table and dry wells which help define the water table. 


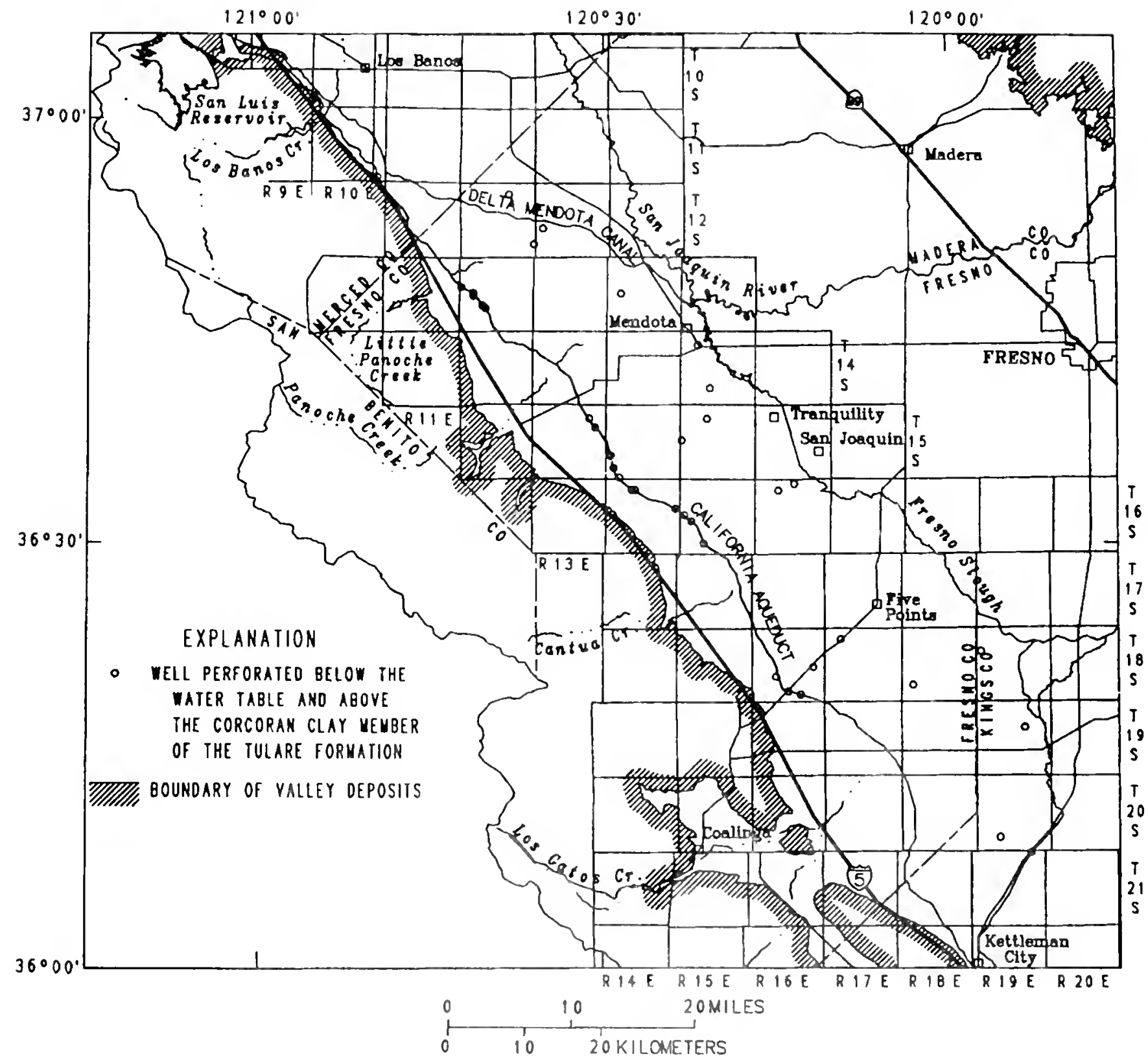

Figure 13. Areal distribution of wells perforated bclow the water table and above the Corcoran Clay Member of the Tulare Formation. 
TABLE 10.--Wells with water levels indicative of the water table and dry wells which help define the water table

$$
[* \text {, dry wel1s] }
$$

\begin{tabular}{|c|c|c|c|c|c|}
\hline $\begin{array}{r}10 S 10 E 31001 \\
10 S 10 E 31 \mathrm{CO} 2 \\
\star 10 S 10 \mathrm{E} 31001 \\
10 S 10 \mathrm{E} 31002\end{array}$ & $\begin{array}{r}\star 12 S 11 E 20 R 01 \\
\star 12 S 11 E 20 R 02 \\
12 S 11 E 23 E 01 \\
\star 12 S 11 E 28 C 01\end{array}$ & $\begin{array}{r}13 S 12 E 20 R 01 \\
13 S 12 E 28 D 01 \\
\star 13 S 12 E 33804 \\
\star 13 S 12 E 34 N 01\end{array}$ & $\begin{array}{r}\text { 13515E19M03 } \\
13 \text { S15E31R02 } \\
13 \text { S15E32D01 } \\
* 14 \text { S12E02Q01 }\end{array}$ & $\begin{array}{l}\text { 14S14E28A01 } \\
14 \text { S14E28D01 } \\
14 \text { S14E33NO2 } \\
14 \text { S15E0SE01 }\end{array}$ & $\begin{array}{l}\text { 1SS14E17A01 } \\
\text { 1SS14E17001 } \\
15 S 14 \mathrm{E} 17 \mathrm{R} 01 \\
15 S 14 \mathrm{E} 19001\end{array}$ \\
\hline $\begin{array}{l}* 11 \text { S10E08CO2 } \\
* 11 \text { S10EOBCO1 } \\
* 11 \text { S10E09M01 } \\
* 11 \text { S10E09M02 }\end{array}$ & $\begin{array}{l}\star 12 S 11 E 28 \mathrm{CO} 2 \\
\star 12 S 11 E 34 \mathrm{CO} 1 \\
\star 12 S 11 E 34 \mathrm{CO} 2 \\
\star 12 S 11 E 34 R 02\end{array}$ & $\begin{array}{l}13 S 12 E 34 P 02 \\
13 S 12 E 36 D 03 \\
13 S 13 E 06 R 01 \\
13 S 13 E 07 R 01\end{array}$ & $\begin{array}{r}\star 14 S 12 \mathrm{E} 11 \mathrm{BO} 1 \\
\star 14 \mathrm{~S} 12 \mathrm{E} 12 \mathrm{CO} 02 \\
14 \mathrm{~S} 12 \mathrm{E} 12 \mathrm{~J} 01 \\
14 \mathrm{S12E} 26 \mathrm{H} 01\end{array}$ & $\begin{array}{l}\text { 14S15E05E02 } \\
14 \text { S15E05E03 } \\
14 \text { S1SE07D01 } \\
14 \text { S } 15 E 08 R 01\end{array}$ & $\begin{array}{l}\text { 15514E19L01 } \\
\text { 15S14E19M01 } \\
\text { 15S14E20R01 } \\
\text { 1SS14E22001 }\end{array}$ \\
\hline $\begin{array}{l}\star 11 \text { S10EO9M03 } \\
\star 11 \text { S10E16R01 } \\
\star 11 \text { S10E16R02 } \\
\star 11 \text { S10E21A02 }\end{array}$ & $\begin{array}{r}\text { *12S11E3SN03 } \\
12 S 12 E 06001 \\
12 S 12 E 08 A 01 \\
12 S 12 E 16 A 01\end{array}$ & $\begin{array}{l}13 S 13 E 09002 \\
13 S 13 E 11 N 03 \\
13 S 13 E 12 R 02 \\
13 S 13 E 13001\end{array}$ & $\begin{array}{l}* 14 \text { S13E07001 } \\
\star 14 S 13 E 07002 \\
\star 14 S 13 E 12 R 01 \\
* 14 S 13 E 16 N 02\end{array}$ & $\begin{array}{l}\text { 14S1SE17N01 } \\
14 \text { S15E17Q02 } \\
\text { 14S1SE18A01 } \\
\text { 14S1SE18N02 }\end{array}$ & $\begin{array}{l}1 S S 14 E 23004 \\
1 S S 14 E 23 N 01 \\
1 S S 14 E 24002 \\
1 S S 14 E 25 D 01\end{array}$ \\
\hline $\begin{array}{l}* 11 \mathrm{~S} 10 \mathrm{E} 22 \mathrm{CO} 1 \\
* 11 \mathrm{~S} 10 \mathrm{E} 22 \mathrm{HO} \\
* 11 \mathrm{~S} 10 \mathrm{E} 22 \mathrm{J01} \\
* 11 \mathrm{S1OE} 23 \mathrm{NO} 01\end{array}$ & $\begin{array}{r}12 S 12 E 19 E 01 \\
12 S 12 E 2 S N 02 \\
\star 12 S 12 E 30 N 02 \\
12 S 12 E 34003\end{array}$ & $\begin{array}{l}13 S 13 E 13 R 02 \\
13 S 13 E 14 N 03 \\
13 S 13 E 1 S N O 2 \\
13 S 13 E 16 N 02\end{array}$ & $\begin{array}{l}\star 14 \text { S13E16N03 } \\
\star 14 S 13 E 16 N 04 \\
\star 14 S 13 E 17 E 03 \\
\star 14 S 13 E 17 K 01\end{array}$ & $\begin{array}{l}\text { 14S1SE19R02 } \\
14 \text { S1SE20R01 } \\
14 \text { S1SE21D01 } \\
14 \text { S15E28C01 }\end{array}$ & $\begin{array}{l}\text { 15S14E25N01 } \\
\text { 1SS14E28A01 } \\
\text { 1SS14E29D01 } \\
\text { 1SS14E29R02 }\end{array}$ \\
\hline $\begin{array}{r}11 \text { S10E2SM01 } \\
11 \text { S10E2SN01 } \\
\star 11 \text { S10E26F01 } \\
11 \text { S10E26H01 }\end{array}$ & $\begin{array}{l}12 S 13 E 02 A 01 \\
12 S 13 E 02 R 01 \\
12 S 13 E 04 R 06 \\
12 S 13 E 05 C 0 S\end{array}$ & $\begin{array}{r}* 13 S 13 E 22 N 02 \\
13 S 13 E 22 R 01 \\
13 S 13 E 24 N 01 \\
13 S 13 E 30001\end{array}$ & $\begin{array}{r}\star 14 S 13 E 17 K 02 \\
* 14 S 13 E 21 \mathrm{CO} 1 \\
* 14 \mathrm{~S} 13 \mathrm{E} 21 \mathrm{CO} 2 \\
14 \mathrm{~S} 13 \mathrm{E} 24 \mathrm{NO} 02\end{array}$ & $\begin{array}{l}14 \text { S15E28001 } \\
14 \text { SISE28R01 } \\
14 \text { S1SE32A02 } \\
14 \text { S1SE34P01 }\end{array}$ & $\begin{array}{l}\text { 1SS14E30C02 } \\
1 \mathrm{SS} 14 \mathrm{E} 30 \mathrm{KO} 02 \\
1 \mathrm{SS} 14 \mathrm{E} 30 \mathrm{LO} 1 \\
1 \mathrm{SS} 14 \mathrm{E} 31802\end{array}$ \\
\hline $\begin{array}{l}11 \text { S10E26J01 } \\
11 \text { S10E36C02 } \\
11 \text { S10E36D01 } \\
11 \text { S10E36E01 }\end{array}$ & $\begin{array}{l}12 S 13 \text { EOSCO6 } \\
12 S 13 \text { ESHOS } \\
12 S 13 E 0 S H 06 \\
12 S 13 E 08 A 05\end{array}$ & $\begin{array}{r}\text { *13S13E32R01 } \\
13 S 13 E 3 S R 01 \\
13 S 13 E 36001 \\
13513 E 36 E 01\end{array}$ & $\begin{array}{r}* 14 \text { S13E27C01 } \\
\star 14 \text { S13E27D01 } \\
* 14 \text { S13E3ONO2 } \\
14 \text { S13E3ONO4 }\end{array}$ & $\begin{array}{l}14 S 1 \text { SE34001 } \\
14 \text { S1SE3SNO2 } \\
14 \text { S1SE3SR02 } \\
14 \text { S16E32A03 }\end{array}$ & $\begin{array}{r}1 S S 14 E 31 \mathrm{CO} 1 \\
* 1 \mathrm{SS} 14 \mathrm{E} 31 \mathrm{HO} 4 \\
1 \mathrm{SS} 14 \mathrm{E} 32001 \\
\text { 1SS14E33N01 }\end{array}$ \\
\hline $\begin{array}{l}11 \text { S1OE36F01 } \\
11 \text { S1OE36L02 } \\
11 S 10 E 36 M 01 \\
11 S 10 E 36 Q 01\end{array}$ & $\begin{array}{l}12 S 13 E 10 \mathrm{COS} \\
12 \mathrm{~S} 13 \mathrm{E} 13002 \\
12 \mathrm{~S} 13 \mathrm{E} 20 \mathrm{NO} \\
12 \mathrm{~S} 13 \mathrm{E} 32 \mathrm{RO} 1\end{array}$ & $\begin{array}{l}\text { 13S13E36M02 } \\
13 S 13 E 36 \mathrm{NO} 4 \\
13 \mathrm{~S} 13 \mathrm{E} 36 \mathrm{R} 01 \\
13 \mathrm{~S} 14 \mathrm{E} 03 \mathrm{GO} 1\end{array}$ & $\begin{array}{l}14 S 14 \mathrm{E} 01004 \\
14 S 14 \mathrm{E} 02 \mathrm{RO} 1 \\
14 \mathrm{~S} 14 \mathrm{EO} \mathrm{NO} 04 \\
14 \mathrm{~S} 14 \mathrm{E} 03 \mathrm{RO} 1\end{array}$ & $\begin{array}{r}\text { 1SS12E02A01 } \\
1 \text { 1SS12E03R01 } \\
1 S S 12 E 11 \mathrm{HO1} \\
* 1 S S 12 \mathrm{E} 24 \mathrm{RO} 1\end{array}$ & $\begin{array}{l}\text { 1SS14E34A01 } \\
15 S 14 E 34001 \\
15 S 14 E 34001 \\
1 S S 14 E 36 A 01\end{array}$ \\
\hline $\begin{array}{l}11 S 12 E 10 D 01 \\
11 S 12 E 22 N 05 \\
11 S 12 E 2 S Q 0 S \\
11 S 12 E 25 Q 06\end{array}$ & $\begin{array}{l}\text { 12S14E04N01 } \\
12 S 14 E 07 K 01 \\
12 S 14 E 20 K 01 \\
12 S 14 E 20 R 01\end{array}$ & $\begin{array}{l}13 \mathrm{~S} 14 \mathrm{E} 03 \mathrm{LO} 1 \\
13 \mathrm{~S} 14 \mathrm{E} 03 \mathrm{MO} 2 \\
13 \mathrm{~S} 14 \mathrm{E} 03 \mathrm{MO4} \\
13 \mathrm{~S} 14 \mathrm{E} 04 \mathrm{QO} 1\end{array}$ & $\begin{array}{l}14 S 14 E 05 A 01 \\
14 S 14 E 05 A 03 \\
14 S 14 E 0 S 002 \\
14 S 14 E 0 S R 01\end{array}$ & $\begin{array}{r}\text { *1SS13E03A01 } \\
\text { 1SS13E11B01 } \\
\text { 1SS13E11C01 } \\
\text { 1SS13E11003 }\end{array}$ & $\begin{array}{l}\text { 1SS1SE01C01 } \\
1 \text { SS1SE03A01 } \\
\text { 1SS1SE03Q02 } \\
\text { 1SS1SE04A01 }\end{array}$ \\
\hline $\begin{array}{l}11 S 12 E 26 \mathrm{COS} \\
11 \mathrm{~S} 12 \mathrm{E} 26 \mathrm{CO} \\
11 \mathrm{~S} 12 \mathrm{E} 27 \mathrm{COS} \\
11 \mathrm{~S} 12 \mathrm{E} 27 \mathrm{NO} 1\end{array}$ & $\begin{array}{l}12 S 14 E 29002 \\
12 S 14 E 30 J 0 S \\
12 S 14 E 32 J 01 \\
12 S 14 E 32 J 03\end{array}$ & $\begin{array}{l}\text { 13S14E07N05 } \\
13 S 14 E 07 R 01 \\
13 S 14 E 12 L 01 \\
13 S 14 E 1 S R 01\end{array}$ & $\begin{array}{l}\text { 14S14E06N03 } \\
14 S 14 E 07 A 01 \\
14 S 14 E 07 N 02 \\
14 S 14 E 11 R 01\end{array}$ & $\begin{array}{r}\text { 1SS13E12NO3 } \\
\star 1 S S 13 E 12 R 01 \\
15 S 13 E 13 \mathrm{JO} 01 \\
\text { 1SS13E13R01 }\end{array}$ & $\begin{array}{r}\text { 1SS15EOSA01 } \\
\star 15 S 1 S E 0 S D 01 \\
15 S 1 S E 06001 \\
1 S S 1 S E 07 R 01\end{array}$ \\
\hline $\begin{array}{l}\text { 11S12E32R01 } \\
11 S 13 E 09001 \\
11 S 13 E 31005 \\
11 S 13 E 31 K 0 S\end{array}$ & $\begin{array}{r}12 S 14 E 33001 \\
\star 13 S 11 E 02 K 01 \\
\star 13 S 11 E 02 K 02 \\
\star 13 S 11 E 12 D 01\end{array}$ & $\begin{array}{l}13 S 14 E 18 R 01 \\
13 S 14 E 20 N 01 \\
13 S 14 E 21 N 06 \\
13 S 14 E 2 S J 04\end{array}$ & $\begin{array}{l}14 S 14 E 13 A 02 \\
14 S 14 E 14 \mathrm{NO} 1 \\
14 S 14 \mathrm{E} 15 \mathrm{AO} 1 \\
14 \mathrm{~S} 14 \mathrm{E} 15001\end{array}$ & $\begin{array}{l}\text { 1SS13E20001 } \\
1 S S 13 E 32 \mathrm{~F} 01 \\
1 \mathrm{SS} 14 \mathrm{E} 01002 \\
15514 \mathrm{E} 01 \mathrm{NO} 3\end{array}$ & $\begin{array}{r}\text { 1SS1SE08001 } \\
\star 1 \text { IS } 1 \text { SE10A01 } \\
\star 1 S S 1 S E 10002 \\
\text { 1SS1SE10J01 }\end{array}$ \\
\hline $\begin{array}{r}11 \mathrm{~S} 13 E 31 K 06 \\
11 \mathrm{~S} 13 \mathrm{E} 33 \mathrm{R} 02 \\
11 \mathrm{~S} 13 \mathrm{E} 36 \mathrm{R} 01 \\
\star 12 \mathrm{~S} 10 \mathrm{E} 01 \mathrm{CO} 1\end{array}$ & $\begin{array}{r}\star 13 S 11 E 12002 \\
13 S 12 E 04 A 01 \\
13 S 12 E 0 S R 02 \\
13 S 12 E 08 J 01\end{array}$ & $\begin{array}{l}13 S 14 E 2 S N O 3 \\
13 S 14 E 27 D 02 \\
13 S 14 E 27 N 01 \\
13 S 14 E 27 R 01\end{array}$ & $\begin{array}{l}\text { 14S14E15N03 } \\
\text { 14S14E17N01 } \\
\text { 14S14E18A01 } \\
\text { 14S14E18R02 }\end{array}$ & $\begin{array}{l}\text { 1SS14E01R01 } \\
\text { 1SS14E03A01 } \\
\text { 15S14E04NO1 } \\
\text { 1SS14E04R01 }\end{array}$ & $\begin{array}{r}\text { *1SS1SE13N01 } \\
\text { *1SS15E13R01 } \\
\text { 1SS1SE14B01 } \\
\text { 1SS1SE14L01 }\end{array}$ \\
\hline 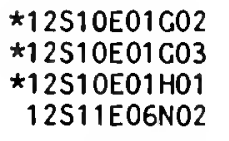 & $\begin{array}{r}* 13 S 12 E 17001 \\
13 S 12 E 17 N 02 \\
13 S 12 E 18 E 02 \\
13 S 12 E 18 \mathrm{CO} 1\end{array}$ & $\begin{array}{l}13 S 14 E 32001 \\
13 S 14 E 33001 \\
13 S 14 E 34 N 03 \\
13 S 14 E 34 R 01\end{array}$ & $\begin{array}{l}\text { 14S14E20N02 } \\
14 \text { S14E21D01 } \\
\text { 14S14E24N06 } \\
14 S 14 E 24 R 01\end{array}$ & $\begin{array}{l}\text { 1SS14E0SO01 } \\
15 S 14 E 06003 \\
1 S S 14 E 06 R 01 \\
15 S 14 E 11 D 03\end{array}$ & $\begin{array}{l}\text { 1SS1SE1SO01 } \\
\text { 1SS15E16R01 } \\
\text { 1SS1SE17A03 } \\
\text { ISS1SE18N03 }\end{array}$ \\
\hline $\begin{array}{r}\star 12 S 11 E 07 \mathrm{CO} 1 \\
\star 12 S 11 \mathrm{E} 07 \mathrm{G02} \\
12 S 11 \mathrm{E} 17 \mathrm{C01} \\
12 S 11 \mathrm{E} 17001\end{array}$ & $\begin{array}{r}\text { *13S12E18R01 } \\
13 S 12 E 20 F 02 \\
13 S 12 E 20 \mathrm{CO} 2 \\
13 S 12 \mathrm{E} 20 \mathrm{J01}\end{array}$ & $\begin{array}{l}\text { 13S1SE18M01 } \\
\text { 13S15E19L01 } \\
\text { 13S15E19M01 } \\
\text { 13S1SE19M02 }\end{array}$ & $\begin{array}{l}\text { 14S14E2SNO1 } \\
14 \text { S14E26D02 } \\
14 \text { S14E26N02 } \\
14514 E 27 N 02\end{array}$ & $\begin{array}{l}\text { 1SS14E12R01 } \\
15 S 14 E 13001 \\
1 S S 14 E 14002 \\
1 S S 14 E 16 A 01\end{array}$ & $\begin{array}{l}\text { 1SS1SE18R01 } \\
\text { 1SS1SE19NOS } \\
\text { 1SS1SE21001 } \\
\text { 1SS1SE21N03 }\end{array}$ \\
\hline
\end{tabular}


TABLE 10.--Wells with water levels indicative of the water table and dry wells which help define the water table--Continued

\begin{tabular}{|c|c|c|c|c|c|}
\hline $\begin{array}{r}\text { 1SS1SE22NO2 } \\
* 15 S 15 E 23 A 02 \\
15 S 15 E 23 D 02 \\
* 15 S 1 S E 24 B 01\end{array}$ & $\begin{array}{r}\text { 16S1SE09R01 } \\
\text { *16S1SE12N02 } \\
\text { 16S1SE13A02 } \\
\text { 16S1SE14001 }\end{array}$ & $\begin{array}{r}* 16 S 16 E 30 N 04 \\
* 16 S 16 E 32001 \\
* 16 S 16 E 32 \mathrm{NO} 2 \\
16 S 16 E 33 D 01\end{array}$ & $\begin{array}{r}17 \mathrm{~S} 16 \mathrm{E} 28 \mathrm{DO} 1 \\
17 \mathrm{~S} 16 \mathrm{E} 29 \mathrm{NO} 2 \\
* 17 \mathrm{~S} 16 \mathrm{E} 31 \mathrm{MO1} \\
* 17 \mathrm{~S} 16 \mathrm{E} 3 \mathrm{DO} 01\end{array}$ & $\begin{array}{r}\text { 18S16E03A01 } \\
* 18 S 16 E 04 D 01 \\
18 S 16 E 0 S E 01 \\
18 S 16 E 0 S R 02\end{array}$ & $\begin{array}{r}\text { 18S17E24N04 } \\
\text { 18S17E2SA01 } \\
\text { 18S17E35R02 } \\
\text { *18S18E02001 }\end{array}$ \\
\hline $\begin{array}{l}\text { 1SS1SE24N02 } \\
1 \text { SS1SE24R02 } \\
\text { 15S1SE26D02 } \\
\text { 15S1SE26R02 }\end{array}$ & $\begin{array}{r}\text { 16S1SE17N03 } \\
\text { 16S1SE18A01 } \\
\text { *16S15E18P01 } \\
\text { 16S1SE19H02 }\end{array}$ & $\begin{array}{l}16 \mathrm{~S} 16 \mathrm{E} 34 \mathrm{NO} 03 \\
16 \mathrm{~S} 16 \mathrm{E} 3 \mathrm{SA0} 1 \\
16 \mathrm{~S} 16 \mathrm{ESNN01} \\
16 \mathrm{~S} 16 \mathrm{E} 36 \mathrm{NO} 1\end{array}$ & $\begin{array}{l}\text { 17S16E33R03 } \\
17 \mathrm{~S} 17 \mathrm{E} 03 \mathrm{NO} 1 \\
17 \mathrm{~S} 17 \mathrm{E} 04 \mathrm{AO} 1 \\
17 \mathrm{~S} 17 \mathrm{E} 0 \mathrm{SN} 02\end{array}$ & $\begin{array}{l}\text { 18S16E06F01 } \\
18 \mathrm{~S} 16 \mathrm{E} 07 \mathrm{A0} 2 \\
18 \mathrm{~S} 16 \mathrm{E} 07 \mathrm{Q} 01 \\
18 \mathrm{~S} 16 \mathrm{E} 08 \mathrm{D} 02\end{array}$ & $\begin{array}{l}\text { 18S18E02R02 } \\
18 S 18 E 03 R 01 \\
18 S 18 E 04 N 01 \\
18 S 18 E 05 N 04\end{array}$ \\
\hline $\begin{array}{l}\text { 1SS1SE27N02 } \\
15 S 1 S E 27 R 01 \\
15 S 15 E 28 N 01 \\
15 S 15 E 29 N 03\end{array}$ & $\begin{array}{r}\text { *16S1SE20A01 } \\
\text { 16S1SE20E01 } \\
\text { 16S1SE20M01 } \\
\text { 16S1SE21A01 }\end{array}$ & $\begin{array}{r}* 16 \text { S17E16N01 } \\
\text { 16S17E19R01 } \\
* 16 S 17 E 20001 \\
* 16 S 17 E 21 A 01\end{array}$ & $\begin{array}{l}\text { 17S17E0SR01 } \\
17 \text { S17E06C01 } \\
17 \text { S17E06N02 } \\
17 S 17 E 07 N 01\end{array}$ & $\begin{array}{l}\text { 18S16E08M01 } \\
\text { 18S16E08M02 } \\
\text { 18S16E08N02 } \\
\text { 18S16E09N02 }\end{array}$ & $\begin{array}{l}\text { 18S18E07001 } \\
\text { 18S18E12R01 } \\
\text { 18S18E13E01 } \\
\text { 18S18E14D01 }\end{array}$ \\
\hline $\begin{array}{l}\text { 1SS1SE32N01 } \\
\text { 15S1SE33NO1 } \\
\text { 15S1SE33R01 } \\
\text { 15S1SE35N02 }\end{array}$ & $\begin{array}{r}\text { 16S1SE21N01 } \\
\text { 16S1SE21R01 } \\
\text { *16S1SE23D01 } \\
\text { *16S1SE24D01 }\end{array}$ & $\begin{array}{r}16 S 17 E 27 N 02 \\
16 S 17 E 28 N 03 \\
* 16 S 17 E 29 N 01 \\
* 17 S 14 E 02 D 01\end{array}$ & $\begin{array}{l}\text { 17S17E08N01 } \\
\text { 17S17E09N01 } \\
\text { 17S17E09R01 } \\
\text { 17S17E11N01 }\end{array}$ & $\begin{array}{l}18 \mathrm{~S} 16 \mathrm{E} 10001 \\
18 \mathrm{~S} 16 \mathrm{E} 10 \mathrm{~N} 01 \\
18 \mathrm{~S} 16 \mathrm{E} 12 \mathrm{NO} 1 \\
18 \mathrm{~S} 16 \mathrm{E} 12 \mathrm{R} 01\end{array}$ & $\begin{array}{l}\text { 18S18E14N01 } \\
18 \mathrm{~S} 18 \mathrm{E} 16 \mathrm{NO} 01 \\
18 \mathrm{~S} 18 \mathrm{E} 17 \mathrm{D} 01 \\
18 \mathrm{~S} 18 \mathrm{E} 17 \mathrm{~N} 01\end{array}$ \\
\hline $\begin{array}{l}\text { 1SS15E3SQ01 } \\
1 \text { SS1SE36Q01 } \\
\text { 1SS16E05E02 } \\
\text { 1SS16E13J01 }\end{array}$ & $\begin{array}{r}\star 16 S 1 S E 24 N 04 \\
\star 16 S 1 S E 27 A 01 \\
\star 16 S 1 S E 28 M 02 \\
16 S 1 S E 29 B 01\end{array}$ & $\begin{array}{r}* 17 S 14 E 14 A 02 \\
* 17 S 1 \text { SE02E01 } \\
17 S 15 E 06 P 02 \\
17 S 1 S E 07001\end{array}$ & $\begin{array}{l}\text { 17S17E11R02 } \\
\text { 17S17E1SR01 } \\
\text { 17S17E16R01 } \\
\text { 17S17E20A01 }\end{array}$ & $\begin{array}{l}\text { 18S16E1SR02 } \\
\text { 18S16E16R01 } \\
\text { 18S16E17001 } \\
\text { 18S16E17F01 }\end{array}$ & $\begin{array}{r}* 18 \mathrm{~S} 18 \mathrm{E} 18001 \\
* 18 \mathrm{~S} 18 \mathrm{E} 18 \mathrm{~N} 02 \\
18 \mathrm{~S} 18 \mathrm{E} 20 \mathrm{NO} 1 \\
18 \mathrm{~S} 18 \mathrm{E} 21 \mathrm{NO} 2\end{array}$ \\
\hline $\begin{array}{r}\text { 15S16E26A01 } \\
\text { 15S16E30R01 } \\
\text { 15S16E31N04 } \\
* 1 S S 16 E 32 R 02\end{array}$ & $\begin{array}{r}* 16 S 1 S E 29 F 01 \\
\text { *16S1SE32A04 } \\
16 S 15 E 33 K 01 \\
\text { 16S1SE33L01 }\end{array}$ & $\begin{array}{l}\text { *17S1SE11B01 } \\
\text { *17S1SE11C01 } \\
* 17 \text { S ISE13N04 } \\
\text { *17S1SE13N06 }\end{array}$ & $\begin{array}{l}\text { 17S17E21P01 } \\
17 \mathrm{~S} 17 \mathrm{E} 21 \mathrm{R} 02 \\
17 \mathrm{~S} 17 \mathrm{E} 23 \mathrm{HO} 1 \\
17 \mathrm{~S} 17 \mathrm{E} 24001\end{array}$ & $\begin{array}{r}\text { *18S16E17N03 } \\
\text { 18S16E17001 } \\
\text { 18S16E20A01 } \\
* 18 S 16 E 20 E 02\end{array}$ & $\begin{array}{l}\text { 18S18E21R01 } \\
18 \mathrm{~S} 18 \mathrm{E} 22 \mathrm{DO} 3 \\
18 \mathrm{~S} 18 \mathrm{E} 23 \mathrm{~N} 02 \\
18 \mathrm{~S} 18 \mathrm{E} 24002\end{array}$ \\
\hline $\begin{array}{l}\text { 15S16E33001 } \\
\text { 16S14E01A01 } \\
\text { 16S14E01R01 } \\
\text { 16S14E02A01 }\end{array}$ & $\begin{array}{r}\text { 16S16E01R01 } \\
* 16 S 16 E 02 N 01 \\
16 S 16 E 03 E 01 \\
* 16 S 16 E 03 N 01\end{array}$ & $\begin{array}{r}\text { *17S1SE14A02 } \\
\text { 17S1SE17R01 } \\
\text { *17S1SE24P02 } \\
\text { *17S1SE2SJ01 }\end{array}$ & $\begin{array}{l}\text { 17S17E25A01 } \\
\text { 17S17E26A01 } \\
\text { 17S17E27A01 } \\
\text { 17S17E27N01 }\end{array}$ & $\begin{array}{l}\text { 18S16E2OHO1 } \\
18 S 16 E 20 R 02 \\
18 S 16 E 22 \text { N03 } \\
\text { 18S16E23A03 }\end{array}$ & $\begin{array}{l}\text { 18S18E24N02 } \\
18 \mathrm{~S} 18 \mathrm{~B} 2 \mathrm{SNO} 1 \\
18 \mathrm{~S} 18 \mathrm{E} 26 \mathrm{NO} 03 \\
18 \mathrm{~S} 18 \mathrm{E} 27 \mathrm{NO} 4\end{array}$ \\
\hline $\begin{array}{l}\text { 16S14E02N02 } \\
\text { 16S14E02R01 } \\
16 \mathrm{~S} 14 \mathrm{E} 03 \mathrm{E} 02 \\
16 \mathrm{~S} 14 \mathrm{E} 03 \mathrm{NO} 1\end{array}$ & $\begin{array}{r}\text { *16S16E03N02 } \\
\text { 16S16E0SR03 } \\
\text { 16S16E10N02 } \\
\text { *16S16E12R01 }\end{array}$ & $\begin{array}{l}\text { 17S16E02NO2 } \\
17 \mathrm{~S} 16 \mathrm{E} 02 \mathrm{RO} 1 \\
17 \mathrm{~S} 16 \mathrm{E} 04 \mathrm{DO} 1 \\
17 \mathrm{~S} 16 \mathrm{E} 04 \mathrm{NO} 3\end{array}$ & $\begin{array}{l}\text { 17S17E27R04 } \\
\text { 17S17E28R03 } \\
17 \mathrm{~S} 17 \mathrm{E} 29 \mathrm{N03} \\
17 \mathrm{~S} 17 \mathrm{E} 30 \mathrm{A01}\end{array}$ & $\begin{array}{r}* 18 S 16 \mathrm{E} 24 \mathrm{A01} \\
\text { 18S16E28M01 } \\
\text { 18S16E33A06 } \\
* 18 \mathrm{~S} 16 \mathrm{E} 3 \mathrm{GO} 1\end{array}$ & $\begin{array}{l}\text { 18S18E30N02 } \\
\text { 18S18E32E02 } \\
\text { 18S18E32R01 } \\
\text { 18S18E34N01 }\end{array}$ \\
\hline $\begin{array}{r}16 S 14 \text { E04NO2 } \\
* 16 S 14 \text { EOSCO2 } \\
* 16 S 14 \text { E0SD01 } \\
16 S 14 E 09 A 02\end{array}$ & $\begin{array}{r}\text { 16S16E13D01 } \\
* 16 S 16 E 13 R 01 \\
\text { 16S16E14N02 } \\
* 16 S 16 E 15 A 02\end{array}$ & $\begin{array}{r}\text { *17S16E06R01 } \\
\text { 17S16E09A01 } \\
\text { 17S16E09R03 } \\
\text { 17S16E11N04 }\end{array}$ & $\begin{array}{l}\text { 17S17E31N02 } \\
17 \mathrm{~S} 17 \mathrm{E} 3 \mathrm{R} 01 \\
17 \mathrm{~S} 17 \mathrm{E} 34 \mathrm{R} 01 \\
17 \mathrm{~S} 17 \mathrm{E} 35 \mathrm{AO} 1\end{array}$ & $\begin{array}{r}\text { 18S16E34H01 } \\
\star 18 S 16 E 35 J 01 \\
\text { 18S17E01N01 } \\
\text { 18S17E03N03 }\end{array}$ & $\begin{array}{l}18 S 18 E 35 N 0 S \\
18 S 18 E 36 N 05 \\
18 S 18 E 36 R 01 \\
18 S 19 E 16 N 02\end{array}$ \\
\hline $\begin{array}{l}\text { 16S14E09H02 } \\
\text { 16S14E09NO1 } \\
\text { 16S14E10J01 } \\
\text { 16S14E10K02 }\end{array}$ & $\begin{array}{l}\text { 16S16E15N03 } \\
16 \mathrm{~S} 16 \mathrm{E} 17 \mathrm{A0} 1 \\
16 \mathrm{~S} 16 \mathrm{E} 17 \mathrm{D01} \\
16 \mathrm{~S} 16 \mathrm{E} 19 \mathrm{A0} 1\end{array}$ & $\begin{array}{l}17 \mathrm{~S} 16 \mathrm{E} 12 \mathrm{NO} 2 \\
17 \mathrm{~S} 16 \mathrm{E} 13 \mathrm{NO} 3 \\
17 \mathrm{~S} 16 \mathrm{E} 14 \mathrm{NO} 1 \\
17 \mathrm{~S} 16 \mathrm{E} 16 \mathrm{D} 01\end{array}$ & $\begin{array}{r}17 S 17 E 36 N 01 \\
* 17 S 17 E 36 R 01 \\
* 17 S 18 E 19 A 01 \\
17 S 18 E 26 N 01\end{array}$ & $\begin{array}{l}\text { 18S17E0SR01 } \\
\text { 18S17E06N01 } \\
\text { 18S17E07A01 } \\
\text { 18S17E07R01 }\end{array}$ & $\begin{array}{l}\text { 18S19E17D01 } \\
\text { 18S19E19C01 } \\
\text { 18S19E19R01 } \\
\text { 18S19E32H01 }\end{array}$ \\
\hline $\begin{array}{r}\text { 16S14E11R01 } \\
\star 16 \mathrm{~S} 14 \mathrm{E} 12 \mathrm{NO} 01 \\
* 16 \mathrm{~S} 14 \mathrm{E} 13 \mathrm{~F} 01 \\
\text { 16S14E13G02 }\end{array}$ & $\begin{array}{r}\text { *16S16E19Q01 } \\
\text { 16S16E20N02 } \\
\text { 16S16E21001 } \\
\text { 16S16E21N01 }\end{array}$ & $\begin{array}{r}\text { 17S16E16N02 } \\
\text { 17S16E16R01 } \\
* 17 \text { S16E17D02 } \\
\text { 17S16E20N01 }\end{array}$ & $\begin{array}{r}17 \mathrm{~S} 18 \mathrm{E} 28 \mathrm{NO} 2 \\
17 \mathrm{~S} 18 \mathrm{E} 28 \mathrm{R} 02 \\
* 17 \mathrm{~S} 18 \mathrm{E} 29 \mathrm{DO} 1 \\
17 \mathrm{~S} 18 \mathrm{E} 30 \mathrm{NO} 1\end{array}$ & $\begin{array}{r}\text { 18S17E09N02 } \\
\text { 18S17E10N01 } \\
\text { 18S17E11P01 } \\
\text { *18S17E13N03 }\end{array}$ & $\begin{array}{l}\text { 18S19E33J02 } \\
\text { 18S19E35J01 } \\
\text { 18S20E09M01 } \\
\text { 18S20E10M01 }\end{array}$ \\
\hline $\begin{array}{r}\text { 16S1SE01N01 } \\
\text { *16S1SE01R01 } \\
\text { 16S1SE02N04 } \\
\text { 16S1SE04N01 }\end{array}$ & $\begin{array}{r}\text { 16S16E22N01 } \\
* 16 S 16 E 23 A 01 \\
\text { 16S16E23N02 } \\
\text { 16S16E23R01 }\end{array}$ & $\begin{array}{l}\text { 17S16E21R01 } \\
\text { 17S16E22001 } \\
\text { 17S16E23R01 } \\
\text { 17S16E24R02 }\end{array}$ & $\begin{array}{r}17 S 18 E 30 R 01 \\
* 17 S 18 E 31 \mathrm{NO} \\
* 17 \mathrm{~S} 18 \mathrm{E} 32 \mathrm{NO} 1 \\
17 \mathrm{~S} 18 \mathrm{E} 3 \mathrm{~N} 02\end{array}$ & $\begin{array}{r}\text { *18S17E14A01 } \\
\text { 18S17E14N03 } \\
\text { 18S17E1SN0S } \\
\text { 18S17E16N01 }\end{array}$ & $\begin{array}{r}\text { 18S2OE19N01 } \\
* 19 S 16 E 01 \mathrm{AO} 2 \\
\star 19 \mathrm{~S} 16 \mathrm{E} 0 \mathrm{HH} 01 \\
* 19 \mathrm{~S} 16 \mathrm{ESH} 02\end{array}$ \\
\hline $\begin{array}{l}\text { 16S1SE04R01 } \\
16 S 1 S E 0 S N 01 \\
16 S 1 S E 07 N 02 \\
16 S 15 E 09 N 02\end{array}$ & $\begin{array}{r}\text { 16S16E24R01 } \\
\text { 16S16E27N01 } \\
\text { 16S16E27R01 } \\
\text { *16S16E30D01 }\end{array}$ & $\begin{array}{l}\text { 17S16E2SR01 } \\
\text { 17S16E26N03 } \\
\text { 17S16E26R01 } \\
\text { 17S16E27N02 }\end{array}$ & $\begin{array}{r}17 \mathrm{~S} 18 \mathrm{BE} 3 \mathrm{R} 01 \\
* 18 \mathrm{~S} 1 \mathrm{SE} 04 \mathrm{NO} 2 \\
18 \mathrm{~S} 16 \mathrm{E} 01 \mathrm{~N} 03 \\
18 \mathrm{~S} 16 \mathrm{E} 02 \mathrm{A01}\end{array}$ & $\begin{array}{r}\text { 18S17E20D01 } \\
\text { 18S17E21N01 } \\
\text { *18S17E21R01 } \\
\text { 18S17E22R01 }\end{array}$ & $\begin{array}{l}\text { *19S16E0SH03 } \\
\star 19 S 16 E 0 S P 01 \\
\star 19 S 16 E 17 C 02 \\
\star 19 S 16 E 20 B 01\end{array}$ \\
\hline
\end{tabular}


TABLE 10.--Wells with water levels indicative of the water table and dry wells which help define the water table--Continued

\begin{tabular}{|c|c|c|c|c|c|}
\hline $\begin{array}{l}\star 19 S 16 E 29 B 01 \\
\star 19 S 16 E 32 A 01 \\
\star 19 S 16 E 32 R 01 \\
\star 19 S 17 E 06 K 01\end{array}$ & $\begin{array}{l}\text { 19S19E09A01 } \\
\text { 19S19E10NO1 } \\
\text { 19S19E11B01 } \\
\text { 19S19E11J01 }\end{array}$ & $\begin{array}{r}\star 20 S 16 E 09 L 01 \\
20 S 16 E 10 H 03 \\
\star 20 S 16 E 1 S M 01 \\
\star 20 S 16 E 1 S N 01\end{array}$ & $\begin{array}{r}\text { 20S18E34R01 } \\
20 S 18 E 3 S 003 \\
20 S 18 E 3 S 001 \\
* 20 S 18 E 36004\end{array}$ & $\begin{array}{r}21 \text { S17E06N01 } \\
* 21 \text { S18E01A01 } \\
* 21 \text { S18E02N01 } \\
* 21 S 18 E 06 J 01\end{array}$ & $\begin{array}{r}* 21 S 19 E 041102 \\
21 S 19 E 06 N 01 \\
21 S 19 E 06 R 02 \\
21 S 19 E 07 R 01\end{array}$ \\
\hline $\begin{array}{l}\text { *19S17E08D02 } \\
\star 19 S 17 E 1 S N 02 \\
\star 19 S 17 E 16 B 01 \\
\star 19 S 17 E 16 C 01\end{array}$ & $\begin{array}{r}\text { 19S19E16N04 } \\
\text { 19S19E17A01 } \\
\text { *19S19E18N04 } \\
\text { 19S19E19A01 }\end{array}$ & $\begin{array}{l}\star 20 S 16 E 22 K 01 \\
\star 20 S 16 E 22001 \\
\star 20 S 16 E 26 L 01 \\
* 20 S 16 E 3 S H 01\end{array}$ & $\begin{array}{l}20 S 19 E 03001 \\
20 S 19 E 0 S N 01 \\
20 S 19 E 08 A 01 \\
20 S 19 E 11 A 03\end{array}$ & $\begin{array}{r}\star 21 S 18 E 07 C 01 \\
\star 21 S 18 E 10 D 03 \\
21 S 18 E 10 R 01 \\
21 S 18 E 12003\end{array}$ & $\begin{array}{l}\text { 21S19EO9NO1 } \\
\text { 21S19E10NO1 } \\
\text { 21S19E11001 } \\
\text { 21S19E17N01 }\end{array}$ \\
\hline $\begin{array}{r}\star 19 S 17 E 22 \mathrm{HO} 1 \\
\star 19 S 17 \mathrm{E} 22 \mathrm{HO} 2 \\
\star 19 S 17 \mathrm{E} 23001 \\
19 S 17 \mathrm{E} 30 \mathrm{AO} 2\end{array}$ & $\begin{array}{l}\text { 19S19E19001 } \\
\text { 19S19E20N01 } \\
\text { 19S19E21A01 } \\
\text { 19S19E21NO2 }\end{array}$ & $\begin{array}{r}\text { 20S17E11NO3 } \\
\text { 20S17E32R01 } \\
\star 20 S 18 E 01 N 01 \\
\text { 20S18E01R01 }\end{array}$ & $\begin{array}{l}\text { 20S19E11NO2 } \\
20 S 19 E 1 S H 01 \\
20 S 19 E 16 A 01 \\
20 S 19 E 16002\end{array}$ & $\begin{array}{l}21 \mathrm{~S} 18 \mathrm{E} 12 \mathrm{~N} 01 \\
21 \mathrm{~S} 18 \mathrm{E} 13 \mathrm{~A} 01 \\
21 \mathrm{~S} 18 \mathrm{E} 13 \mathrm{~N} 01 \\
21 \mathrm{~S} 18 \mathrm{E} 1 \mathrm{SR} 01\end{array}$ & $\begin{array}{l}\text { 21S19E1 8N02 } \\
\text { 21S19E21NO1 } \\
21 \text { S19E30NO1 } \\
21 \text { S19E31001 }\end{array}$ \\
\hline $\begin{array}{r}* 19 \mathrm{~S} 17 \mathrm{E} 36 \mathrm{HO} 2 \\
19 \mathrm{~S} 18 \mathrm{E} 02 \mathrm{NO} 1 \\
19518 \mathrm{E} 02 \mathrm{RO} 1 \\
* 19 \mathrm{~S} 18 \mathrm{E} 04 \mathrm{NO} 1\end{array}$ & $\begin{array}{l}\text { 19S19E23A01 } \\
\text { 19S19E26H03 } \\
\text { 19S19E26003 } \\
\text { 19S19E27D02 }\end{array}$ & $\begin{array}{l}\star 20 S 18 E 02 A 01 \\
\star 20 S 18 E 06003 \\
\star 20 S 18 E 07 B 01 \\
\star 20 S 18 E 07001\end{array}$ & $\begin{array}{l}\text { 20S19E17001 } \\
20 S 19 E 18 R 01 \\
20 S 19 E 20001 \\
20 S 19 E 21 A 01\end{array}$ & $\begin{array}{l}\star 21 \mathrm{~S} 18 \mathrm{E} 18 \mathrm{GO} 2 \\
\star 21 \mathrm{~S} 18 \mathrm{E} 18 \mathrm{JO2} \\
\star 21 \mathrm{~S} 18 \mathrm{E} 20 \mathrm{JO} 1 \\
\star 21 \mathrm{~S} 18 \mathrm{E} 20 \mathrm{KO} 1\end{array}$ & $\begin{array}{r}22 \mathrm{~S} 18 \mathrm{E} 01 \mathrm{~B} 01 \\
\star 22 \mathrm{~S} 18 \mathrm{E} 01002 \\
\star 22 \mathrm{~S} 18 \mathrm{E} 01 \mathrm{P} 01 \\
\star 22 \mathrm{~S} 18 \mathrm{E} 02 \mathrm{E} 01\end{array}$ \\
\hline $\begin{array}{r}\text { *19S18E0SNO2 } \\
\text { 19S18E10D01 } \\
* 19 \mathrm{~S} 18 \mathrm{E} 10 \mathrm{NO} 2 \\
\text { *19S18E12NO1 }\end{array}$ & $\begin{array}{l}\text { 19S19E30N01 } \\
\text { 19S19E31N02 } \\
\text { 19S19E31R01 } \\
\text { 19S19E32D01 }\end{array}$ & $\begin{array}{l}\star 20 S 18 E 07 R 01 \\
\star 20 S 18 E 11 \mathrm{NO} 2 \\
\star 20 S 18 E 13001 \\
\star 20 S 18 E 13 \mathrm{NO} 1\end{array}$ & $\begin{array}{l}\text { 2OS19E23N01 } \\
20 S 19 E 2 S D 02 \\
20 S 19 E 28 A 01 \\
20 S 19 E 29 D 01\end{array}$ & $\begin{array}{r}\star 21 S 18 E 21 R 01 \\
\star 21 S 18 E 22001 \\
21 S 18 E 24 N 03 \\
\star 21518 E 27 N 03\end{array}$ & $\begin{array}{l}* 22 \mathrm{~S} 18 \mathrm{E} 11 \mathrm{~B} 01 \\
* 22 \mathrm{~S} 18 \mathrm{E} 11 \mathrm{BO2} \\
* 22 \mathrm{~S} 18 \mathrm{E} 12 \mathrm{PO} \\
* 22 \mathrm{~S} 18 \mathrm{E} 12 \mathrm{R} 02\end{array}$ \\
\hline $\begin{array}{l}\text { 19S18E14R01 } \\
\text { 19S18E24001 } \\
\text { 19S18E33M02 } \\
\text { 19S19E02N01 } \\
\text { 19S19E07N01 }\end{array}$ & $\begin{array}{r}\text { 19S19E33D01 } \\
19 S 19 E 34002 \\
19 S 19 E 35 R 02 \\
\star 20 S 16 E 09001 \\
* 20 S 16 E 09002\end{array}$ & $\begin{array}{r}\star 20 S 18 E 13 R 02 \\
\star 20 S 18 E 18 R 01 \\
20 S 18 E 24 R 01 \\
\star 20 S 18 E 30 J 02 \\
20 S 18 E 34 N 01\end{array}$ & $\begin{array}{r}\text { *20S19E31001 } \\
\text { 20S19E32R01 } \\
20 S 19 E 33 A 01 \\
20 S 19 E 33 R 01 \\
20 S 19 E 3 S B 01\end{array}$ & $\begin{array}{l}\star 21 S 18 E 28 B 01 \\
\star 21 S 18 E 28 B 02 \\
\star 21 S 18 E 34 A 01 \\
\star 21 S 18 E 34 K 02 \\
\star 21 S 19 E 02 \mathrm{CO} 1\end{array}$ & $\begin{array}{l}\star 22 S 18 E 13 F 01 \\
\star 22 S 18 E 13 K 01\end{array}$ \\
\hline
\end{tabular}

TABLE 11.--Wells perforated below the water table and above the Corcoran Clay Member of the Tulare Formation

\begin{tabular}{lllll}
\hline 1OS1OE31D02 & $13 S 12 E 20002$ & $1 S S 14 E 30 C 01$ & $16 S 14 E 04 P 01$ & $16 S 16 E 0 S R 02$ \\
$11 S 10 E 36 L 01$ & $13 S 12 E 20 R 02$ & $1 S S 14 E 31 B 01$ & $16 S 14 E 13 G 01$ & $18 S 16 E 20 R 01$ \\
$12 S 12 E 03 R 02$ & $13 S 12 E 28002$ & $1 S S 14 E 32 N 03$ & $16 S 14 E 13 G 04$ & $18 S 16 E 23 A 01$ \\
$12 S 12 E 2 S R 02$ & $13 S 14 E 17 N 02$ & $1 S S 1 S E 09003$ & $16 S 15 E 18 P 02$ & $18 S 16 E 23 A 02$ \\
$12 S 13 E 07 N 01$ & $14 S 1 S E 08 C 04$ & $1 S S 1 S E 18 N 01$ & $16 S 1 S E 18 P 03$ & $18 S 16 E 33 A 02$ \\
$12 S 13 E 19 K 01$ & $14 S 1 S E 28 L 04$ & $1 S S 1 S E 18 N 02$ & $16 S 1 S E 19 H 01$ & $18 S 18 E 05 N 04$ \\
$13 S 12 E 17 N 01$ & $1 S S 13 E 11 B 02$ & $16 S 14 E 04 N 03$ & $16 S 15 E 32 A 03$ & $18 S 16 E 33 A 04$ \\
$13 S 12 E 18 E 01$ & $1 S S 13 E 12 N 04$ & $16 S 14 E 04 N 04$ & $16 S 16 E 03 E 02$ & $18 S 16 E 33 A 05$ \\
\hline
\end{tabular}




\section{SUMMARY}

A large number of wells have been drilled in the San Joaquin Valley to supply water for agriculture and to monitor water levels. Information from 5,860 of these wells was collected from several sources and compiled into a common data base. These wells were then organized into categories by comparing the location of the perforated lengths with the location of the hydrogeologic units. These categories were used to characterize the nature and distribution of the wells and to compile information on the groundwater flow system. In addition, a computerized system for storing and retrieving well information was developed.

Because of the varying degree of detail and quality of the data, only 2.547 wells had sufficient information for classification into four categories based on the hydrogeology: 1,114 wells are perforated in the semiconfined zone at depths less than or equal to 50 feet. 490 wells are perforated in the semiconfined zone at depths greater than 50 feet, 533 wells are perforated in the semiconfined and confined zolles, and 410 wells are perforated only in the confined zone. Additionally, wells perforated in the semiconfined zone at depths greater than 50 feet were classified by the type of deposits they were perforated in (Coast Range alluvium or Sierran sand).

Each hydrogeologically based category is represented by a map showing the location of wells in the category and by a list of those wells. The maps illustrate the areal distribution of the wells in a category. The lists allow the user to obtain more information from the data base with the use of the programs provided. This information can be used as a starting point for further investigations in the San Joaquin Valley.
Wells perforated in the semiconfined zone at depths less than or equal to 20 feet are evenly distributed over the area less than 20 feet from water. They generally are used as observation wells to monitor the shallow water table. The 50-foot wells are used to monitor water levels near the California Aqueduct.

Most wells perforated in the semiconfined zone at depths greater than 50 feet are perforated in the Sierran sand. A few wells are perforated in the Coast Range alluvium, but the coverage is sparse. The concentration of wells perforated in the Sierran sand indicates that there is a greater tendency to use Sierran sand than the Coast Range alluvium as a source of water. Moreover. a large percentage of the wells perforated in the Coast Range alluvium are part of the California Aqueduct monitoring system and are not production wells.

Most of the wells perforated in the semiconfined and confined zones are upslope of the valley trough in areas where the Sierran sand is absent. These wells have an average perforated length of 987 feet below the Corcoran and an average perforated length of 132 feet above the Corcoran. Wells in or near the valley trough where the Sierran sand is present have a shorter average perforated length below the Corcoran (555 feet) and a slightly longer average perforated length above the Corcoran (162 feet). This indicates that wells which are able to access the Sierran sand do not need to be drilled deep into the confined zone to obtain an adequate yield of water.

Similarly, wells perforated only in the confined zone are predominantly upslope of the valley trough. These wells also reflect the influence of the presence or absence of Sierran sand 
above the Corcoran. Wells in the valley trough have an average perforated length of 628 feet, and wells upslope of the valley trough have a longer average perforated length of 987 feet.

Wells perforated only in the confined zone also are concentrated near the creeks. This indicates a tendency for locating wells in the coarser grained sediments of the upper-fan areas, rather than in the finer grained sediments of the interfan areas.

\section{REFERENCES CITED}

Belitz, Kenneth, 1988, Character and evolution of the ground-water flow system in the central part of the western San Joaquin Valley, California: U.S. Geological Survey OpenFile Report 87-573, 34 p.

Bull, W.B., 1975, Land subsidence due to ground-water withdrawal in the Los Banos-Kettleman City area, California, Part 2. Subsidence and compaction of deposits: U.S. Ceological Survey Professional Paper 437-F, $90 \mathrm{p}$.

Bull, W.B., and Miller, R.E., 1975, Land subsidence due to groundwater withdrawal in the Los BanosKettleman City area, California, Part 1. Changes in the hydrologic environment conducive to subsidence: U.S. Geological Survey Professional Paper 437-E, $71 \mathrm{p}$.
Gilliom, R.J., and others, 1989, Preliminary assessment of sources, distribution and mobility of selenium in the San Joaquin Valley. California: U.S. Geological Survey WaterResources Investigations Report 88-4186, $129 \mathrm{p}$.

Ireland, R.L., 1963, Description of wells in the Los Banos-Kettleman City area, Merced, Fresno, and Kings Counties, California: U.S. Geological Survey Open-File Report, 519 p. Laudon, Julie, and Belitz, Kenneth, 1989, Texture and depositional history of near-surface alluvial deposits in the central part of the western San Joaquin Valley California: U.S. Geological Survey Open-File Report 89-235, $19 \mathrm{p}$.

LeBlanc, R.A., 1970, Data for wells in the Dos Palos-Kettleman City area, San Joaquin Valley, California: U.S. Geological Survey Open-File Report, $72 \mathrm{p}$.

Miller, R.E., Green, J.H., and Davis, G.H., 1971, Geology of the compacting deposits in the Los BanosKettleman City subsidence area, California: U.S. Ceological Survey Professional Paper 497-E, $46 \mathrm{p}$.

Page, R.W., 1986, Geology of the fresh ground-water basin of the Central Valley, California, with texture maps and sections: U.S. Geological Survey Professional Paper 1401-C, $54 \mathrm{p}$. 


\section{APPENDIX--SAMPLE DATA AND PROGRAM DOCUMENTATION}

The following Fortran programs were designed to retrieve well records from the water-level data base. Data Recovery, which is a compilation of many of the smaller programs described later, is an interactive program which allows the user to query the data base for specific information. Data Recovery accepts as input any file that is in a "standard format." Methods of recovering data from the input file include: (1) interactive entry of specific well locations; (2) a list-driven search using an external file containing well locations; (3) recovering wells in a specified township/range; and (4) a search of the entire input file. Searches can be further restricted by imposing ranges or limits on the dates of record and/or the depth of the wells.

Strip Headers accepts any subset of the water-level data base as input, and creates a listing of only the headers without the water-level entries. The list of headers has many uses, including its role as input to Depth_Sort, which searches through the list of headers and produces well records for the subset of wells having depths within a specified range. Strip Loc also uses the output from Strip Headers, and converts it to a list of well locations (township, range, section, quarter-quarter section, and well number) which can be used as input for the list-driven search in Data_Recovery.

Date Select accepts any subset of the water-level data base as input, and retrieves header and water-level information for these wells that have a measurement on a user-specified date. The output consists of one line per successful search, containing the location, water level (on the specified date), and depth of the well. WL Diff compares two files created by Date Select, and calculates the differences between the water levels. 
Sample Data (in "standard" USBR format):

1234567890123456789012345678901234567890123456

$\begin{array}{llll}0005 M 13 S 13 E 13 D 0110 & 6 & N 001683 & 0 \\ 830110 & 020400203000880007801952 & 0 & \\ 830405 & 020400203000780006801962 & 0 & \\ 830706 & 020400203000700006001970 & 0 & \\ 831004 & 020400203000810007101959 & 0 & \\ 840117 & 020400203000860007601954 & 0 & \\ 840402 & 020400203000820007201958 & 0 & \\ 840702 & 020400203000620005201978 & 0 & \\ 841003 & 020400203000860007601954 & 0 & \\ 850102 & 020400203000870007701953 & 0 & \\ 850403 & 020400203000730006301967 & 0 & \\ 850708 & 020400203000650005501975 & 1 & \end{array}$

The first line, called a "header", is organized as follows:

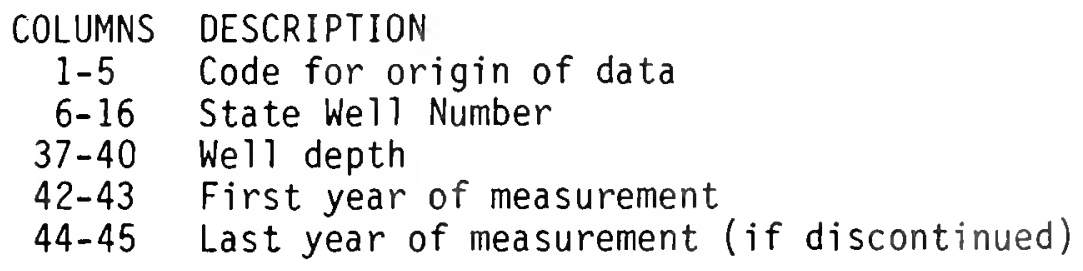

The remaining lines are organized as follows:

COLUMNS DESCRIPTION

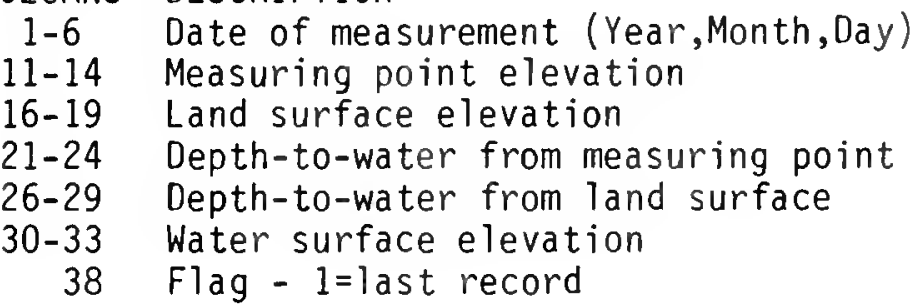

Note: There are implied decimals between the 13th and 14th, 18th and 19th, 23rd and 24 th, 28 th and 29 th, and 32 nd and 33 rd columns.

If the digit after the implied decimal is not a number, then the value is negative. 


\section{PROGRAM DATA_RECOVERY}

C S.P.PHILLIPS $05 / 87$ This program is an interactive data retrieval system $C$ designed to accept data in "standard" USBR format, and output that data which meets the user-specified requirements for LOCATION, DEPTH, and DATE.

INPUT :

Example Input (in "standard" USBR format):

$$
123
$$

1234567890123456789012345678901234567890123456

Q005M13S13E13D0110 6 N001683 0

$830110 \quad 020400203000880007801952 \quad 0$

$830405 \quad 020400203000780006801962 \quad 0$

$830706 \quad 020400203000700006001970 \quad 0$

$831004020400203000810007101959 \quad 0$

$840117020400203000860007601954 \quad 0$

$840402020400203000820007201958 \quad 0$

$840702020400203000620005201978 \quad 0$

$841003020400203000860007601954 \quad 0$

$850102020400203000870007701953 \quad 0$

$850403 \quad 020400203000730006301967 \quad 0$

$850708020400203000650005501975 \quad 1$

The first line, called a "header", is organized as follows:

COLUMNS DESCRIPTION

1-5 Code for origin of data

6-16 State Well Number

37-40 Well depth

42-43 First year of measurement

44-45 Last year of measurement (if discontinued)

The remaining lines are organized as follows:

COLUMNS DESCRIPTION

1-6 Date of measurement (Year, Month, Day)

11-14 Measuring point elevation

16-19 Land surface elevation

21-24 Depth-to-water from measuring point

26-29 Depth-to-water from land surface

30-33 Water surface elevation

$38 \quad$ Flag $-1=$ last record

OUTPUT:

The output consists of well records that satisfy the user-specified requirements, which are defined in two phases. The first phase involves the geographic bounds of the search, and offers the following options:

1) Interactively enter specific well locations.

2) Use list of well locations contained in an external file.

3) Search a specified township/range.

4) Let the data in the input file determine the geographic bounds. 
$C$ The second phase allows the user to select additional criteria for a C successful search. One or more of the following options may be applied C to any of the geographic searches chosen in the first phase:

1) Search for well within a specified depth range.

2) Search for well records within a specified date range.

3) Search for well records with a particular date.

The default output is a file containing sequential well records for those wells satisfying the search criteria. The user has the option of creating a separate file for each well.

$C \star \star \star \star$ Begin MAIN program COMMON /OFILES/ OFILE,BDFILE

COMMON /INFILS/ INFILE

COMMON /FLAGS/ PASSFL,SAVEFL, SEPFLG, SORTFL

COMMON /HDRS/ HEADER

COMMON /CHRS/ HLDATE,MONTHS, YEAR, YEARMO

COMMON / INTG/ DEEP, NUMMON(15), NUMYR, SHALL

C Global variables

CHARACTER HEADER $\star 80$, HLDATE $\star 4$, MONTHS $* 120$, SEPFLG $\star 1, Y$ YEAR $* 30$,

$+$ YEARMO * 30

CHARACTER PASSFL $\star 1$, SAVEFL $\star 1, S O R T F L \star 1$

INTEGER BDFILE, DEEP, SHALL, INF ILE, NUMMON, NUMYR, OF ILE

C Local variables

CHARACTER 1 OPTION $(5)$

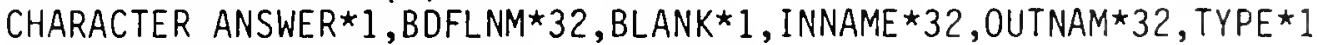

C

INTEGER MINDEX, MON,OPTNUM, TMON, YINDEX

C Initialize variables

INFILE $=30$

OFILE $=31$

$B D F I L E=5$

BLANK $=1$

C Initialize/reset variables

10 CONTINUE

$D E E P=0$

SHALL $=0$

HLDATE $=1 \quad 1$

$\operatorname{YEAR}(1: 2)=1$

$\operatorname{YEARMO}(1: 2)=1 \quad 1$

OPTNUM $=0$

NUMYR $=0$

TMON $=0$

SEPFLG $={ }^{\prime} N^{\prime}$ 
C Ask series of questions to define user needs, and open appropriate files WRITE $(1,300)$ BLANK

WRITE $(1,300)$ 'How do you wish to recover well data?'

WRITE $(1,300)$ BLANK

WRITE $(1,300)$ ' 1) Enter well location(s) from the terminal'

WRITE $(1,300)$ ' 2) Read well locations from a file'

WRITE $(1,300)$ ' 3) Gather data from specified Township/Range'

WRITE $(1,300)$ ' 4) Gather data from entire input file'

WRITE $(1,300)$ BLANK

WRITE $(1,300)$ 'ENTER 1, 2, 3, or 4:'

READ $(1,300)$ TYPE

WRITE $(1,300)$ BLANK

WRITE $(1,300)$ 'Additional options:'

20 CONTINUE

OPTNUM=OPTNUM+1

WRITE $(1,300)$ BLANK

WRITE $(1,300)$ ' 1 ) Specify depth range'

$\operatorname{WRITE}(1,300)$ ' 2) Specify date range'

WRITE $(1,300)$ ' 3) Specify particular date - Year(s) only'

WRITE $(1,300)$ ' 4) Specify particular date-Year(s)+Month(s)'

$\operatorname{WRITE}(1,300)$ ' 5) None'

WRITE $(1,300)$ BLANK

$\operatorname{WRITE}(1,300)$ 'ENTER 1, 2, 3, 4, or 5:'

$\operatorname{READ}(1,300)$ OPTION(OPTNUM)

IF(OPTION (OPTNUM).NE. ' 5') THEN

WRITE $(1,300)$ 'Would you like to use another option $(Y / N)$ ?'

READ $(1,300)$ ANSWER

IF(ANSWER.EQ.' 'Y') GOTO 20

ENDIF

WRITE $(1,300)$ 'Enter the name of the INPUT file containing'

WRITE $(1,300)$ '"standard" raw data'

$\operatorname{READ}(1,300)$ INNAME

OPEN (INFILE, FILE = INNAME)

WRITE $(1,300)$ 'Is the above input file sorted by location $(Y / N)$ ?'

$\operatorname{READ}(1,300)$ SORTFL

IF(SORTFL.EQ.' 'Y') THEN

IF(TYPE.EQ.'1') THEN

WRITE $(1,300)^{\prime}$ 'Is your list of well locations sorted $(Y / N)$ ?' READ $(1,300)$ SORTFL

ELSE IF(TYPE.EQ.' '2') THEN

WRITE $(1,300)$ 'Is the file containing locations sorted $(Y / N)$ ?' READ ( 1,300$)$ SORTFL

ELSE IF(TYPE.EQ.' '3') THEN WRITE $(1,300)$ 'Is your list of Township/Ranges sorted by' WRITE $(1,300)$ 'Township and Range, respectively $(Y / N)$ ?'

$\operatorname{READ}(1,300)$ SORTFL

ENDIF 
ENDIF

WRITE $(1,300)$ 'Enter the name of the OUTPUT file:'

READ $(1,300)$ OUTNAM

OPEN (OFILE, FILE=OUTNAM)

WRITE $(1,300)$ 'You have the option to create a separate file for'

WRITE $(1,300)$ 'each set of well data that passes the search'

WRITE $(1,300)$ 'criteria - would you like this option $(Y / N)$ ?'

$\operatorname{READ}(1,300)$ ANSWER

IF (ANSWER.EQ. ' $Y$ ') SEPFLG $=$ ' $Y$ '

WRITE $(1,300)$ 'Would you like to save a file of headers/locations'

$\operatorname{WRITE}(1,300)$ 'that do not pass the search criteria $(Y / N)$ ?'

$\operatorname{READ}(1,300)$ ANSWER

IF(ANSWER.EQ.' ' $Y$ ') THEN

SAVEFL $=' Y$ '

WRITE $(1,300)$ 'Enter file name: '

$\operatorname{READ}(1,300)$ BDFLNM

OPEN (BDFILE, FILE=BDFLNM)

C

ENDIF

If options are chosen, ask user for additional information

DO $30 \quad I=I, O P T N U M$

WRITE $(1,300)$ BLANK

IF(OPTION(I).EQ. 'I') THEN

WRITE $(1,300)$ 'Specify a depth range -'

WRITE $(1,300)$ ' Enter the largest well depth desired:'

$\operatorname{READ}(I, 200)$ DEEP

WRITE $(1,300)$ ' Enter the smallest well depth desired:'

$\operatorname{READ}(1,200)$ SHALL

ELSE IF(OPTION(I).EQ.' '2') THEN

WRITE $(1,300)$ 'Specify a date range -'

WRITE $(1,300)$ ' Enter largest year (last 2 digits only):'

READ ( I ,300) HLDATE $(3: 4)$

$\operatorname{WRITE}(1,300)$ ' Enter smallest year (last 2 digits only):'

$\operatorname{READ}(1,300)$ HLDATE $(1: 2)$

ELSE IF(OPTION(I).EQ.' '3') THEN

WRITE $(1,300)$ 'Specify particular $\operatorname{Year}(\mathrm{s})$-'

40

CONTINUE

NUMYR $=$ NUMYR +1

$Y$ INDEX $=($ NUMYR $* 2)-1$

WRITE $(1,300)$ ' Enter a desired year (last 2 digits only): '

$\operatorname{READ}(1,300)$ YEAR(YINDEX:(YINDEX+I))

WRITE $(I, 300)$ 'Are more years desired $(Y / N)$ ?'

$\operatorname{READ}(1,300)$ ANSWER

IF(ANSWER.EQ.' $\gamma$ ') GOTO 40

ELSE IF(OPTION(I).EQ. '4') THEN

50

WRITE(I,300) 'Specify particular Year(s) and Month(s) -'

CONTINUE

NUMYR $=$ NUMYR +1

$Y$ INDEX $=($ NUMYR $* 2)-1$

WRITE $(1,300)$ ' Enter a desired year (last 2 digits only):'

$\operatorname{READ}(1,300)$ YEARMO (YINDEX: (YINDEX+1))

$\mathrm{MON}=0$ 
60

CONTINUE

$M O N=M O N+1$

$T M O N=T M O N+1$

MINDEX $=($ TMON*2) -1

WRITE $(1,301)$ ' Enter one desired month for the year 19',

$+$

$\operatorname{WRITE}(1,300)$ ' (2 DIGITS - leading 0 is often required): '

$\operatorname{READ}(1,300)$ MONTHS (MINDEX:(MINDEX+1))

$\operatorname{WRITE}(1,300)$ 'Are there more months for this year $(Y / N)$ ?'

$\operatorname{READ}(1,300)$ ANSWER

IF (ANSWER.EQ.' 'Y') GOTO 60

NUMMON $($ NUMYR $)=$ MON

$\operatorname{WRITE}(1,300)$ 'Do you wish to enter another year $(\mathrm{Y} / \mathrm{N})$ ?'

$\operatorname{READ}(1,300)$ ANSWER

IF(ANSWER.EQ.' 'Y') GOTO 50

\section{ENDIF}

30 CONTINUE

C

Go to appropriate subroutine where header/well locations will be

selected/entered, and sent to SEARCH.

IF (TYPE.EQ.' '1') CALL INTERACT

IF (TYPE.EQ.' ''') CALL LOCFILE

IF (TYPE.EQ. ' $3^{\prime}$ ) CALL TRSEARCH

IF(TYPE.EQ. '4') CALL ALLSEARCH

ENDFILE (OFILE)

CLOSE (INFILE)

CLOSE (OFILE)

C

WRITE $(1,300)$ 'Would you like to continue recovering data $(Y / N)$ ?'

$\operatorname{READ}(1,300)$ ANSWER

IF(ANSWER.EQ.' $Y$ ') GOTO 10

200 FORMAT( I 4 )

300 FORMAT (A)

301 FORMAT (2A)

STOP

END

$C \star \star \star \star$ End MAIN program

$\mathrm{C}$

C

C

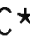

SUBROUTINE SEARCH

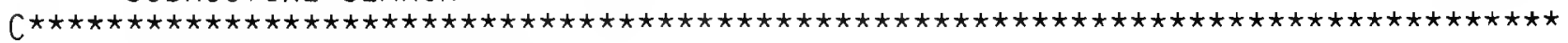

COMMON /OFILES/ OFILE,BDFILE

COMMON / INFILS/ INFILE

COMMON /FLAGS/ PASSFL, SAVEFL, SEPFLG, SORTFL

COMMON / INTG/ DEEP, NUMMON(15), NUMYR, SHALL

COMMON /HDRS/ HEADER

COMMON /CHRS/ HLDATE, MONTHS, YEAR, YEARMO 
C Global variables

CHARACTER HEADER $* 80, H L D A T E \star 4, M O N T H S * 120, S E P F L{ }^{\star} 1, Y$ YEAR
YEARMO 30,30

CHARACTER PASSFL 1 , SAVEFL $\star 1$, SORTFL $* 1$

C Local variables

CHARACTER FILNAM* 12 , LINE*80, DATRNG*1, DEPRNG* 1 , PASS* 1 , SPYEAR* 1 ,

$+$

INTEGER DEPTH, FILNUM, MINDEX, WRI TES, YINDEX

C

Initialize variables

DATRNG $=$ ' $Y$ '

DEPRNG $=$ ' $Y$ '

SPYEAR $=$ ' $Y$ '

SPYMON $=$ ' $Y$ '

PASSFL $=$ ' $P$ '

$\operatorname{FILNAM}(1: 1)={ }^{\prime} L$ '

FILNUM $=33$

$c$

C Determine needs

IF (DEEP.EQ.0) DEPRNG $=$ 'N'

IF (HLDATE.EQ.' ') DATRNG $=$ ' $N$ '

$\operatorname{IF}(\operatorname{YEAR}(1: 2) \cdot E Q . '$ ' $) \operatorname{SPYEAR}={ }^{\prime} N$ '

C

IF (YEARMO $(1: 2) . E Q . '$ ') SPYMON $=$ ' $N$ '

C First check to see if header meets specs - if yes, write it out - if no,

$C$ advance to the last line of record and RETURN

IF (DEPRNG.EQ. ' $Y$ ') THEN

READ (HEADER, 200) DEPTH

IF ((DEPTH.LT.SHALL).OR. (DEPTH.GT.DEEP)) GOTO 90

ENDIF

IF (DATRNG.EQ.' 'Y') THEN

IF(HEADER $(44: 45) . E Q . '$ ') THEN

IF (HEADER(42:43).GT.HLDATE(1:2)) GOTO 90

ELSE IF( (HEADER (42:43).GT.HLDATE $(1: 2))$.OR.

$+\quad$ (HEADER(44:45).LT.HLDATE(3:4))) THEN

GOTO 90

ENDIF

ENDIF

$c$

$C$ If this point is reached, the header meets specs, so write out to file WRITE(OFILE,300) HEADER

C

C

Search for and write out data that meets specs WRITES $=0$

10 READ(INFILE, 300) LINE

PASS $={ }^{\prime} N{ }^{\prime}$

TMON $=0$

IF(SPYEAR.EQ.'Y') THEN

DO $20 \mathrm{I}=1$, NUMYR

YINDEX $=(I * 2)-1$

$\operatorname{IF}(\operatorname{LINE}(1: 2)$.EQ.YEAR(YINDEX:(YINDEX +1$)))$ GOTO 50 
ELSE IF(SPYMON.EQ. 'Y') THEN

DO $30 \quad I=1$, NUMYR

$Y$ INDEX $=(1 * 2)-1$

IF (LINE $(I: 2)$. EQ.YEARMO(YINDEX:(YINDEX+1))) THEN

DO $40 \mathrm{~L}=\mathrm{I}$, NUMMON( I)

TMON $=$ TMON +1

MINDEX $=(T M O N * 2)-I$

40

IF ( LINE( $3: 4)$.EQ.MONTHS(MINDEX:(MINDEX+I))) GOTO 50 CONTINUE

ELSE

TMON $=$ TMON+NUMMON (I)

\section{ENDIF}

30 CONTINUE

ELSE

PASS $={ }^{\prime} Y{ }^{\prime}$

ENDIF

C

GOTO 60

50 PASS $=$ ' $\gamma$ '

$C$ If the line of data passed inspection, write it out, and note that data

c was written to the output file. If no data was written for a given

C header (WRITES=0), the header, which was already written to the output

C file, must be erased (or written-over).

60 IF(PASS.EQ. 'Y') THEN

WRITES =WRITES + I

IF( (WRITES.EQ.I).AND.(SEPFLG.EQ.'Y')) THEN

$\operatorname{FILNAM}(2: 12)=\operatorname{HEADER}(6: 16)$

OPEN(FILNUM, FILE =FILNAM)

WRITE(FILNUM, 300) HEADER

ENDIF

IF(SEPFLG.EQ. 'Y') WRITE(FILNUM, 300) LINE

WRITE(OFILE, 300) LINE

C

ENDIF

C Check for end of record

IF ( LINE(38:38).EQ.' I') GOTO 80

GOTO 10

C

C

Check to see if any data was written out - if not, set it up so that the header will be overwritten. If data was written out, set the flag on the last entry to "I", indicating the final line of data for the preceeding header. 
80 IF(WRITES.EQ.0) THEN

BACKSPACE OFILE

$P A S S F L=' F '$

ELSE

BACKSPACE OFILE

READ(OFILE, 300) LINE

$\operatorname{LINE}(38: 38)=11$ '

BACKSPACE OFILE

WRITE(OFILE, 300) LINE

IF(SEPFLG.EQ. ' $Y$ ') CLOSE (FILNUM)

ENDIF

C

GOT0 99

C If header failed, read up to next header, and RETURN

90 READ (INFILE, 250) FLAG

IF(FLAG.NE.' '1') GOTO 90

PASSFL $=$ ' $F$ '

C

200 FORMAT $(36 X, 14)$

$250 \operatorname{FORMAT}(37 X, A 1)$

300 FORMAT $(A)$

99 RETURN

END

C

C

$$
C \star
$$

SUBROUTINE HDRSEARCH (LOCATN)

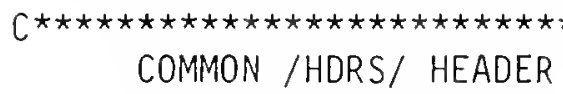

COMMON / INFILS/ INFILE

COMMON /FLAGS/ PASSFL, SAVEFL, SEPFLG, SORTFL

C Global variables

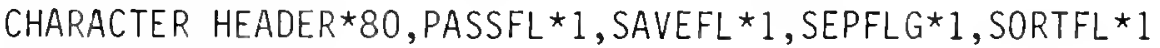

INTEGER INFILE

C Local variables

CHARACTER LOCATN* 11, LINE*80, FLAG*1

C

IF(SORTFL.EQ.'N') REWIND INFILE

PASSFL $=' P$ '

C

10 READ(INFILE, 300, END $=80$ ) LINE

IF (LINE (6:16).EQ.LOCATN) THEN

HEADER $=$ LINE

GOTO 99

ELSE

20 READ(INFILE, 200) FLAG

IF(FLAG.NE. '1') GOTO 20 
ENDIF

GOTO 10

C

80 PASSFL $={ }^{\prime} F^{\prime}$

REWIND INFILE

C

200 FORMAT $(37 X, A 1)$

300 FORMAT(A)

99 RETURN

END

C

C

$c \star \star x+$

SUBROUTINE INTERACT

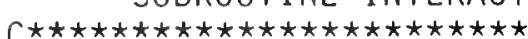

COMMON /OFILES/ OFILE,BDFILE

COMMON /FLAGS/ PASSFL, SAVEFL, SEPFLG, SORTFL

COMMON / HDRS/ HEADER

C Global variables

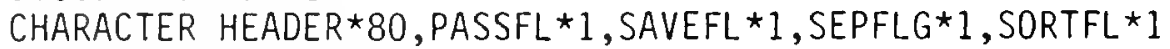

INTEGER BDFILE,OFILE

C Local variables

CHARACTER * 11 LOCATN (100)

CHARACTER BLANK^1

INTEGER NUMBER

C

BLANK $=1 \quad 1$

WRITE $(1,300)$ BLANK

WRITE $(1,300)$ 'WELL LOCATION entry section: '

WRITE $(1,300)$ BLANK

WRITE $(1,300)$ ' How many well locations would you like'

WRITE $(1,300)^{\prime}$ to enter $(\max 100)$ ?'

$\operatorname{READ}(1,200)$ NUMBER

WRITE $(1,300)$ BLANK

DO $10 \mathrm{I}=1$, NUMBER

WRITE $(1,250)^{\prime}$ Enter the 11 digit location for well number', I

READ (1,300) LOCATN(I)

10 CONTINUE

C Send each location to SEARCH, one at a time

DO $20 \quad I=1$, NUMBER

CALL HDRSEARCH (LOCATN(I))

IF (PASSFL.EQ. ' $\left.F^{\prime}\right)$ THEN

I F(SAVEFL.EQ.' ' $Y$ ') WRITE(BDFILE, 300) LOCATN(I)

ELSE

CALL SEARCH

IF (PASSFL.EQ.' $F$ ') THEN

IF(SAVEFL.EQ.' 'Y') WRITE(BDFILE, 300) LOCATN(I)

\section{ENDIF}

\section{ENDIF}

20 CONTINUE

C 


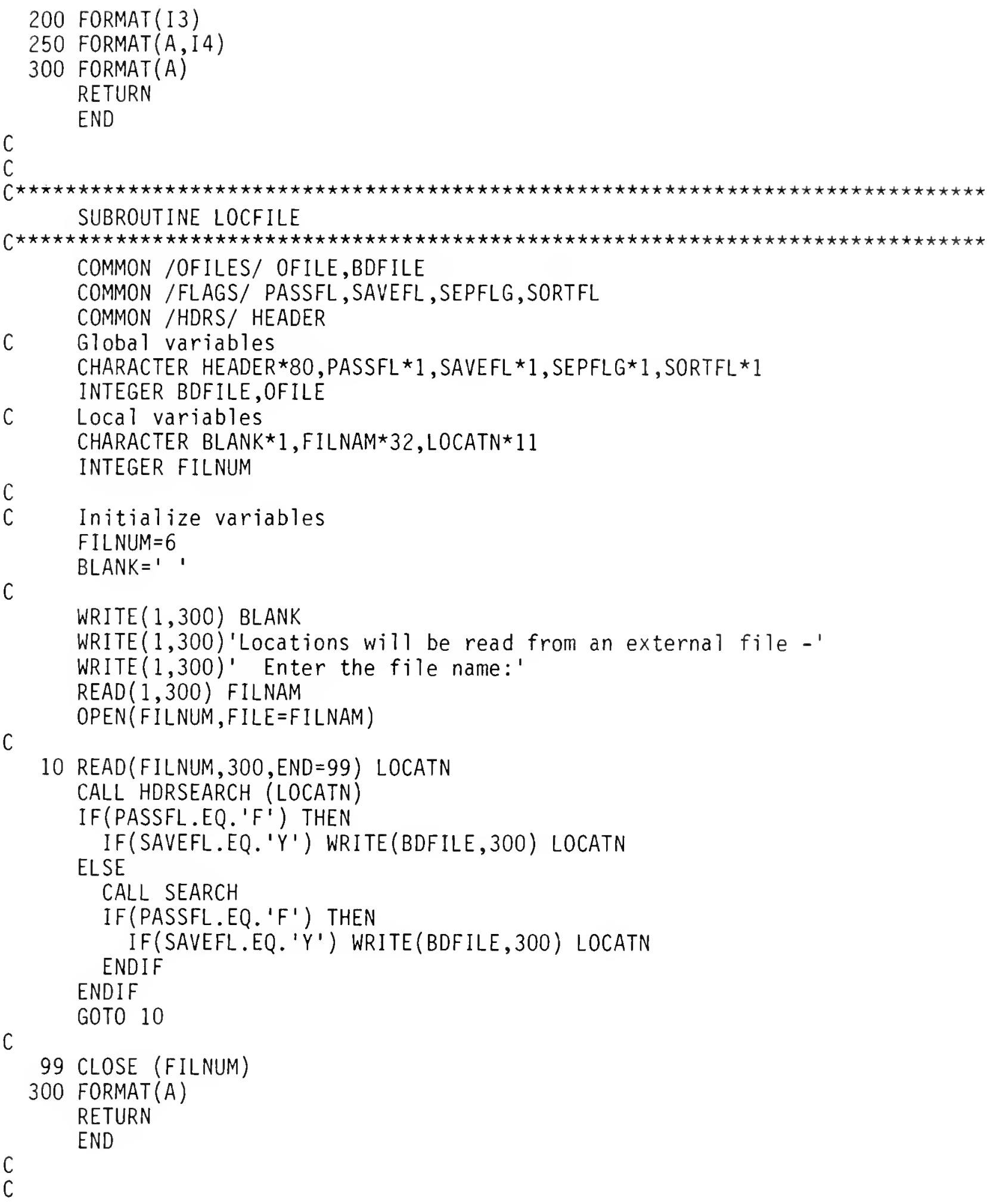




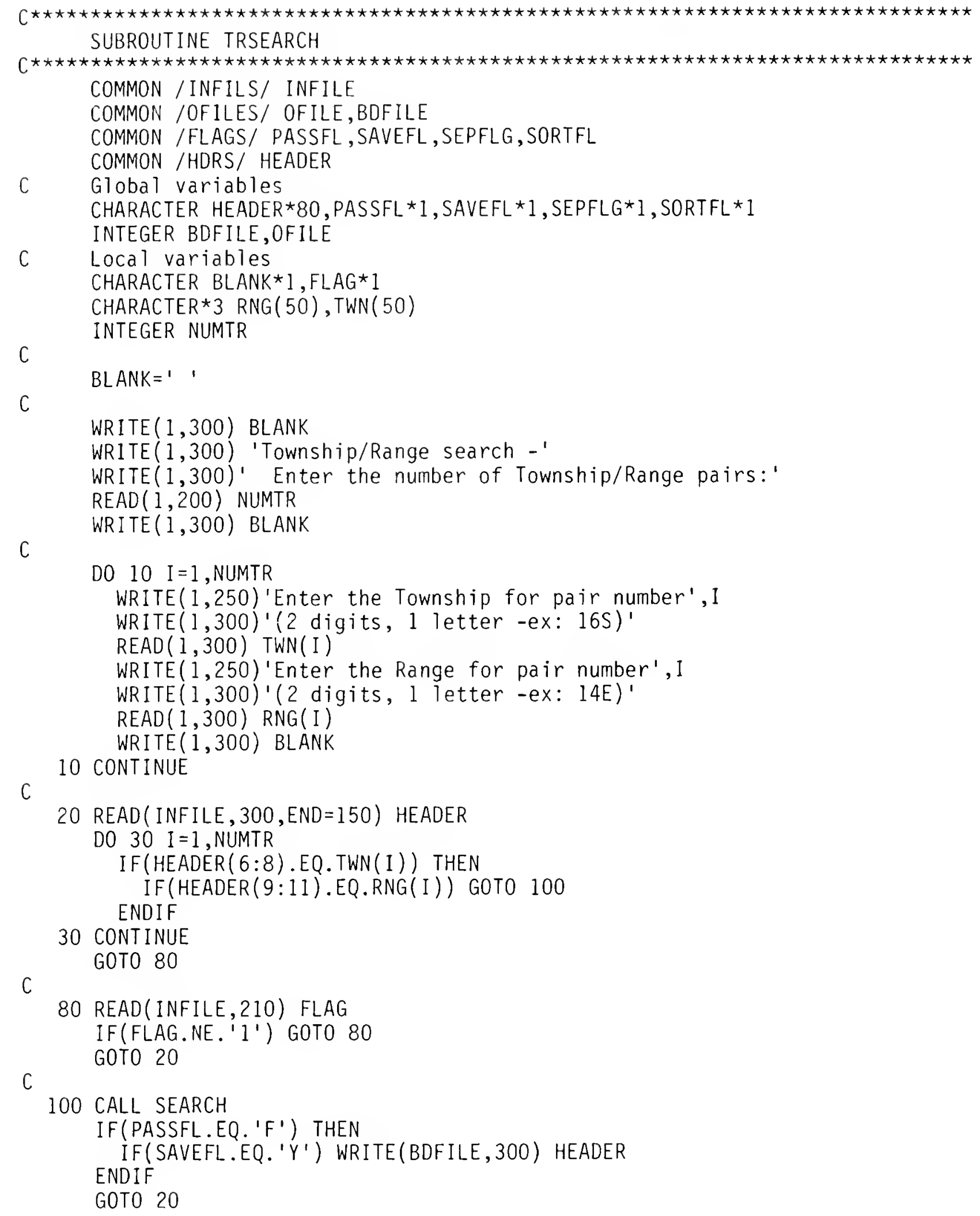


C

200 FORMAT(I2)

2 IO FORMAT $(37 X, A 1)$

250 FORMAT $(A, I 4)$

300 FORMAT $(A)$

150 RETURN

END

$\mathrm{C}$

$\mathrm{C}$

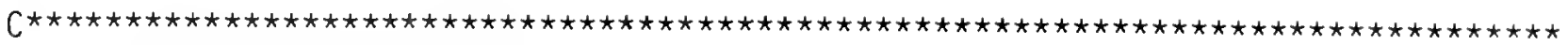
SUBROUTINE ALLSEARCH

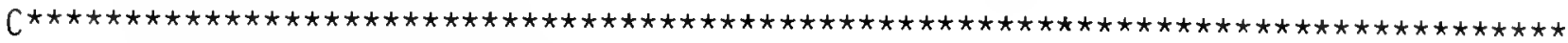

COMMON / INFILS/ INFILE

COMMON /OFILES/ OFILE,BDFILE

COMMON /FLAGS/ PASSFL, SAVEFL,SEPFLG,SORTFL

COMMON /HDRS/ HEADER

C Global variables

CHARACTER HEADER *80,PASSFL ${ }^{\star} 1$, SAVEFL * 1, SEPFLG*I, SORTFL ${ }^{\star} I$

C

INTEGER BDFILE,OFILE

10 READ(INFILE, 300,END=99) HEADER

CALL SEARCH

IF (PASSFL.EQ.' ' ${ }^{\prime}$ ) THEN

IF(SAVEFL.EQ.' 'Y') WRITE(BDFILE, 300) HEADER

ENDIF

GOTO 10

C

300 FORMAT(A)

99 RETURN

END 


\section{PROGRAM STRIP HEADERS}

C S.P.PHILLIPS $2 / 85$ This program creates a file of headers given well

C data in "standard" USBR format.

C

C INPUT:

C Example Input (in "standard" USBR format):

C

C

C Example Output (given example input):

C

C

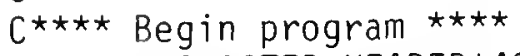

Q005M13S13E1300110 6 N0016 $83 \quad 0$

C CHARACTER HEADER $* 48$, IC $\star 1$, INFILE $* 32,0$ FILE*32

WRITE $(1,300)$ 'Enter INPUT file name (max 32 char): ' $\operatorname{REAO}(1,300)$ INFILE

WRITE $(1,300)$ 'Enter OUTPUT file name (max 32 char):'

$\operatorname{READ}(1,300)$ OFILE

OPEN $(30, F I L E=I N F I L E)$

$\operatorname{OPEN}(40, F I L E=O F I L E)$

C

$10 \operatorname{READ}(30,300, \mathrm{END}=99)$ HEADER

WRITE $(40,300)$ HEADER

$20 \operatorname{READ}\left(30, '(37 X, A 1)^{\prime}\right)$ IC

IF (IC.EQ. ' 0 ') GOTO 20

GOTO 10

C

300 FORMAT $(A)$

99 STOP

ENO 
PROGRAM DEPTH SORT

C S.P.PHILLIPS $7 / 86$ This program collects well data within a

C specified depth range given "standard" USBR format.

C

C

C

C

C

C

C

C

$c$

C

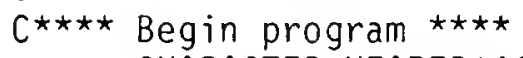

CHARACTER HEADER $* 46$, DATA $* 38$, INFILE $* 32$, OFILE 32

OUTPUT:

- Format is identical to input

C INTEGER DEPTH,MINDEP,MAXDEP

C Interactively determine user needs WRITE $(1,310)$

WRITE $(1,300)$ 'Enter name of INPUT file:'

$\operatorname{READ}(1,300)$ INFILE

$\operatorname{OPEN}(30, \mathrm{FILE}=$ INFILE)

WRITE $(1,300)$ 'Enter name of OUTPUT file:'

$\operatorname{READ}(1,300)$ OFILE

$\operatorname{OPEN}(40, F I L E=O F I L E)$

$\operatorname{WRITE}(1,300)$ 'Enter minimum depth (integer): '

$\operatorname{READ}(1,200)$ MINDEP

$\operatorname{WRITE}(1,300)$ 'Enter maximum depth (integer):'

C

$\operatorname{READ}(1,200)$ MAXDEP

10 CONTINUE

$\operatorname{READ}(30,300, E N D=99)$ HEADER

READ (HEADER, 210) DEPTH

IF ( (DEPTH.GE.MINDEP).AND. (DEPTH.LE.MAXDEP)) THEN

20 CONTINUE

$\operatorname{READ}(30,300)$ DATA

WRITE $(40,300)$ DATA

IF (DATA(38:).NE.'I') GOTO 20

ELSE

30

CONTINUE

$\operatorname{READ}(30,300)$ DATA

IF (DATA(38:).NE. 'I') GOTO 30

ENDIF

GOTO 10

200 FORMAT (I3)

210 FORMAT $(37 X, I 3)$

300 FORMAT (A)

310 FORMAT( )

99 STOP

END 
PROGRAM STRIP LOC

C S.P.PHILLIPS 05/87 This program reads in a list of "standard" USBR

$C$ headers, and returns only the location in the proper format for the file to be read by DATA_RECOVERY (for list-driven search).

INPUT:

Example of header in "standard" USBR format:

Q005M13S13E13D0110 6 N001683 0

C

C OUTPUT :

C Example output (given example input):

C

C

C

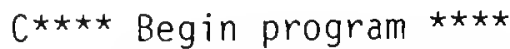

CHARACTER INFILE*32,LOC*11,OFILE*32

C

WRITE $(1,300)$ 'Enter INPUT file name (max 32 char):'

$\operatorname{READ}(1,300)$ INFILE

WRITE $(1,300)$ 'Enter OUTPUT file name ( $\max 32$ char):'

$\operatorname{READ}(1,300)$ OFILE

OPEN $(30, F I L E=I N F I L E)$

OPEN $(40, F I L E=O F I L E)$

C

$I=0$

10 CONTINUE

$\operatorname{READ}(30, '(5 X, A 11) ', E N D=99) \operatorname{LOC}$

$I=I+1$

$\operatorname{WRITE}(40,300)$ LOC

GOTO 10

C

$99 \operatorname{WRITE}(1,200)$ I

200 FORMAT (//, 'Total number of wells is:', I5)

$300 \operatorname{FORMAT}(A)$

STOP

END 


\section{PROGRAM DATE SELECT}

C S.P.PHILLIPS $12 / \overline{8} 5$ This program accepts well data in 'standard' USBR

$C$ format, and outputs the location, water level, and depth for those wells

$C$ with a water level measurement on the user-specified date.

C

C INPUT:

C Example Input (in "standard" USBR format):

C

$\mathrm{C}$

C

Q005M13S13E1300110

6 N0016 $83 \quad 0$

$830706 \quad 020400203000700006001970 \quad 0$

$840702020400203000620005201978 \quad 0$

$850708020400203000650005501975 \quad 1$

OUTPUT:

Example Output given the example input, and specifying the date $7 / 84$ :

Q005M13S13E13D01 $\quad 197.8 \quad 0016$

C

$C \star \star \star \star$ Begin MAIN program

CHARACTER ANSWER ${ }^{\star} 1$, DECIMAL $* 1, H E A D E R \star 80$, INFILE*32, LINE*38,

C

$+$

$M O N T H \star 2, O F I L E \star 32, Y E A R \star 2$

C

DECIMAL $={ }^{\prime} .1$

C User entry section (interactive)

WRITE $(1,300)$ 'Enter INPUT file name (max 32 chars):'

$\operatorname{READ}(1,300)$ INFILE

WRITE $(1,300)$ 'Enter OUTPUT file name (max 32 chrs):'

$\operatorname{READ}(1,300)$ OFILE

$\operatorname{OPEN}(30, F I L E=I N F I L E)$

$\operatorname{OPEN}(40, F I L E=0 F I L E)$

WRITE $(1,300)$ 'This program selects data given a specific date:'

$\operatorname{WRITE}(1,300)$ 'Would you like to include the MONTH $(Y / N)$ ?'

$\operatorname{READ}(1,300)$ ANSWER

IF(ANSWER.EQ. 'N') GOTO 5

$\operatorname{WRITE}(1,300)$ ' Enter the MONTH (2 digits): '

$\operatorname{READ}(1,300)$ MONTH

$\operatorname{WRITE}(1,300)$ ' Enter the YEAR (2 digits):'

$\operatorname{READ}(1,300)$ YEAR

GOTO 10

$5 \operatorname{WRITE}(1,300)$ ' Enter the YEAR (2 digits):'

$\operatorname{READ}(1,300)$ YEAR

C GOTO 30 
C This procedure is used if a particular month is specified $10 \operatorname{READ}(30,300, \operatorname{END}=99)$ HEADER I OUT $=1$

$20 \operatorname{READ}(30,300)$ LINE IF((LINE( $1: 2) \cdot$ EQ.YEAR).AND. (LINE( $3: 4) \cdot E Q \cdot M O N T H))$ THEN IF(IOUT.EQ. 1) WRITE $(40,250)$

$+\operatorname{HEADER}(1: 16), \operatorname{LiNE}(30: 32), \mathrm{DECIMAL}, \operatorname{LinE}(33: 34), \operatorname{HEADER}(37: 40)$ IOUT $=2$

ENDIF

IF(LINE(38:).NE. 'I') GOTO 20 GOTO 10

C

C

This procedure is used when only the year is specified $30 \operatorname{READ}(30,300, \mathrm{END}=99)$ HEADER

$40 \operatorname{READ}(30,300)$ LINE

IF(LINE $(1: 2)$.EQ.YEAR) WRITE $(40,250)$

$+\operatorname{HEADER}(1: 16), \operatorname{LINE}(30: 32), \mathrm{DECIMAL}, \operatorname{LINE}(33: 34)$

IF(LINE(38:).NE. ' 1 ') GOTO 40

GOTO 30

C

250 FORMAT $(A 16,5 X, A 3, A 1, A 2,5 X, A 4)$

300 FORMAT(A)

99 STOP

END 
PROGRAM WL DIFF

C S.P.PHILLIPS $02 / 87$ This program reads two files in the format

$C$ of the output from DATE SELECT, and produces a file containing

$C$ the locations common to both, and the difference between the

C water levels.

C

C INPUT:

C Example of input file 1:

$\mathrm{C}$

C

$\mathrm{C}$

C

$\mathrm{C}$

$\mathrm{C}$

C

C

C

C

C

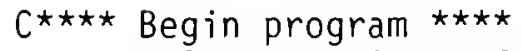

CHARACTER LOC $1 * 16$, LOC_ $2 * 16$, INFIL $1 * 32$, INF IL $2 * 32,0$ OFILE*32

INTEGER DEPTH $\overline{1}$, DEPTH2

C

$\operatorname{WRITE}(1,310)$

WRITE $(1,300)$ 'Enter name of Input file $1:{ }^{\prime}$

$\operatorname{READ}(1,300)$ INF IL 1

$\operatorname{OPEN}(30, \mathrm{FILE}=$ INFIL I $)$

WRITE $(1,300)$ 'Enter name of Input file $2:$ '

$\operatorname{READ}(1,300)$ INFIL2

$\operatorname{OPEN}(31, \mathrm{FILE}=\mathrm{INFIL2})$

WRITE $(1,300)$ 'Enter name of Output file:'

$\operatorname{READ}(1,300)$ OFILE

C

$\operatorname{OPEN}(40, \mathrm{FILE}=$ OFILE)

$\operatorname{READ}(30,200)$ LOC 1 ,WSELEV1,DEPTH1

$\operatorname{READ}(31,200)$ LOC $_{-}^{-}$, WSELEV2,DEPTH2

C

Check to see if locations are, or = to each other, and

deal with each case separately.

IF(LOC 1(6:16).GT.LOC_2(6:16)) THEN

READ(31, 200, END=99) LOC 2, WSELEV2, DEPTH2

ELSE IF(LOC $1(6: 16) . L T . L O \bar{C} 2(6: 16))$ THEN

\section{ELSE}

$\operatorname{READ}\left(30,20 \overline{0}, \mathrm{END}^{2} 99\right)$ LOC_ $\overline{1}$, WSELEV 1, DEPTH 1

DIFF $=$ WSELEV1-WSELEV2

WRITE $(40,200)$ LOC 1,DIFF, DEPTH1

$\operatorname{READ}(30,200, \mathrm{END}=9 \overline{9})$ LOC 1 , WSELEV 1, DEPTH 1

$\operatorname{READ}\left(31,200, \mathrm{END}^{2} 99\right) \quad \mathrm{LOC}_{-}^{-} 2$,WSELEV2,DEPTH2

ENDIF

GOTO 10

200 FORMAT $(A 16,5 X, F 5.1,5 X, 15)$

300 FORMAT (A)

310 FORMAT( )

99 STOP

END 








